\title{
The Asymptotic Safety Scenario in Quantum Gravity - An Introduction -
}

\author{
M. NiedermaieR* \\ Laboratoire de Mathematiques et Physique Theorique \\ CNRS/UMR 6083, Université de Tours \\ Parc de Grandmont, 37200 Tours, France
}

\begin{abstract}
The asymptotic safety scenario in quantum gravity is reviewed, according to which a renormalizable quantum theory of the gravitational field is feasible which reconciles asymptotically safe couplings with unitarity. All presently known evidence is surveyed: (a) from the $2+\epsilon$ expansion, (b) from the perturbation theory of higher derivative gravity theories and a 'large $N$ ' expansion in the number of matter fields, (c) from the 2-Killing vector reduction, and (d) from truncated flow equations for the effective average action. Special emphasis is given to the role of perturbation theory as a guide to 'asymptotic safety'. Further it is argued that as a consequence of the scenario the selfinteractions appear two-dimensional in the extreme ultraviolet. Two appendices discuss the distinct roles of the ultraviolet renormalization in perturbation theory and in the flow equation formalism.
\end{abstract}

${ }^{*}$ Membre du CNRS. 


\section{Contents}

1 Survey of the scenario and evidence for it $\quad 2$

1.1 Survey of the scenario . . . . . . . . . . . . . . . 2

1.2 Coarse graining and dynamically adjusted background data . . . . . . . . 15

1.3 Evidence for asymptotic safety . . . . . . . . . . . . . . . 19

1.4 Some working definitions . . . . . . . . . . . . . . . . . 30

1.5 Discussion of possible objections . . . . . . . . . . . . . . . . . . . . . 34

2 The role of perturbation theory $\quad 38$

2.1 Does Newton's constant run? . . . . . . . . . . . . . . . . 38

2.2 Is a non-Gaussian fixed point visible in PT? . . . . . . . . . . . . . . 41

2.3 Can unitarity and renormalizability be reconciled? . . . . . . . . . . . . 46

3 Dimensional reduction of residual interactions in UV 48

4 Conclusions $\quad 55$

$\begin{array}{ll}\text { A Renormalizing the nonrenormalizable } & 56\end{array}$

A.1 Perturbation theory and continuum limit . . . . . . . . . . . . 58

A.2 Functional flow equations and UV renormalization . . . . . . . . . . . . . 64 


\section{Survey of the scenario and evidence for it}

The quest for a physically viable theory of quantized gravitation is ongoing; in part because the physics it ought to describe is unknown, and in part because different approaches may not 'approach' the same physics. The most prominent contenders are string theory and loop quantum gravity, with ample literature available on either sides. For book-sized expositions see for example [68, 125, 132]. The present review surveys a circle of ideas which differ in several important ways from these approaches; we refer to [110] for a more detailed account with a slightly different emphasis.

\subsection{Survey of the scenario}

In brief, the scenario delineates conditions under which a functional integral based quantum theory of gravity can be viable beyond the level of an effective field theory: first a physics premise ("antiscreening") is made about the selfinteraction of the quantum degrees of freedom in the ultraviolet. Second, the effective diminution of the relevant degrees of freedom in the ultraviolet (on which morally speaking all approaches agree) is interpreted as universality in the statistical physics sense in the vicinity of an ultraviolet renormalization group fixed point. Third, the resulting picture of microscopic geometry is fractal-like with a local dimensionality of two.

The concrete implementation of these ideas has begun only recently and led to a number of surprising results to be reviewed here. Part of the physics intuition, on the other hand, dates back to an 1979 article by S. Weinberg [152], see also [66]. Motivated by the analogy to the asymptotic freedom property of nonabelian gauge theories, the term "asymptotic safety" was suggested in [152], indicating that physically motivated running couplings should be "safe" from divergencies at all scales. Following this suggestion we shall refer to the above circle of ideas as the "asymptotic safety scenario" for quantum gravity. For convenient orientation we display the main features in overview:

\section{Asymptotic safety scenario - main ideas:}

- The gravitational field itself is taken as the prime carrier of the relevant classical and quantum degrees of freedom; its macro- and micro-physics are related through a renormalization flow.

- As the basic physics premise stipulate that the physical degrees of freedom in the ultraviolet interact predominantly antiscreening.

- Based on this premise benign renormalization properties in the ultraviolet are plausible. The resulting "Quantum Gravidynamics" can then be viewed as a peculiar quasi-renormalizable field theory based on a non-Gaussian fixed point. 
- In the extreme ultraviolet the residual interactions appear two-dimensional.

The first point is shared by the effective field theory framework for quantum gravity, the others are needed to go beyond it in a functional integral based approach. The rationale for trying to so is twofold: first, the effective field framework gives rise only to very few universal corrections which are quantitatively too small to be phenomenologically interesting. Second, once the physics premise underlying a regularized functional integral for gravity has been made a "UV completion" based simply on removal of the regulator, if feasible, is physically well-motivated and computationally seamless.

A strategy centered around a functional integral picture was indeed adopted early on [101] but is now mostly abandoned. A functional integral over geometries of course has to differ in several crucial ways from one for fields on a fixed geometry. This lead to the development of several formulations (canonical, covariant [44, 45, 46], proper time $[142,143]$ and covariant Euclidean [74, 64]). As is well-known the functional integral picture is also beset from severe technical problems [140, 42, 64, 105]. Nevertheless this should not distract attention from the fact that a functional integral has a physics content which differs from the physics content of other approaches. For want of a better formulation we shall refer to this fact by saying that a functional integral picture "takes the degrees of freedom of the gravitational field seriously also in the quantum regime".

Let us briefly elaborate on that. Arguably the cleanest intuition to "what quantizing gravity might mean' comes from the functional integral picture. Transition or scattering amplitudes for nongravitational processes should be affected not only by one geometry solving the gravitational field equations, but by a 'weighted superposition' of 'nearby possible' off-shell geometries. The rationale behind this intuition is that all known (microscopic) matter is quantized that way, and using an off-shell matter configuration as the source of the Einstein field equations is in general inconsistent, unless the geometry is likewise off-shell. Moreover, relativistic quantum field theory suggests that the matter-geometry coupling is effected not only through averaged or large scale properties of matter. For example nonvanishing connected correlators of a matter energy momentum tensor should be a legitimate source of gravitational radiation as well, as should be the Casimir energy, see $[55,58]$. Of course this doesn't tell in which sense the geometry is off-shell, nor which class of possible geometries ought to be considered and be weighed with respect to which measure. Rapid decoherence, a counterpart of spontaneous symmetry breaking, and other unknown mechanisms may in addition mask the effects of the superposition principle. Nevertheless the argument suggests that the degrees of freedom of the gravitational field should be taken seriously also in the quantum regime, roughly along the lines of a functional integral.

Doing so one readily arrives at the effective field theory description of quantum gravity, see [33] for a recent review. It is a commonly accepted criterion that a theory of quantum gravity, even one evading a functional integral over geometries, should match whatever universal results are obtained from an effective field theory framework. The issue at stake thus is the extent to which different "UV completions" of the effective field theory 
description have a physics content different from the latter and from each other. Of course in the absence of empirical guidance the 'true' physics of quantum gravity is unknown; so for the time being it will be important to try to isolate differences in the physics content of the various "UV completions". By physics content we mean here qualitative or quantitative results for the values of "quantum gravity corrections" to generic physical quantities in the approach considered. Generic physical quantities should be such that they in principle capture the entire invariant content of a theory. In a conventional field theory S-matrix elements by and large have this property, in canonical general relativity Dirac observables play this role $[7,147,48]$. In quantum gravity, in contrast, no agreement has been reached on the nature of such generic physical quantities.

The present scenario proposes a UV completion proper, one which is based on the very same physics principles that make the effective field theory description so credible and which renders the crucial interpolating regime computationally accessible. We share the viewpoint expressed by Wilczek in [153]: "Whether the next big step will require a sharp break from the principles of quantum field theory, or, like the previous ones, a better appreciation of its potentialities, remains to be seen". Here we center the discussion around the above four main ideas, and, for short, call a quantum theory of gravity based on them Quantum Gravidynamics. For the remainder of Section 1.1 we now discuss a number of key issues that arise.

In any functional integral picture one has to face the crucial renormalizability problem. Throughout we shall be concerned exclusively with (non-)renormalizability in the ultraviolet. The perspective on the nature of the impasse entailed by the perturbative nonrenormalizability of the Einstein-Hilbert action (see Bern [21] for a review), however, has changed significantly since the time it was discovered by 't Hooft and Veltmann [140]. First, the effective field theory framework applied to quantum gravity provides unambiguous answers some lowest order corrections despite the perturbative nonrenormalizability of the 'fundamental' action, as stressed by Donoghue (see [27, 33] and references therein). The role of an a-priori microscopic action is moreover strongly deemphasized when a Kadanoff-Wilson view on renormalization is adopted. We shall give a quick reminder on this framework in Appendix A. Applied to gravity it means that the Einstein-Hilbert action should not be considered as the microscopic (high energy) action, rather the renormalization flow itself will dictate to a certain extent which microscopic action to use, and whether or not there is a useful description of the extreme ultraviolet regime in terms of 'fundamental' (perhaps nonmetric) degrees of freedom. The extent to which this is true hinges on the existence of a fixed point with a renormalized trajectory emanating from it. The fixed point guarantees universality in the statistical physics sense. If there is a fixed point any action on a renormalized trajectory describes identically the same physics on all energy scales lower than the one where it is defined. Following the trajectory back (almost) into the fixed point one can in principle extract unambiguous answers for physical quantities on all energy scales.

Compared to the effective field theory framework the main advantage of genuine renor- 
malizability lies not primarily in the gained energy range in which reliable computations can be made, but rather that one has a chance to properly identify 'large' quantum gravity effects at low energies (assuming they exist). The effective field theory framework rests on a decoupling assumption: there exists a potentially process-dependent scale $M_{\text {eff }}$ such that the low energy degrees of freedom $\left(E / M_{\text {eff }} \ll 1\right)$ relevant for the process obey an approximately autonomous dynamics. Based on this assumption some unambiguously defined low energy effects of quantized gravity can be identified, but are found to be suppressed by the powers of energy scale/Planck mass expected on dimensional grounds. However in the presence of massless degrees of freedom the decoupling assumption may fail (mediated e.g. by anomalies [10] or by spontaneous symmetry breaking) and the extent to which it is valid in 'quantized' gravity is a dynamical problem. In a theory of quantum gravidynamics this dynamical problem can be investigated: the effect of high energy (Planck scale) processes can in principle be computationally propagated through many orders of magnitudes down to accessible energies, where they may leave a detectable low energy imprint.

Note that the nature of the 'fundamental' degrees of freedom is of secondary importance in this context. From the viewpoint of renormalization theory it is the universality class that matters not the particular choice of dynamical variables. Once a functional integral picture has been adopted even nonlocally and nonlinearly related sets of fields or other variables may describe the same universality class - and hence the same physics.

Generally, the arena on which the renormalization group acts is a space of actions or, equivalently, a space of (regularized) measures. A typical action has the form: $\sum_{\alpha} u_{\alpha} P_{\alpha}$, where $P_{\alpha}$ are interaction monomials (including kinetic terms) and the $u_{\alpha}$ are scale dependent coefficients. The subset $u_{i}$ which cannot be removed by field redefinitions are called essential parameters, or couplings. Usually one makes them dimensionless by taking out a suitable power of the scale parameter $\mu, \mathrm{g}_{i}(\mu)=\mu^{-d_{i}} u_{i}(\mu)$. In the following the term essential coupling will always refer to these dimensionless variants. We also presuppose the principles according to which a (Wilson-Kadanoff) renormalization flow is defined on this arena. For the convenience of the reader a brief glossary is included Section 1.4. In the context of Quantum Gravidynamics some key notions (coarse graining operation, unstable manifold and continuum limit) have a somewhat different status which we outline below.

Initially all concepts in a Wilson-Kadanoff renormalization procedure refer to a choice of coarse graining operation. It is part of the physics premise of a functional integral approach that there is a physically relevant distinction between coarse grained and fine grained geometries. On a classical level this amounts to the distinction, for example, between a perfect fluid solution of the field equations and one generated by its $10^{30}$ or so molecular constituents. A sufficiently large set of Dirac observables would be able to discriminate two such spacetimes. One can also envisage a vacuum counterpart of this distinction and view the coarse graining scale as analogous to an 'intrinsic clock' variable in general relativity. Whenever we shall refer later on to "coarse grained" versus "fine grained" geometries we have a similar picture in mind for the ensembles of 
off-shell geometries entering a (regularized) functional integral. For example, the value of integrated curvature invariants like $\left(\nabla_{\alpha} R_{\beta \gamma \delta \rho} \nabla^{\alpha} R^{\beta \gamma \delta \rho}\right)^{2}$ may provide a rough measure for the coarseness. Tested proposals for an intrinsic coarse graining scale for geometries are however presently not available.

As a substitute one can define the coarse graining with respect to a state-dependent dynamically adjusted background metric. Let $\bar{g}_{\alpha \beta}$ be an initially prescribed background metric and suppose that it has been used to define a "background covariant" notion of coarse graining, e.g. by referring to the spectrum of a covariant differential operator built from $\bar{g}$. The coarse graining can then be used to construct the functional integral averages \langle\rangle$_{\bar{g}}$ subject to suitable boundary conditions that encode information about the state vector. Eventually one obtains a functional $\mathcal{O} \mapsto\langle\mathcal{O}\rangle_{F(\bar{g})}$ ("a state", roughly in the algebraic sense) which depends parameterically on the background $\bar{g}$ via a functional $F(\bar{g})$. In a second step one then selfconsistently adjusts the background metric to one solving

$$
\left\langle q_{\alpha \beta}\right\rangle_{F\left(\bar{g}^{*}\right)} \stackrel{!}{=} \bar{g}_{\alpha \beta}^{*}
$$

Here $F$ is defined via a stationarity condition referring to the full quantum dynamics and hence implicitly to the underlying state, see section 1.2. Equation (1.1) can thus be viewed as selecting a class of state dependent backgrounds $\bar{g}_{\alpha \beta}^{*}$ such that the average of the quantum metric in the state co-determined by $\bar{g}_{\alpha \beta}^{*}$ coincides with $\bar{g}_{\alpha \beta}^{*}$. For definiteness we formulated (1.1) in terms of the metric, assuming in particular that $\left\langle q_{\alpha \beta}\right\rangle_{F(\bar{g})}$ is well-defined. This assumption is dispensible, however, as one could rephrase the above construction with whatever (nonlocal) composites or observables $\mathcal{O}$ one decides to work with: given a family of $\overline{\mathcal{O}}$ 's containing the information about the background metric $\bar{g}$ deemed relevant, one can for any initially prescribed set of their values define a coarse graining operation relative to it and use the coarse graining to construct the functional averages \langle\rangle$_{F(\overline{\mathcal{O}})}$, depending parametrically on the $\overline{\mathcal{O}}$ values. In a second step one can stipulate the counterpart of $(1.1)$, i.e. $\langle\mathcal{O}\rangle_{F(\overline{\mathcal{O}})}=\overline{\mathcal{O}}$, which dynamically adjusts the values $\overline{\mathcal{O}}$ to the selfconsistent ones $\overline{\mathcal{O}}^{*}$. In formulating $(1.1)$ we assumed that the 'infrared problem' has been solved, in particular that the full averages used for the adjustment contain information also about the infrared degrees of freedom and are well-defined. The same adjustment could, however, be done using scale dependent Wilsonian averages at some scale infrared cutoff scale $\mu$, see Section 1.2.

With respect to a given coarse graining operation one can ask whether the flow of actions or couplings has a fixed point. The existence of a fixed point is the raison d'être for the universality properties (in the statistical field theory sense) which eventually are 'handed down' to the physics in the low energy regime. By analogy with other field theoretical systems one should probably not expect that the existence (or nonexistence) of a (nonGaussian) fixed point will be proven with mathematical rigor in the near future. From a physics viewpoint, however, it is the high degree of universality ensued by a fixed point that matters, rather than the existence in the mathematical sense. For example nonabelian gauge theories appear to have a (Gaussian) fixed point 'for all practical 
purposes', while their rigorous construction as the continuum limit of a lattice theory is still deemed a 'millennium problem'. In the case of quantum gravity we shall survey in Section 1.3 various pieces of evidence for the existence of a (non-Gaussian) fixed point.

Accepting the existence of a (non-Gaussian) fixed point as a working hypothesis one is led to determine the structure of its unstable manifold. Given a coarse graining operation and a fixed point of it, the stable (unstable) manifold is the set of all points connected to the fixed point by a coarse graining trajectory terminating at it (emanating from it). It is not guaranteed though that the space of actions can in the vicinity of the fixed point be divided into a stable and an unstable manifold; there may be trajectories which develop singularities or enter a region of coupling space deemed unphysical for other reasons and thus remain unconnected to the fixed point. The stable manifold is the innocuous part of the problem, it is the unstable manifold which is crucial for the construction of a continuum limit. By definition it is swept out by flow lines emanating from the fixed point, the so-called renormalized trajectories. Points on such a flow line correspond to actions or measures which are called perfect in that they can be used to compute continuum answers for physical quantities even in the presence of an ultraviolet (UV) cutoff, like one which discretizes the base manifold [73]. In practice the unstable manifold is not known and renormalized trajectories have to be identified approximately by a tuning process. What is easy to determine is whether in a given expansion "sum over coupling times interaction monomial" a coupling will be driven away from the value the corresponding coordinate has at the fixed point after a sufficient number of coarse graining steps (in which case it is called relevant) or will move towards this fixed point value (in which case it is called irrelevant). Note that this question can be asked even for trajectories which are not connected to the fixed point. The dimension of the unstable manifold equals the number of independent relevant interaction monomials that are 'connected' to the fixed point by a (renormalized) trajectory.

In quantum gravity traditionally the Einstein-Hilbert action is taken as the microscopic action. Perturbatively this action is not connected to a fixed point, not even to the perturbative Gaussian one. The question whether or not the situation improves in a nonperturbative formulation has been mostly addressed in discretized formulations, see $[70,5]$ and references therein. The discretized action used then may no longer have a naive (classical) continuum limit reproducing the Einstein-Hilbert action, but it is still labelled by two bare parameters. Conceptually one can assign to the discretized twoparametric measure a microscopic action in the above sense by requiring that combined with the regularized continuum measure [22] it reproduces approximately the same correlation functions. The microscopic action defined that way would presumably be different from the Einstein-Hilbert action but it would still contain only two tunable parameters. Presupposing again the existence of a fixed point, this type of construction relies on the hope that the non-naive discretization procedure adopted gets all but two coordinates of the unstable manifold automatically right. We refer to $[70,5]$ for the numerical evidence.

In the present context a counterpart of these constructions starting from a perturbatively (weakly or strictly) microscopic renormalizable action (see Section 2) would seem 
more promising. The tuning to the unstable manifold then is more complicated, but perturbation theory (or other expansion techniques) can be used as a guideline, both analytically and for the extrapolation of numerical results.

Typically the unstable manifold is indeed locally a manifold, though it may have cusps. Although ultimately it is only the unstable manifold that matters for the construction of a continuum limit, relevant couplings which blow up somewhere inbetween may make it very difficult to successfully identify the unstable manifold. In practice, if the basis of interaction monomials in which this happens is deemed natural and a change of basis in which the pathological directions could simply be omitted from the space of actions is very complicated, the problems caused by such a blow up may be severe. An important issue in practice is therefore whether in a natural basis of interaction monomials the couplings are 'safe' from such pathologies and the space of actions decomposes in the vicinity of the fixed point neatly into a stable and an unstable manifold. This regularity property is one aspect of "asymptotic safety", as we shall see below.

A second limitation appears in infinite dimensional situations. Whenever the coarse graining operates on an infinite set of potentially relevant interaction monomials convergence issues in the infinite sums formed from them may render formally equivalent bases inequivalent. In this case the geometric picture of a (coordinate independent) manifold breaks down or has to be replaced by a more refined functional analytic framework. An example of a field theory with an infinite set of relevant interaction monomials is QCD in a lightfront formulation [123] where manifest Lorentz and gauge invariance is given up in exchange of other advantages. In this case it is thought that there are hidden dependencies among the associated couplings so that the number of independent relevant couplings is finite and the theory is eventually equivalent to conventional QCD. Such a reduction of couplings is nontrivial because a relation among couplings has to be preserved under the renormalization flow. In quantum gravity related issues arise to which we turn later.

As an interlude let us mention the special role of Newton's constant in a diffeomorphism invariant theory with a dynamical metric. Let $S[q$, matter $]$ be any local action, where $q=\left(q_{\alpha \beta}\right)_{1 \leq \alpha, \beta \leq d}$ is the ('quantum') metric entering the (regularized) functional integral and the "matter" fields are not scaled when the metric is. Constant rescalings of the metric then give rise to a variation of the Lagrangian which vanishes on shell:

$$
\left.\frac{d}{d \omega^{2}} S\left[\omega^{2} q, \text { matter }\right]\right|_{\omega=1}=\int d x \sqrt{q} q_{\alpha \beta} \frac{\delta S[q, \text { matter }]}{\delta q_{\alpha \beta}} .
$$

As a consequence one of the coupling parameters which in the absence of gravity would be essential (i.e. a genuine coupling) becomes inessential (i.e. can be changed at will by a redefinition of the fields). The running of this parameter, like that of a wave function renormalization constant, has no direct significance. If the pure gravity part contains the usual Ricci scalar term, $\sqrt{q} R(q)$, the parameter that becomes inessential may be taken as its prefactor $Z_{N}$. Up to a dimension dependent coefficient it can be identified 
with the inverse of Newton's constant $Z_{N}^{-1} \sim G_{\text {Newton. }}$ It is also easy to see that in a background field formalism $\omega$ sets the overall normalization of the spectral/momentum values. Hence in a theory with a dynamical metric the three (conceptually distinct) inessential parameters: overall scale of the metric, the inverse of Newton's constant, and the overall normalization of the spectral/momentum values are in one-to-one correspondence; see section 2.1 for details. For definiteness let us consider the running of Newton's constant here.

Being inessential, the quantum field theoretical running of $G_{\text {Newton }}$ has significance only relative to the running coefficient of some reference operator. The most commonly used choice is a cosmological constant term $\tilde{\Lambda} \int d x \sqrt{q}$. Indeed

$$
G_{\text {Newton }} \tilde{\Lambda}^{\frac{d-2}{d}}=: \text { const } \tau(\mu)^{2 / d}
$$

is dimensionless and invariant under constant rescalings of the metric [83]. The associated essential coupling $\tau(\mu)$ is in the present context assumed to be asymptotically safe, i.e. $\sum_{\mu_{0} \leq \mu \leq \infty} \tau(\mu)<\infty, \lim _{\mu \rightarrow \infty} \tau(\mu)=\tau_{*}$, where here $0<\tau_{*}<\infty$. Factorizing

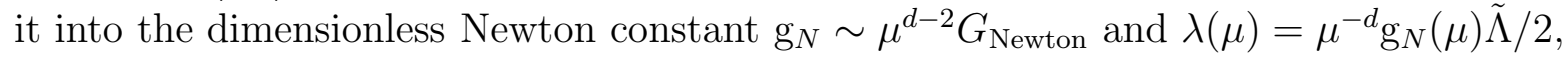
there are two possibilities: One is that the scheme choices are such that both $\mathrm{g}_{N}$ and $\lambda$ behave like asymptotically safe couplings, i.e. satisfy (1.5) below. This is advantageous for most purposes. The second possibility is realized when a singular solution for the flow equation for $\mathrm{g}_{N}$ is inserted into the flow equation for $\lambda$. This naturally occurs when $G_{\text {Newton }}$, viewed as an inessential parameter, is frozen at a prescribed value, say $\left[G_{\text {Newton }}\right]^{1 /(d-2)}=M_{\mathrm{Pl}} \approx 1.4 \times 10^{19} \mathrm{GeV}$, which amounts to working with Planck units. Then the $\mathrm{g}_{N}$ flow is trivial, $\mathrm{g}_{N}(\mu) \sim\left(\mu / M_{\mathrm{Pl}}\right)^{d-2}$, but the flow equation for $\lambda$ carries an explicit $\mu$-dependence [119]. By and large both formulations are mathematically equivalent, see section 2.1. For definiteness we considered here the cosmological constant term as a reference operator, but many other choices are possible. In summary, the dimensionless Newton constant can be treated either as an inessential parameter (and then frozen to a constant value) or as a quasi-essential coupling (in which case it runs and assumes a finite positive asymptotic value).

The unstable manifold of a fixed point is crucial for the construction of a continuum limit. The fixed point itself describes a strictly scale invariant situation. More precisely the situation at the fixed point is by definition invariant under the chosen coarse graining (i.e. scale changing) operation. In particular any dependence on an ultraviolet cutoff must drop out 'at' the fixed point, which is why fixed points are believed to be indispensable for the construction of a scaling limit. If one now uses a different coarse graining operation the location of the fixed point will change in the given coordinate system provided by the essential couplings. One aspect of universality is that all field theories based on the fixed points referring to different coarse graining operations have the same long distance behavior.

This suggests to introduce the notion of a continuum limit as an 'equivalence class' of scaling limits in which the physical quantities become strictly independent of the 
UV cutoff, largely independent of the choice of the coarse graining operation, strictly independent of the choice of gauge slice and, ideally, invariant under local reparameterizations of the fields.

In the framework of statistical field theories one distinguishes between two construction principles, a massless scaling limit and a massive scaling limit. In the first case all the actions/measures on a trajectory emanating from the fixed point describe a scale invariant system, in the second case this is true only for the action/measure at the fixed point. In either case the unstable manifold of the given fixed point has to be at least one dimensional. Here we shall exclusively be interested in the second construction principle. Given a coarse graining operation and a fixed point of it with a nontrivial unstable manifold a scaling limit is then constructed by 'backtracing' a renormalized trajectory emanating from the fixed point. The number of parameters needed to specify a point on the unstable manifold gives the number of possible scaling limits - not all of which must be physically distinct, however.

In this context it should be emphasized that the number of relevant directions in a chosen basis is not directly related to the predictive power of the theory. A number of authors have argued in the effective field theory framework that even theories with an infinite number of relevant parameters can be predictive $[92,13,27]$. This applies all the more if the theory under consideration is based on a fixed point, and thus not merely effective. One reason lies in the fact the number of independent relevant directions connected to the fixed point might not be known. Hidden dependencies would then allow for a (genuine or effective) reduction of couplings $[159,113,123,9,13]$. For quantum gravity the situation is further complicated by the fact that generic physical quantities are likely to be related only nonlocally and nonlinearly to the metric. What matters for the predictive power is not the total number of relevant parameters but how the observables depend on them. To illustrate the point imagine a (hypothetical) case where $n^{2}$ observables are injective functions of $n$ relevant couplings each:

$$
\mathcal{O}_{i}\left(\mathrm{~g}_{1}, \ldots, \mathrm{g}_{n}\right), \quad i=1, \ldots, n^{2}
$$

Then $n$ measurements will determine the couplings, leaving $n^{2}-n$ predictions. This gives plenty of predictions, for any $n$, and it remains true in the limit $n \rightarrow \infty$, despite the fact that one then has infinitely many relevant couplings. This example may be seen as a mathematical abstraction of the reason why effective field theories (or renormalizable ones with a UV cutoff kept) are predictive. The $\mathcal{O}_{i}$ 's may depend on additional couplings, but if this dependence is quantitatively sufficiently suppressed the situation is qualitatively as in the example.

Initially infinitely many essential couplings arise when a perturbative treatment of Quantum Gravidynamics is based on a $1 / p^{2}$ type propagator. Perturbation theory can be seen as a degenerate special case of the general framework described before. Depending on the structure of the coupling flow the associated perturbative Gaussian fixed point does or does not reflect a Gaussian fixed point proper. In the case of gravity, as first 
advocated by Gomis and Weinberg [66], the use of a $1 / p^{2}$ type graviton propagator in combination with higher derivative terms avoids the problems with unitarity that occur in other treatments of higher derivative theories. Consistency requires that quadratic counterterms (those which contribute to the propagator) can be absorbed by field redefinitions. This can be seen to be the case [8] either in the absence of a cosmological constant term or when the background spacetime admits a metric with constant curvature. The price to pay for the $1 / p^{2}$ type propagator is that all nonquadratic counterterms have to be included in the bare action, so that independence of the UV cutoff can only be achieved with infinitely many essential couplings, but it can be [66]. In order to distinguish this from the familiar notion of perturbative renormalizability with finitely many couplings we shall call such theories (perturbatively) weakly renormalizable. The above results then show the existence of a "weakly renormalizable" but "propagator unitary" Quantum Gravidynamics based on a perturbative Gaussian fixed point.

The beta functions for this infinite set of couplings are presently unknown. If they were known, expectations are that at least a subset of the couplings would blow up at some finite momentum scale $\mu=\mu_{\text {term }}$ and would be unphysical for $\mu>\mu_{\text {term }}$. In this case the computed results for physical quantities ("reaction rates") are likely to blow up likewise at some (high) energy scale $\mu=\mu_{\text {term }}$.

This illustrates Weinberg's concept of asymptotic safety. To quote from [152]: "A theory is said to be asymptotically safe if the essential coupling parameters approach a fixed point as the momentum scale of their renormalization point goes to infinity". Here 'the' essential couplings $g_{i}$ are those which are useful for the absorption of cutoff dependencies, i.e. not irrelevant ones. The momentum scale is the above $\mu$, so that the condition amounts to having nonterminating trajectories for the $\mathrm{g}_{i}$ 's with a finite limit:

$$
\sup _{\mu_{0} \leq \mu \leq \infty} \mathrm{g}_{i}(\mu)<\infty, \quad \lim _{\mu \rightarrow \infty} \mathrm{g}_{i}(\mu)=\mathrm{g}_{i}^{*}<\infty
$$

for some $i$-independent $\mu_{0}$. In other words in an asymptotically safe theory the above blow up in the couplings and hence in physical observables does not occur. We suggest to call couplings satisfying (1.5) asymptotically safe. As a specification one should add [152]: "Of course the question whether or not an infinity in coupling constants betokens a singularity in reaction rates depends on how the coupling constants are parameterized. We could always adopt a perverse definition (e.g. $\left.\tilde{g}(\mu)=\left(g_{*}-g(\mu)\right)^{-1}\right)$ such that reaction rates are finite even at an infinity of the coupling parameters. This problem can be avoided if we define the coupling constants as coefficients in a power series expansion of the reaction rates themselves around some physical renormalization point".

A similar remark applies to the signs of coupling constants. When defined through physical quantities certain couplings or coupling combinations will be constrained to be positive. For example in a (nongravitational) effective field theory this constrains the couplings of a set of leading power counting irrelevant operators to be positive [2, 134]. In an asymptotically safe theory similar constraints are expected to arise and are crucial 
for its physics viability.

Note that whenever the criterion for asymptotic safety is met all the relevant couplings lie in the unstable manifold of the fixed point (which is called the "UV critical surface" in [152], p.802, a term now usually reserved for the surface of infinite correlation length). The regularity property described earlier is then satisfied and the space of actions decomposes in the vicinity of the fixed point into a stable and an unstable manifold.

Comparing the two perturbative treatments of Quantum Gravidynamics described earlier one sees that they have complementary advantages and disadvantages: higher derivative theories based on a $1 / p^{4}$ propagator are strictly renormalizable with couplings that are presumed to be asymptotically safe; however unphysical propagating modes are present. Defining higher derivative gravity perturbatively with respect to a $1 / p^{2}$ propagator has the advantage that all propagating modes are physical, but initially infinitely many essential couplings are needed, a subset of which is presumed to be not asymptotically safe. From a technical viewpoint the challenge of Quantum Gravidynamics lies therefore not so much in achieving renormalizability but to reconcile asymptotically safe couplings with the absence of unphysical propagating modes.

Even in the above perturbative formulations one can see heuristically how this might be feasible: both descriptions should be related through a reduction of couplings, i.e. the infinite set of couplings in the $1 / p^{2}$ formulation should be thought of as having hidden dependencies such that a nonredundant set corresponds to the finitely many safe couplings in the $1 / p^{4}$ formulation. The proper computational implementation presumably requires new (perturbative or nonperturbative) techniques.

Assuming that this can be achieved certain qualitative features such a gravitational functional integral must have can be inferred without actually evaluating it. One is the presence of anti-screening configurations, the other is a dimensional reduction phenomenon in the ultraviolet.

In nonabelian gauge theories the anti-screening phenomenon can be viewed as the physics mechanism underlying their benign high energy behavior (as opposed to abelian gauge theories, say), see e.g. [124] for an intuitive discussion. It is important not to identify "anti-screening" with its most widely known manifestation, the sign of the dominant contribution to the one-loop beta function. In an exact continuum formulation of a pure Yang-Mills theory, say, the correlation functions do not even depend on the gauge coupling. Nevertheless they indirectly do know about "asymptotic freedom" through their characteristic high energy behavior. The phenomenon is also state-dependent: it is the Yang-Mills vacuum that acts like a specific polarizable medium. In the functional integral measure this in principle comes about through the dominance of certain configurations/histories which one might also call "anti-screening".

By analogy one would expect that in a gravitational functional integral that allows for a continuum limit a similar mechanism is responsible for its benign ultraviolet behavior (as opposed to the one expected by power counting considerations with respect to a 
$1 / p^{2}$ propagator, say). As in the Yang-Mills case a certain class of states will act like a polarizable, predominantly "antiscreening" medium. Importantly, since a preferred ground state is unlikely to exist in quantum gravity, one can take advantage of the ensued ambiguity to select the class of states appropriately. In a functional integral the state dependence can be encoded in boundary terms for the microscopic action, so that a corresponding ambiguity in the definition of the functional integral will result. Some insight into the nature of the gravitational antiscreening mechanism can be gained from a hamiltonian formulation of the functional integral but a proper understanding of the interplay between the class of states, the dominant geometries/histories, and the renormalization properties in the ultraviolet remains to be found. Nevertheless it is clearly legitimate to utilize the beforementioned ambiguities so as to faciltate the construction of a continuum limit. For simplicity we shall refer to such an adjustment as the implementation of an "anti-screening constraint".

In a discretized functional integral the dominance of antiscreening configurations/histories would by definition be responsable for the benign ultraviolet properties associated with a a non-Gaussian fixed point. Conversely understanding the nature of these antiscreening geometries/histories might help to design good discretizations. A discretization of the gravitational functional integral which allows for a continuum limit might also turn out to exclude or dynamically disfavor configurations that are taken into account in other, off-hand equally plausible, discretizations. Compared to such a naive discretization it will look as if a constraint on the allowed configurations/histories has been imposed. A useful analogy is the inclusion of a causality constraint in the definition of the (formal Euclidean) functional integral originally proposed by Teitelboim [142, 143], and recently put to good use in the framework of dynamical triangulations [4]. Just as the inclusion of a good causality constraint is justified retroactively, so would be the inclusion of a suitable "antiscreening" constraint.

A second qualitative property of a gravitational functional integral where the continuum limit is based on a non-Gaussian fixed point is a dimensional reduction of the residual interactions in the UV. There are several arguments for this phenomenon which we describe in section 3. Perhaps the simplest one is based on the large anomalous dimensions at a non-Gaussian fixed point and runs as follows: (We present here a model-independent variant [106] of the argument used in [94]). Suppose that the unkown microscopic action is local and reparameterization invariant. The only term containing second derivatives then is the familiar Einstein-Hilbert term $\int d x \sqrt{q} R(q)$ of mass dimension $2-d$ in $d$ dimensions, if the metric is taken dimensionless. As explained before the dimensionful running prefactor $Z_{N}$ ( $N$ for "Newton") multiplying it plays a double role, once as a wave function renormalization constant and once as a quasi-essential coupling $\mathrm{g}_{N}(\mu)$. Both aspects are related as outlined before; in particular

$$
Z_{N}(\mu)=\frac{\mu^{d-2}}{\mathrm{~g}_{N}(\mu)} .
$$


Here $\mathrm{g}_{N}$ is a dimensionless coupling which is treated as "quasi-essential" and whose running may also depend on all the other couplings (gravitational and non-gravitational) made dimensionless by taking out a suitable power of $\mu$. The short distance behavior of the propagator will now be governed by the "anomalous dimension" $\eta_{N}=-\mu \partial_{\mu} \ln Z_{N}(\mu)$, by the usual field theoretical arguments, say, via the Callan-Symanzik equation for the effective action. On the other hand the flow equation for $\mathrm{g}_{N}$ can be expressed in terms of $\eta_{N}$ as

$$
\mu \partial_{\mu} \mathrm{g}_{N}=\left[d-2+\eta_{N}\left(\mathrm{~g}_{N}, \text { other }\right)\right] \mathrm{g}_{N},
$$

where we schematically indicated the dependence on the other dimensionless couplings. If this flow equation now has a nontrivial fixed point $\infty>\mathrm{g}_{N}^{*}>0$, the only way how the right hand side can vanish is for $\eta_{N}\left(\mathrm{~g}_{N}^{*}\right.$, other $)=2-d$, irrespective of the detailed behavior of the other couplings as long as no blow-up occurs. This is a huge anomalous dimension. For a graviton "test propagator" (see below) the key property of $\eta_{N}$ is that it gives rise to a high momentum behavior of the form $\left(p^{2}\right)^{-1+\eta_{N} / 2}$ modulo logarithms, or a short distance behavior of the form $\left(\sqrt{x^{2}}\right)^{2-d-\eta_{N}}$ modulo logarithms. Keeping only the leading part the vanishing power at $\eta_{N}=2-d$ translates into a logarithmic behavior, $\ln x^{2}$, formally the same as for massless (scalar) propagators in a two-dimensional field theory. We shall comment on potential pitfalls of such an argument below.

In accordance with this argument a $1 / p^{4}$ type propagator goes hand in hand with a nonGaussian fixed point for $\mathrm{g}_{N}$ in two other computational settings: in strictly renormalizable higher derivative theores (see section 2.2) and in the $1 / N$ expansion [145, 146, 135]. In the latter case a nontrivial fixed point goes hand in hand with a graviton propagator whose high momentum behavior is of the form $1 /\left(p^{4} \ln p^{2}\right)$, in four dimensions, and formally $1 / p^{d}$ in $d$ dimensions.

The fact that a large anomalous dimension occurs at a non-Gaussian fixed point was first observed in in the context of the $2+\epsilon$ expansion [83, 84] and then noticed in computations based on truncated flow equations [94]. The above variant of the argument shows that no specific computational information enters. It highlights what is special about the Einstein-Hilbert term (within the class of local gravitational actions): it is the kinetic (second derivative) term itself which carries a dimensionful coupling. Of course one could assign to the metric a mass dimension 2, in which case Newton's constant would be dimensionless. However one readily checks that then the wave function renormalization constant of a standard matter kinetic term acquires a mass dimension $d-2$ for bosons and $d-1$ for fermions, respectively. Assuming that the dimensionless parameter associated with them remains nonzero as $\mu \rightarrow \infty$ one can repeat the above argument and finds now that all matter propagators have a $1 / p^{d}$ type high momentum behavior, or a logarithmic short distance behavior. It is this universality which justifies to attribute the modification in the short distance behavior of the fields to a modification of the underlying (random) geometry. This may be viewed as a specific variant of the old expectation that gravity acts as a short distance regulator.

Let us stress that while the anomalous dimension always governs the UV behavior in 
the vicinity of a (UV) fixed point, it is in general not related to the geometry of field propagation, see [91] for a discussion in QCD. What is special about gravity is ultimately that the propagating field itself determines distances. In the context of the above argument this is used in the reshuffling of the soft UV behavior to matter propagators. The propagators used here should be viewed as "test propagators", not as physical ones. One transplants the information in $\eta_{N}$ derived from the gravitational functional integral into a conventional propagator on a (flat or curved) background spacetime. The reduced dimension two should be viewed as an "interaction dimension" specifying roughly the (normalized) number of independent degrees of freedom a randomly picked one interacts with.

The same conclusion $\left(1 / p^{d}\right.$ propagators or interaction dimension 2$)$ can be reached in a number of other ways as well, which are described in section 3. A more detailed understanding of the microstructure of the random geometries occuring in an asymptotically safe functional integral remains to be found.

Accepting this dimensional reduction as a working hypothesis it is natural to ask whether there exists a two-dimensional field theory which provides a quantitatively accurate ('effective') description of this extreme UV regime. Indeed, one can identify a number of characteristics such a field theory should have, using only the main ideas of the scenario, see the end of Section 3. The asymptotic safety of such a field theory would then strongly support the corresponding property of the full theory and the selfconsistency of the scenario. In summary, we have argued that the qualitative properties of the gravitational functional integral in the extreme ultraviolet follow directly from the previously highlighted principles: the existence of a nontrivial UV fixed point, asymptotic safety of the couplings, and antiscreening. Moreover these UV properties can be probed for selfconsistency.

\subsection{Coarse graining and dynamically adjusted background data}

Since renormalization implicitly (in perturbation theory) or explicitly (in the KadanoffWilson framework) depends on the choice of a coarse graining operation one is in a quantum gravity context lead to address the question "with respect to what" field configurations are coarsely or finely grained. The piecemeal evaluation of the functional integral (decomposition of the presumed 'critical' problem into 'subcritical' ones) requires a physically motivated notion of the slicing. For statistical field theories on a non-dynamical background the spectrum of a covariant differential operator (generalized momenta) can be used. In quantum gravity the determination of an averaged geometry is part of the dynamical problem, and one has to proceed differently. The retroactive dynamical adjustment of initially prescribed background data provides a natural generalization. The principle has already been outlined in the discussion around Eq. (1.1). 
Here we describe the construction in somewhat more detail using the background effective action formalism, see $[78,1,30]$ for the latter. In this formalism the effective action $\Gamma$ of a scalar field theory becomes a (highly nonlocal) functional of two fields, $\Gamma=\Gamma[f, \bar{\chi}]$. The second field is the initially prescribed background field $\bar{\chi}$, the first can be interpreted as the source dependent average $\langle\chi-\bar{\chi}\rangle_{J_{*}}$ of the quantum field $\chi$ shifted by $-\bar{\chi}$, where the source $J_{*}$ is given by $J_{*}[f ; \bar{\chi}]=\delta \Gamma[f ; \bar{\chi}] / \delta f$. Switching off the source, $J_{*}[f ; \bar{\chi}]=0$, correlates both fields and one may assume that locally (in function space) one can be expressed as a functional of the other. We write the relation as $\bar{\chi}=F(f+\bar{\chi})$, so that $F=$ id corresponds to $f=0$, and assume that it can be solved locally for $f$, i.e. $f=-\bar{\chi}+F^{-1}(\bar{\chi})$. Then $\bar{\Gamma}[\phi]:=\Gamma[\phi-\bar{\chi} ; F(\phi)]$, obeys $([106,110]) \delta \bar{\Gamma}[\phi] / \delta \phi=\langle\delta S / \delta \bar{\chi}\rangle_{J_{*}=0} \delta F / \delta \phi$, where the derivative of the action $S$ is taken with respect to the explicit background dependence, if any. From the viewpoint of the underlying functional integral a dynamically adjusted background $\bar{\chi}=F(f+\bar{\chi})$ is optimal with regard to a small field expansion around it, where "small", however, now means "selfconsistently small with respect to the full quantum dynamics".

This construction can be transferred to gravity, where the dynamically adjusted background metric can in addition be used to define an intrinsic coarse graining scale. As remarked before the use of a background metric as opposed to other, more specific, background data is dispensible, for concreteness we use here the metric itself, both as a dynamical variable, $q_{\alpha \beta}$, in the functional integral and to specify the background data needed. This leads to an effective action $\Gamma[f ; \bar{g}, \ldots]$ which is a reparameterization invariant functional of two symmetric second rank tensors. The second, $\bar{g}_{\alpha \beta}$, is an initially independently prescribed "background metric". The first, $f_{\alpha \beta}$, is interpreted as an initially source dependent average $\left\langle q_{\alpha \beta}-\bar{g}_{\alpha \beta}\right\rangle_{J_{*}}$ of the dynamical metric $q_{\alpha \beta}$ shifted by $-\bar{g}_{\alpha \beta}$, where the source $J_{*}$ is given by $J_{*}[f ; \bar{g}]=\delta \Gamma[f ; \bar{g}] / \delta f$. The dots in $\Gamma[f ; \bar{g}, \ldots]$ indicate other fields, dual to ghost sources, which are inessential to the discussion. Switching off the source, $J_{*}[f, \bar{g}]=0$, now correlates $f$ with $\bar{g}$ and as before we may assume that a functional relation $\bar{g}=F(f+\bar{g})$ with inverse $f=-\bar{g}+F^{-1}(\bar{g})$ holds, at least locally in the space of metrics. Then $\bar{\Gamma}[g]:=\Gamma[g-\bar{g}, F(g)]$, obeys [106]

$$
\frac{\delta \bar{\Gamma}[g]}{\delta g_{\alpha \beta}}=\left\langle\frac{\delta S}{\delta \bar{g}_{\gamma \delta}}\right\rangle_{J_{*}=0} \frac{\delta F_{\gamma \delta}}{\delta g_{\alpha \beta}}
$$

where the derivative of the action $S$ is taken with respect to the explicit background dependence. Starting with a reparameterization invariant microscopic action $S_{0}[q]$, the gauge fixing and ghost terms will introduce such an explicit dependence on $\bar{g}_{\alpha \beta}$; schematically $S[q ; \bar{g}]=S_{0}[q]+S_{\text {g.f. }}[q-\bar{g} ; \bar{g}]+S_{\text {ghost }}[q-\bar{g} ; \bar{g}]$. The solutions of (1.8) contain information about: the source-free condition $J_{*}[f ; \bar{g}]=0$, about the state vector underlying the functional integral through the choice of boundary terms, and about the choice of gauge-slice. We now comment on each of these dependencies successively:

The construction of the extremizing sources $J_{*}[f ; \bar{g}]$ entering the definition of $\Gamma[f ; \bar{g}, \ldots]$ (as the Legendre transform of $W[J ; \bar{g}, \ldots]$, the generating functional of connected cor- 
relation functions) is usually done within a formal power series ansatz. This gives a solution $J_{*}[f ; \bar{g}](x)=\sum_{n \geq 1} j_{n}\left(x, x_{1}, \ldots, x_{n}\right) f\left(x_{1}\right) \ldots f\left(x_{n}\right)$, where the $j_{n}$ 's can be expressed in terms of the $f$ moments $W^{(n)}$ of $W$, 'amputated' with the exact $\Gamma^{(2)}[f ; \bar{g}]=$ $\delta \Gamma[f ; \bar{g}] /(\delta f \delta f)$. Clearly $J_{*} \equiv 0$ iff $f \equiv 0$ within a formal power series ansatz. This amounts to $F=$ id and the dynamically adjusted background coincides with the prescribed one. Conversely, in order to get a genuine dynamical adjustment, one has to go beyond a formal power series ansatz. Assuming $f=-\bar{g}+F^{-1}(\bar{g})$ one gets $\left\langle q_{\alpha \beta}\right\rangle_{J_{*}=0}=F_{\alpha \beta}^{-1}(\bar{g})$, The functional $F^{-1}$ here contains the dynamical information inherited from the full $\Gamma$ via $\delta \Gamma[f ; \bar{g}, \ldots] / \delta f=0$. The right hand side of (1.1) can now be viewed as a new background $\bar{g}_{\alpha \beta}^{*}$ and the parameteric dependence of the state \langle\rangle$_{J_{*}=0}$ can be relabelled to \langle\rangle$_{F(\bar{g})}$. The equation characterizing the class of dynamically adjusted backgrounds then becomes

$$
\left\langle q_{\alpha \beta}\right\rangle_{F\left(\bar{g}^{*}\right)}=\bar{g}_{\alpha \beta}^{*},
$$

as anticipated in (1.1).

The notion of a state is implicitly encoded in the effective action. Recall that the standard effective action for a scalar field theory, when evaluated on a given time-independent function $\varphi^{i}=\left\langle\chi^{i}\right\rangle$, is proportional to the minimum value of the Hamiltonian $H$ in that part of the Hilbert space spanned by normalizable states $|\psi\rangle$ satisfying $\left\langle\psi\left|\chi^{i}\right| \psi\right\rangle=\varphi^{i}$. A similar interpretation holds formally for the various background effective actions [34]. In a functional integral formulation the information about the state can be encoded in suitable (though often not explicitly known) boundary terms for the microscopic action. An alternative way to see that $\Gamma$ in principle also encodes the information about the underlying state vector, is via reconstruction. Let $\Gamma^{(n)}, n \geq 2$, be the vertex functions associated with $\Gamma$, i.e.

$$
\Gamma^{(n)}\left(x_{1}, \ldots, x_{n} ; g\right):=\left.\frac{\delta}{\delta g\left(x_{1}\right)} \ldots \frac{\delta}{\delta g\left(x_{n}\right)} \Gamma[g-\bar{g} ; \bar{g}, \ldots]\right|_{\bar{g}=F(g)}
$$

In a flat space quantum field theory the Wightman or Osterwalder-Schrader reconstruction procedures would allow one to (re-)construct the state space and field operators from knowledge of the $\Gamma^{(n)}$. In a quantum gravity context little is known about the feasibility of such a reconstruction from e.g. the vertex functions (1.10). The use of metric correlators (or quantities tentatively interpreted as such) may also not be ideal from the viewpoint of such a reconstruction. One would expect that correlators of (nonlocal) quantities closer to (Dirac) observables are better suited for a reconstruction. Returning to $\Gamma$, one should think of it as a functional of both the selected state and of the fields. The selected state will indirectly (co-)determine the space of functionals on which the renormalization flow acts. For example the type of nonlocalities which actually occur in $\Gamma$ should know about the fact that $\Gamma$ stems from a microscopic action suited for the appropriate notion of positivity and from a physically acceptable state.

The notion of a physically acceptable state is another unexplored issue in this context. In conventional flat space quantum field theories there is a clear-cut notion of a ground 
state and of the physical state space based on it. Already in quantum field theories on curved but non-dynamical spacetimes a preferred vacuum is typically absent and physically acceptable states have to be selected by suitable conditions (like, for example, the well-known Hadamard condition imposed on the short distance behavior of the two point function, which for free quantum field theories in curved spacetime selects states with desirable stability properties.) In quantum gravity the formulation of analogous selection criteria is an open problem. As a tentative example we mention the condition [106]

$$
\begin{aligned}
& \left\langle P_{q}(T)\right\rangle \sim T^{-d / 2}, \quad T \rightarrow \infty, \\
& P_{q}(T):=\int d x \sqrt{q} \exp \left(T \Delta_{q}\right)(x, x) .
\end{aligned}
$$

Here $\Delta_{q}$ is the Laplace-Beltrami operator of a (pseudo-) riemannian metric $q_{\alpha \beta}$, and $\exp \left(T \Delta_{q}\right)(x, y)$ is the associated heat kernel. When $q=\eta$ is flat $P_{\eta}(T)$ decays like $T^{-d / 2}$ for $T \rightarrow \infty$. The condition (1.11) therefore indirectly characterizes a class of states which favor geometries that are smooth and almost flat on large scales.

Finally we comment on the gauge dependence of $\Gamma$ or $\bar{\Gamma}$. The right hand side of (1.8) renders the dependence of $\bar{\Gamma}$ on the choice of gauge slice manifest. Had a (technically more complicated) Vilkovisky-deWitt type effective action [158, 126, 117] been used this dependence should be absent. As an approximative shortcut one can continue to work with the previous background effective action and consider solutions $\check{g}$ of $\delta \bar{\Gamma}[g] / \delta g_{\alpha \beta}=0$, which retroactively minimize the dependence on the choice of gauge. This condition will be used later on. Since $\bar{\Gamma}$ is highly nonlocal the identification of physical solutions of (1.8) or $\delta \bar{\Gamma}[g] / \delta g_{\alpha \beta}=0$, is a nontrivial problem. See e.g. [16] for examples based on an anomaly induced part of an effective action. The previous discussion suggests a partial characterization, namely those solutions of (1.8) should be regarded as physical which are associated with physically acceptable states.

The use of a dynamically adjusted background geometry has the additional advantage of allowing one to introduce an intrinsic coarse graining scale. Let $\bar{g}_{\alpha \beta}$ be again an initially prescribed background geometry. Let $\mu$ denote an (unphysical) scale parameter which schematically cuts off modes whose average extension with respect to $\bar{g}_{\alpha \beta}$ is larger than $\mu^{-1}$. Clearly there is a large degree of arbitrariness in defining such a mode cut-off and for each choice there is an effective action $\Gamma_{\mu}[f, \bar{g}]$ containing mostly the dynamical information about modes larger than $\mu$. In a two step procedure one can now replace $\mu$ with an intrinsic coarse graining scale. In a first step $\bar{g}_{\alpha \beta}$ is replaced with a dynamically adjusted background $\check{g}_{\mu}$ solving the counterpart of (1.8) for $\Gamma_{\mu}$. In a second step one considers the spectrum $\left\{\mathcal{E}_{\omega}\left(\check{g}_{\mu}\right) \mid \omega \in S \subset \mathbb{R}\right\}$ of a covariant differential operator, say $\Delta_{\check{g}_{\mu}}$, built from $\check{g}_{\mu}$. The implicit equation

$$
\mu^{2}=\mathcal{E}_{\omega}\left(\check{g}_{\mu}\right)
$$

then determines $\mu=\mu(\omega)$ or $\mu=\mu(\mathcal{E})$ and hence allows one to replace $\mu$ with the 
spectral scale $\mathcal{E}$ intrinsic to the dynamically adjusted background.

\subsection{Evidence for asymptotic safety}

Presently the evidence for asymptotic safety in quantum gravity comes from the following very different computational settings: (a) the $2+\epsilon$ expansion, (b) perturbation theory of higher derivative theories and a large $\mathrm{N}$ expansion in the number of matter fields, (c) the study of symmetry truncations, and (d) that of truncated functional flow equations. Arguably none of the pieces of evidence is individually compelling but taken together they make a strong case for asymptotic safety.

The results from the $2+\epsilon$ expansion were part of Weinberg's original motivation to propose the existence of a non-Gaussian fixed point. Since gravity in two and three dimensions is non-dynamical, however, the lessons for a genuine quantum gravitational dynamics are somewhat limited. Higher derivative derivative theories were known to be strictly renormalizable with a finite number of couplings, at the expense of having unphysical propagating modes, see $[138,56,15,24]$. With hindsight one can identify a non-Gaussian fixed point for Newton's constant already in this setting, see Section 2.2. The occurance of this non-Gaussian fixed point is closely related to the $1 / p^{4}$-type propagator that is used. The same happens when (Einstein- or a higher derivative) gravity is coupled to a large number $N$ of matter fields and a $1 / N$ expansion is performed. A nontrivial fixed point is found that goes hand in hand with a $1 / p^{4}$-type progagator (modulo $\operatorname{logs}$ ), which here arises from a resummation of matter selfenergy bubbles, however.

As emphasized before the challenge of Quantum Gravidynamics is not so much to achieve (perturbative or nonperturbative) renormalizability but to reconcile asymptotically safe couplings with the absence of unphysical propagating modes. Two recent developments provide complementary evidence that this might indeed be feasible. Both of these developments take into account the dynamics of infinitely many physical degrees of freedom of the four dimensional gravitational field. In order to be computationally feasible the 'coarse graining' has to be constrained somehow. To do this the following two strategies have been pursued (which we label here (c) and (d) according to the subsection in which they will be discussed below):

(c) The metric fluctuations are constrained by a symmetry requirement but the full (infinite dimensional) renormalization group dynamics is considered. This is the strategy via symmetry reductions.

(d) All metric fluctuations are taken into account but the renormalization group dynamics is projected onto a low dimensional submanifold. Since this is done using truncations of functional renormalization group equations we shall refer to this as the strategy via truncated functional flow equations.

Both strategies (truncation in the fluctuations but unconstrained flow and unconstrained quantum fluctuations but constrained flow) are complementary. Tentatively both results 
are related by the dimensional reduction phenomenon outlined earlier.

For the remainder of this Section we now describe the pieces of evidence from the various computational settings $(\mathrm{a})-(\mathrm{d})$ mentioned. To emphasize its auxiliary role we shall write $q=\left(q_{\alpha \beta}\right)_{1 \leq \alpha, \beta \leq d}$ for the 'quantum metric' playing the role of the integration variable in the functional integral. Averages thereof or macroscopic metrics are denoted by $g_{\alpha \beta}$ and reference metrics by $\bar{g}_{\alpha \beta}$. Our curvature conventions are set by $\left(\nabla_{\alpha} \nabla_{\beta}-\nabla_{\beta} \nabla_{\alpha}\right) v^{\gamma}=R_{\delta \alpha \beta}^{\gamma} v^{\delta}$ and $R_{\alpha \beta}=R_{\alpha \gamma \beta}^{\gamma}$. For a $(-,+, \ldots,+)$ signature metric the Einstein-Hilbert and scalar field action read, $S_{\mathrm{EH}}=+(16 \pi G)^{-1} \int d x \sqrt{g}[R(g)-2 \Lambda]$ and $S=-\frac{1}{2} \int d x \sqrt{g} g^{\alpha \beta} \partial_{\alpha} \phi \partial_{\beta} \phi$, respectively. Occasionally we shall switch to Euclidean signature metrics, $(+,+, \ldots,+)$, in which case $i S_{\mathrm{EH}} \mapsto-S_{\mathrm{EH}}^{E}, i S \mapsto-S^{E}$, and the Euclidean signature Lagrangians are obtained by formally flipping the sign of the Lorentzian signature ones.

(a) Evidence from $2+\epsilon$ expansions: In the non-gravitational examples of perturbatively nonrenormalizable field theories with a non-Gaussian fixed point the non-Gaussian fixed point can be viewed as a 'remnant' of an asymptotically free fixed point in a lower dimensional version of the theory. It is thus natural to ask how gravity behaves in this respect. In $d=2$ spacetime dimensions Newton's constant $\mathrm{g}_{N}$ is dimensionless and formally the theory with the bare action $\mathrm{g}_{N}^{-1} \int d^{2} x \sqrt{q} R(q)$ is power counting renormalizable in perturbation theory. However, as the Einstein-Hilbert term is purely topological in two dimensions the inclusion of local dynamical degrees of freedom requires, at the very least, starting from $2+\epsilon$ dimensions and then studying the behavior near $\epsilon \rightarrow 0^{+}$. The resulting " $\epsilon$-expansion" amounts to a double expansion in the number of 'graviton' loops and in the dimensionality parameter $\epsilon$. Typically dimensional regularization is used, in which case the UV divergencies give rise to the usual poles in $1 / \epsilon$. Specific for gravity are however two types of complications. The first one is due to the fact that $\int d^{2+\epsilon} x \sqrt{q} R(q)$ is topological at $\epsilon=0$, which gives rise to additional "kinematical" poles of order $1 / \epsilon$ in the graviton propagator. The goal of the renormalization process is to remove both the ultraviolet and the kinematical poles in physical quantities. The second problem is that in pure gravity Newton's constant is an inessential parameter, i.e. it can be changed at will by a field redefinition. Newton's constant $\mathrm{g}_{N}$ can be promoted to a coupling proper by comparing its flow with that of the coefficient of some reference operator, which is fixed to be constant.

For the reference operator various choices have been adopted (we follow the discussion in Kawai et al [85, 83, 84, 3] with the conventions of [84]): (i) a cosmological constant term $\int d^{2+\epsilon} x \sqrt{q}$, (ii) monomials from matter fields which are quantum mechanically non-scale invariant in $d=2$, (iii) monomials from matter fields which are quantum mechanically scale invariant in $d=2$, and (iv) the conformal mode of the metric itself in a background field expansion. All choices lead to flow equation of the form

$$
\mu \frac{d}{d \mu} \mathrm{g}_{N}=\epsilon \mathrm{g}_{N}-\gamma \mathrm{g}_{N}^{2}
$$


but the coefficient $\gamma$ depends on the choice of the reference operator [85]. For all $\gamma>0$ there is a nontrivial fixed point $\mathrm{g}_{N}^{*}=\epsilon / \gamma>0$ with a one-dimensional unstable manifold. In other words $g_{N}$ is an asymptotically safe coupling in $2+\epsilon$ dimensions and the above rule of thumb suggests that this a remnant of a nontrivial fixed point in $d=4$ with respect to which $\mathrm{g}_{N}$ is asymptotically safe (see Section 1.3 for the renormalization group terminology).

Technically the non-universality of $\gamma$ arises from the before-mentioned kinematical poles. In the early papers $[60,37,152]$ the choice (i) was adopted giving $\gamma=19 / 24 \pi$, or $\gamma=(19-c) / 24 \pi$ if free matter of central charge $c$ is minimally coupled. A typical choice for (ii) is a mass term of a Dirac fermion, a typical choice for (iii) is the coupling of a four-fermion (Thirring) interaction. Then $\gamma$ comes out as $\gamma=\left(19+6 \Delta_{0}-c\right) / 24 \pi$, where $\Delta_{0}=1 / 2,1$, respectively. Here $\Delta_{0}$ is the scaling dimension of the reference operator, and again free matter of central charge $c$ has been minimally coupled. It has been argued in [85] that the loop expansion in this context should be viewed as double expansion in powers of $\epsilon$ and $1 / c$, and that reference operators with $\Delta_{0}=1$ are optimal. The choice (iv) has been pursued systematically in a series of papers by Kawai et al [83, 84, 3]. It is based on a parameterization of the metric in terms of a background metric $\bar{g}_{\mu \nu}$, the conformal factor $e^{\sigma}$, and a part $f_{\mu \nu}$ which is traceless, $\bar{g}^{\mu \nu} f_{\mu \nu}=0$. Specifically $q_{\mu \nu}=\bar{g}_{\mu \rho}\left(e^{f}\right)^{\rho}{ }_{\nu} e^{\sigma}$ is inserted into the Einstein-Hilbert action; propagators are defined (after gauge fixing) by the terms quadratic in $\sigma$ and $f_{\mu \nu}$, vertices correspond to the higher order terms. This procedure turns out to have a number of advantages. First the conformal mode $\sigma$ is renormalized differently from the $f_{\mu \nu}$ modes and can be viewed as defining a reference operator in itself; in particular the coefficient $\gamma$ comes out as $\gamma=(25-c) / 24 \pi$. Second, and related to the first point, the system has a well-defined $\epsilon$-expansion (absence of poles) to all loop orders. Finally this setting allows one to make contact to the exact (KPZ [88]) solution of two-dimensional quantum gravity in the limit $\epsilon \rightarrow 0$.

(b) Evidence from perturbation theory and large N: Modifications of the EinsteinHilbert action where fourth derivative terms are included are known to be perturbatively renormalizable [138]. A convenient parameterization is

$$
S=-\int d x \sqrt{q}\left[\tilde{\Lambda}-\frac{1}{c_{d} G_{N}} R+\frac{1}{2 s} C^{2}-\frac{\omega}{3 s} R^{2}+\frac{\theta}{s} E\right]
$$

Here $d=4+\epsilon, c_{d}$ is a constant such that $c_{4}=16 \pi, C^{2}$ is the square of the Weyl tensor and $E$ is the integrand of the Gauss-Bonnet term. The sign of the crucial $C^{2}$ coupling $s>0$ is fixed by the requirement that the Euclidean functional integral is damping. The one-loop beta functions for the (nonnegative) couplings, $s, \omega, \theta$, are known and on the basis of them these couplings are expected to be asymptotically safe. In particular $s$ is asymptotically free, $\lim _{\mu \rightarrow 0} s(\mu)=0$. The remaining couplings $\tilde{\Lambda}$ and $c_{d} G_{N}$ are made dimensionless via $g_{N}(\mu)=\mu^{d-2} c_{d} G_{N}, \lambda(\mu)=\mu^{-d} \tilde{\Lambda} g_{N}(\mu) / 2$, where $\mu$ is the renormalization scale. At $s=0$ these flow equations are compatible with 
the existence of a non-trivial fixed point for Newton's constant, $\mathrm{g}_{N}^{*} \neq 0$, see Section 2.2. The value of $g_{N}^{*}$ is highly nonuniversal but it cannot naturally be made to vanish, i.e. the nontrivial and the trivial fixed point, $\mathrm{g}_{N}^{*}=0$, do not merge. The rationale for identifying a nontrivial fixed point by perturbative means is explained in Appendix A1. The benign renormalizability properties seen in this framework are due to the $1 / p^{4}$ type propagator in combination with diffeomorphism invariance, at the expense of unphysical propagating modes.

The action (1.14) can be supplemented by a matter action, containing a large number, $O(N)$, of free matter fields. One can then keep the product $N \cdot c_{d} G_{N}$ fixed, retain the usual normalization of the matter kinetic terms, and expand in powers of $1 / N$. Renormalizability of the resulting 'large $\mathrm{N}$ expansion' then amounts to being able to remove the UV cutoff order by order in the formal series in $1 / N$. This type of studies was initiated by Tomboulis where the gravity action was taken either the pure Ricci scalar [145], Ricci plus cosmological term [135], or a higher derivative action [146], with free fermionic matter in all cases. More recently the technique was reconsidered [120] with (1.14) as the gravity action and free matter consisting of $N n_{S}$ scalar fields, $N n_{D}$ Dirac fields, and $N n_{M}$ Maxwell fields.

Starting from the Einstein-Hilbert action the high energy behavior of the usual $1 / p^{2}$ type propagator gets modified. To leading order in $1 / N$ the modified propagator can be viewed as the graviton propagator with an infinite number of matter selfenergy bubbles inserted and resummed. The resummation changes the high momentum behavior from $1 / p^{2}$ to $1 /\left(p^{4} \ln p^{2}\right)$, in four dimensions. In $2<d<4$ dimensions the resulting $1 / N$ expansion is believed to be renormalizable in the sense that the UV cutoff $\Lambda$ can strictly be removed order by order in $1 / N$ without additional (counter) terms in the Lagrangian. In $d=4$ the same is presumed to hold provided an extra $C^{2}$ term is included in the bare Lagrangian, as in (1.14). After removal of the cutoff the beta functions of the dimensionless couplings can be analyzed in the usual way and already their leading $1 / N$ term will decide about the flow pattern.

The qualitative result (due to $[145,135]$ ) is that there exists a nontrivial fixed point for the dimensionless couplings $\mathrm{g}_{N}, \lambda$, and $s$. Its unstable manifold is three dimensional, i.e. all couplings are asymptotically safe. Repeating the computation in $2+\epsilon$ dimensions the fixed point still exists and (taking into account the different UV regularization) corresponds to the large $c$ (central charge) limit of the fixed point found the $2+\epsilon$ expansion.

These results have recently been confirmed and extended by Percacci [120] using the heat kernel expansion. In the presence of $N n_{S}$ scalar fields, $N n_{D}$ Dirac fields, and $N n_{M}$ 
Maxwell fields, the flow equations for $\mathrm{g}_{N}, \lambda$ and $s$ come out to leading order in $1 / N$ as

$$
\begin{aligned}
\mu \frac{d}{d \mu} \mathrm{g}_{N} & =2 \mathrm{~g}_{N}+\frac{1}{(4 \pi)^{2}} \frac{1}{6}\left(n_{S}-2 n_{D}-4 n_{M}\right) \mathrm{g}_{N}^{2} \\
\mu \frac{d}{d \mu} \lambda & =-2 \lambda+\frac{1}{(4 \pi)^{2}}\left[\frac{1}{6}\left(n_{S}-2 n_{D}-4 n_{M}\right) \lambda \mathrm{g}_{N}+\frac{1}{4}\left(n_{S}-4 n_{D}+2 n_{M}\right) \mathrm{g}_{N}\right] \\
\mu \frac{d}{d \mu} s & =-\frac{1}{(4 \pi)^{2}} \frac{1}{360}\left(6 n_{S}+25 n_{D}+72 n_{M}\right) s^{2}
\end{aligned}
$$

One sees that the $C^{2}$ coupling is always asymptotically free, and that Newton's constant has a nontrivial fixed point, $\mathrm{g}_{N}^{*} /(4 \pi)^{2}=12 /\left(-n_{S}+2 n_{D}+4 n_{M}\right)$, which is positive if the number of scalar matter fields is not too large.

As a caveat one should add that the $1 / p^{4}$-type propagators occuring both in the perturbative and in the large $N$ framework are bound to have an unphysical pole at some intermediate momentum scale. This pole corresponds to unphysical propagating modes and it is the price to pay for (strict) perturbative renormalizability combined with asymptotically safe couplings. From this point of view, the main challenge of Quantum Gravidynamics lies in reconciling asymptotically safe couplings with the absence of unphysical propagating modes. This can be achieved in the context of the $2+2$ reduction.

(c) Evidence from symmetry reductions: Here one considers the usual gravitational functional integral but restricts it from "4-geometries modulo diffeomorphisms" to "4-geometries constant along a $2+2$ foliation modulo diffeomorphisms". This means instead of the familiar $3+1$ foliation of geometries one considers a foliation in terms of two-dimensional hypersurfaces $\Sigma$ and performs the functional integral only over configurations that are constant as one moves along the stack of two-surfaces. Technically this constancy condition is formulated in terms of two commuting vectors fields $K_{a}=K_{a}^{\alpha} \partial_{\alpha}$, $a=1,2$, that are Killing vectors of the class of geometries $q$ considered, $\mathcal{L}_{K_{a}} q_{\alpha \beta}=0$. For definiteness we consider here only the case where both Killing vectors are spacelike. From this pair of Killing vector fields one can form the symmetric $2 \times 2$ matrix $M_{a b}:=q_{\alpha \beta} K_{a}{ }^{\alpha} K_{b}{ }^{\beta}$. Then $\gamma_{\alpha \beta}:=q_{\alpha \beta}-M^{a b} K_{a \alpha} K_{b \beta}$ (with $M^{a b}$ the components of $M^{-1}$ and $\left.K_{a \alpha}:=q_{\alpha \beta} K_{a}^{\beta}\right)$ defines a metric on the orbit space $\Sigma$ which obeys $\mathcal{L}_{K_{a}} \gamma_{\alpha \beta}=0$ and $K_{a}^{\alpha} \gamma_{\alpha \beta}=0$. The functional integral is eventually performed over metrics of the form

$$
q_{\alpha \beta}=\gamma_{\alpha \beta}+M^{a b} K_{a \alpha} K_{b \beta}
$$

where the 10 components of a metric tensor are parameterized by the $3+3$ independent functions in $\gamma_{\alpha \beta}$ and $M_{a b}$. Each of these functions is constant along the stack of twosurfaces but may be arbitrarily rough within a two-surface.

In the context of the asymptotic safety scenario the restriction of the functional integral to metrics of the form (1.16) is a very fruitful one: (i) the restricted functional integral inherits the perturbative non-renormalizability (with finitely many relevant couplings) 
from the full theory. (ii) it takes into account the crucial 'spin-2' aspect, that is, linear and nonlinear gravitational waves with two independent polarizations per spacetime point are included. (iii) it goes beyond the Eikonal approximation [141, 49] whose dynamics can be understood via a related $2+2$ decomposition $[80,51]$. (iv) based on heuristic arguments the dynamics of full Quantum Gravidynamics is expected to be effectively two-dimensional in the extreme ultraviolet with qualitative properties resembling that of the $2+2$ truncation. The renormalization of the $2+2$ truncation can thus serve as a prototype study and its asymptotic safety probes the selfconsistency of the scenario. (v) for the restricted functional integral the full infinite dimensional renormalization group dynamics can be studied; it reveals both a Gaussian and a non-Gaussian fixed point, where the properties of the latter are compatible with the existence of a non-perturbative continuum limit. Two additional bonus features are: in this sector the explicit construction of Dirac observables is feasible (classically and presumably also in the quantum theory). Finally a large class of matter couplings is easily incorporated.

As mentioned the effective dynamics looks two-dimensional. Concretely the classical action describing the dynamics of the 2-Killing vector subsector is that of a non-compact symmetric space sigma-model non-minimally coupled to 2D gravity via the "area radius"

$\rho:=\sqrt{\operatorname{det}\left(M_{a b}\right)_{1 \leq a, b \leq 2}}$, of the two Killing vectors. To avoid a possible confusion let us stress, however, that the system is very different from most other models of quantum gravity (mini-superspace, 2D quantum gravity or dilaton gravity, Liouville theory, topological theories) in that it has infinitely many local and selfinteracting dynamical degrees of freedom. Moreover these are literally (an infinite subset of) the degrees of freedom of the 4-dimensional gravitational field, not just analogues thereof. The corresponding classical solutions (for both signatures of the Killing vectors) have been widely studied in the General Relativity literature, c.f. $[69,18,87]$. We refer to [35, 36] for details on the reduction procedure and [130] for a canonical formulation. The case with aligned polarizations (Beck-Einstein-Rosen waves) is much simpler and the essential aspects can be modelled by a massive free field on $\mathrm{AdS}_{2}$ [107].

For generic polarizations strongly selfinteracting systems arise whose the renormalization [108] can be achieved by borrowing covariant background field techniques from Riemannian sigma-models; see $[78,57,79,133,39,148,114]$. In the particular application here the sigma-model perturbation theory is partially nonperturbative from the viewpoint of a graviton loop expansion as not all of the metric degrees of freedom are Taylor expanded in the bare action, see [110]. This together with the field reparameterization invariance blurs the distinction between a perturbative and a non-perturbative treatment of the gravitational modes. The renormalization can be done to all orders of sigma-model perturbation theory, which is 'not-really-perturbative' for the gravitational modes. It turns out that strict cutoff independence can be achieved only by allowing for infinitely many essential couplings. They are conveniently combined into a generating functional $h$, which is a positive function of one real variable. Schematically the renormalized action 
takes the form [108]

$$
S[q]=S_{E H}\left[\frac{h(\rho)}{\rho} q\right]+\text { other second derivative terms }
$$

Here $q$ is a metric of the form (1.16), $S_{E H}[q]$ is the Einstein-Hilbert action evaluated on it, and $h(\rho)$ is the generating coupling function evaluated on the renormalized area radius field $\rho$. Higher derivative terms are not needed in this subsector for the absorption of counter terms; the "other second derivative terms" needed are known explicitly.

This "coupling functional" is scale dependent and is subject to a flow equation of the form

$$
\mu \frac{d}{d \mu} h=\boldsymbol{\beta}_{h}(h),
$$

where $\mu$ is the renormalization scale and $\mu \mapsto h(\cdot, \mu)$ is the 'running' generating functional. To preclude a misunderstanding let us stress that the function $h(\cdot, \mu)$ changes with $\mu$, irrespective of the name of the argument, not just its value on $\rho$, say. Interestingly a closed formula for the beta function (or functional) in (1.18) can be found [108, 109]. The resulting flow equation is a nonlinear partial integro-differential equation and difficult to analyze. The fixed points however are easily found. Apart from the degenerate 'Gaussian' one, $1 / h \equiv 0$, there is a nontrivial fixed point $h^{\text {beta }}$. For the Gaussian fixed point a linearized stability analysis is empty, the structure of the quadratic perturbation equation suggests that it has both attractive and repulsive directions in the space of functions $h$. For the non-Gaussian fixed point $h^{\text {beta }}$ a linearized stability analysis is non-empty and leads to a system of linear integro-differential equations. Since the fixed point $h^{\text {beta }}$ has the form of a powerseries in the loop counting parameter $\lambda$, the proper concept of a "linearized perturbation" has the form

$$
\begin{aligned}
h(\rho, \lambda, \mu) & =h^{\text {beta }}(\rho, \lambda)+\delta h(\rho, \lambda, \mu), \\
\delta h(\rho, \lambda, \mu) & =\frac{\lambda}{2 \pi} \mathrm{s}_{1}(\rho, t)+\left(\frac{\lambda}{2 \pi}\right)^{2} \mathrm{~s}_{2}(\rho, t)+\ldots
\end{aligned}
$$

where the $\mathrm{s}_{l}(\rho, t)$ are functions of $\rho$ and $t=\frac{1}{2 \pi} \ln \mu / \mu_{0}$. Note that the perturbation involves infinitely many functions of two variables. Inserting the ansatz (1.19) into the flow equation $\mu \frac{d}{d \mu} h=\boldsymbol{\beta}_{h}(h / \lambda)$ and linearizing in $\delta h(\rho, \lambda, \mu)$ gives a recursive system of inhomogeneous integro-differential equations for the $s_{l}$, The boundary conditions are fixed such that the full $h$ flow is driven by the counterterms only, which amounts to the requirement that all the $s_{l}(\rho, t)$ vanish for $\rho \rightarrow \infty$ uniformly in $t$. Subject to these boundary conditions the recursive system of integro-differential equations can be shown to have a unique solution for arbitary smooth initial data. The solution for $\mathrm{s}_{1}$ reads

$$
\mathrm{s}_{1}(\rho, t)=\rho \int_{\rho}^{\infty} \frac{d u}{u} r_{1}\left(u-\zeta_{1} t\right)
$$


where $r_{1}$ is an arbitrary smooth function of one variable satisfying $u r_{1}(u) \rightarrow 0$ for $u \rightarrow 0$. This function can essentially be identified with the initial datum at some renormalization time $t=0$, as $r_{1}(\rho)=-\rho \partial_{\rho}\left[\mathbf{s}_{1}(\rho, t=0) / \rho\right]$. Evidently $\mathrm{s}_{1}(\rho, t) \rightarrow 0$ for $t \rightarrow \infty$, if $\zeta_{1}<0$.

This condition is indeed satisfied by all the symmetry reduced gravity theories considered in [109], precisely because the coset space $G / H$ is noncompact. If sigma-model scalars and abelian gauge fields are present in the $4 \mathrm{D}$ action one has the simple formula

$$
\zeta_{1}=-\frac{k+2}{2}, \quad k=\# \text { abelian vector fields }
$$

Equation (1.20) shows that the lowest order perturbation $s_{1}$ will always die out for $t \rightarrow \infty$, for arbitrary smooth initial data prescribed at $t=0$. It can be shown that this continues to hold for all higher order $s_{l}$ irrespective of the signs of the coefficients $\zeta_{l}, l \geq 2$. The situation is illustrated in the Figure below. The proof of this result is somewhat technical and can be found in [109].

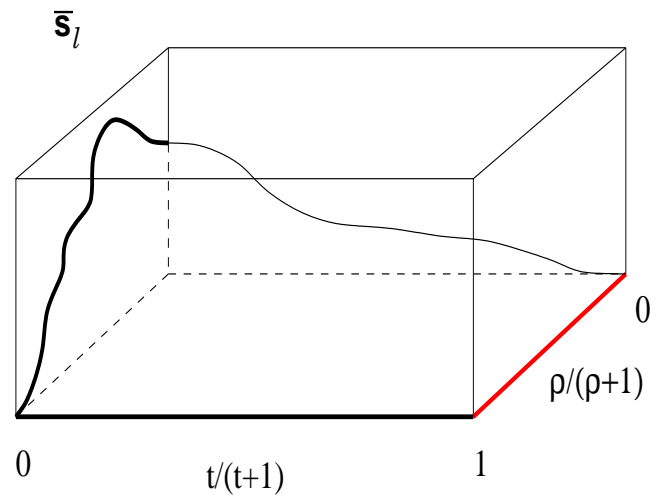

Thus all linearized perturbations decay for $\mu \rightarrow \infty$, which is precisely what Weinberg's criterion for asymptotic safety asks for. Moreover the basic propagator used is free from unphysical poles. This suggests that a genuine continuum limit exist for the $2+2$ reduced Quantum Gravidynamics beyond approximations (like the sigma-model perturbation theory/partially nonperturbative graviton expansion used to compute (1.18)). See [111, 89] for a proposed 'exact' bootstrap construction, whose relation to a $2+2$ truncated functional integral however remains to be understood.

In summary, in the context of the $2+2$ reduction an asymptotically safe coupling flow can be reconciled with the absence of unphysical propagating modes. In contrast to the technique on which evidence (d) below is based the existence of an infinite cutoff limit here can be shown and does not have to be stipulated as a hypothesis subsequently probed for selfconsistency. Since the properties of the $2+2$ truncation qualitatively are the ones one would expect from an 'effective' field theory describing the extreme UV aspects of Quantum Gravidynamics (see the end of Section 3), its asymptotic safety is a strong argument for the selfconsistency of the scenario. 
(d) Evidence from truncated flows of the effective average action: The effective average action $\Gamma_{\Lambda, k}$ for a scalar field theory is a generating functional generalizing the usual effective action, to which it reduces for $k=0$. Here $\Gamma_{\Lambda, k}$ depends on the UV cutoff $\Lambda$ and an additional scale $k$, indicating that in the defining functional integral roughly the field modes with momenta $p$ in the range $k \leq p \leq \Lambda$ have been integrated out. Correspondingly $\Gamma_{\Lambda, \Lambda}$ gives back the bare action and $\Gamma_{\Lambda, 0}=\Gamma_{\Lambda}$ is the usual quantum effective action, in the presence of the UV cutoff $\Lambda$. The modes in the momentum range $k \leq p \leq \Lambda$ are omitted or suppressed by a mode cutoff 'action' $C_{\Lambda, k}$, and one can think of $\Gamma_{\Lambda, k}$ as being the conventional effective action $\Gamma_{\Lambda}$ but computed with a bare action that differs from the original one by the addition of $C_{\Lambda, k}$; specifically

$$
\Gamma_{\Lambda, k}=-C_{\Lambda, k}+\left.\Gamma_{\Lambda}\right|_{S \mapsto S+C_{\Lambda, k}} .
$$

From the regularized functional integral defining $\Gamma_{\Lambda, k}$ an ('exact') functional renormalization group equation (FRGE) can be derived. Schematically it has the form $k \frac{d}{d k} \Gamma_{\Lambda, k}=$ rhs, where the "right hand side" involves the Hessian of $\Gamma_{\Lambda, k}$ with respect to the dynamical fields. The FRGE itself (that is, its rhs) carries no explicit dependence on the UV cutoff, or one which can trivially be removed. However the removal of the UV regulator $\Lambda$ implicit in the definition of $\Gamma_{\Lambda, k}$ is nontrivial and is related to the traditional UV renormalization problem. Whenever massless degrees of freedom are involved also the existence of the $k \rightarrow 0$ limit of $\Gamma_{\Lambda, k}$ is nontrivial and requires identification of the proper infrared degrees of freedom. In the present context we take this for granted and focus on the UV aspects.

The effective average action has been generalized to gravity by Reuter [127]. The substitution (1.22) is now applied to the (highly nonlocal) background effective action $\Gamma[g, \bar{g}]$ which in addition to the average $g$ of the 'quantum' metric $q$ depends on a background metric $\bar{g}$. The mode cutoff functional $C_{\Lambda, k}[q, \bar{g}]$ depends covariantly on $\bar{g}$ and the bare action $S_{\Lambda}[q, \bar{g}]=\Gamma_{\Lambda, \Lambda}[q, \bar{g}]$ is not specified from the outset. In fact, conceptually it is largely determined by the requirement that a continuum limit exists, see the criterion in Appendix A.2. $\Gamma_{\Lambda, \Lambda}$ can be expected to have a well-defined derivative expansion with the leading terms roughly of the form (1.14). Also the gravitational effective average action $\Gamma_{\Lambda, k}$ obeys an 'exact' FRGE, which is a new computational tool in quantum gravity not limited to perturbation theory. In practice $\Gamma_{\Lambda, k}$ is replaced in this equation with a $\Lambda$ independent functional interpreted as $\Gamma_{\infty, k}$. The assumption that the 'continuum limit' $\Gamma_{\infty, k}$ for the gravitational effective average action exists is of course what is at stake here. The strategy in the FRGE approach is to show that this assumption, although without a-priori justification, is consistent with the solutions of the flow equation $k \frac{d}{d k} \Gamma_{\infty, k}=\mathrm{rhs}$ (where right hand side now also refers to the Hessian of $\Gamma_{\infty, k}$ ). The structure of the solutions $\Gamma_{k}$ of this cut-off independent FRGE should be such that they can plausibly be identified with $\Gamma_{\infty, k}$. Presupposing the 'infrared safety' in the above sense, a necessary condition for this is that the $\operatorname{limits} \lim _{k \rightarrow \infty} \Gamma_{k}$ and $\lim _{k \rightarrow 0} \Gamma_{k}$ exist. Since $k \leq \Lambda$ the first limit probes whether $\Lambda$ can be made large; the second condition is needed to 
have all modes integrated out. In other words one asks for global existence of the $\Gamma_{k}$ flow obtained by solving the cut-off independent FRGE. Being a functional differential equation the cutoff independent FRGE requires an initial condition, i.e. the specification of a functional $\Gamma_{\text {initial }}$ which coincides with $\Gamma_{k}$ at some scale $k=k_{\text {initial }}$. The point is that only for very special 'fine tuned' initial functionals $\Gamma_{\text {initial }}$ will the associated solution of the cutoff independent FRGE exist globally. The existence of the $k \rightarrow \infty$ limit in this sense can be viewed as the counterpart of the UV renormalization problem, namely the determination of the unstable manifold associated with the fixed point $\lim _{k \rightarrow \infty} \Gamma_{k}$. We refer to Appendix A.2 for a more detailed discussion of this issue.

The full nonlinear functional differential equation is of course intractable. To make the FRGE computationally useful the space of functionals is truncated typically to a finite dimensional one of the form

$$
\Gamma_{k}[g, \bar{g}]=\sum_{i=0}^{N} \mathrm{~g}_{i}(k) k^{d_{i}} I_{i}[g]+\text { gauge fixing term },
$$

where the $I_{i}$ are 'well-chosen' - local and nonlocal - functionals of $g$, and $\bar{g}$ is identified with $g$ after functional differentiation. The $\mathrm{g}_{i}(k)$ are numerical parameters that carry the scale dependence. For $I_{i}$ 's obeying a non-redundancy condition, the $g_{i}$ play the role of essential couplings which have been normalized to have vanishing mass dimension by taking out a power $k^{d_{i}}$. Beyond perturbation theory unfortunately little is known about the type of nonlocal terms to expect in $\Gamma_{k}[g, \bar{g}]$, leaving the choice of such $I_{i}$ somewhat arbitrary. Conceptually the truncation implicitly replaces the full gravitational dynamics by one whose functional renormalization flow is confined to the subspace (1.23), similar to what happens in a hierarchical approximation.

The original FRGE then can be converted into a system of nonlinear ordinary differential equations for the couplings $g_{i}$. In the case of gravity the following ansatz has been made by Lauscher and Reuter [94, 95] (with Euclidean signature)

$$
I_{0}[g]=\int d x \sqrt{g}, \quad I_{1}[g]=-\int d x \sqrt{g} R(g), \quad I_{2}[g]=\int d x \sqrt{g} R(g)^{2},
$$

where $g=\left(g_{\alpha \beta}\right)_{1 \leq \alpha, \beta \leq 4}$ is the metric and $R(g)$ is the associated curvature scalar. The flow pattern $k \mapsto\left(\mathrm{g}_{0}(k), \mathrm{g}_{1}(k), \mathrm{g}_{2}(k)\right)$ displays a number of remarkable properties. Most importantly a non-Gaussian fixed point exists (first found in [136] based on [127] and corroborated in $[137,94,95,98,28])$. Within the truncation (1.24) a three-dimensional subset of initial data is attracted to the fixed point under the reversed flow

$$
\lim _{k \rightarrow \infty}\left(\mathrm{g}_{0}(k), \mathrm{g}_{1}(k), \mathrm{g}_{2}(k)\right)=\left(\mathrm{g}_{0}^{*}, \mathrm{~g}_{1}^{*}, \mathrm{~g}_{2}^{*}\right)
$$

where the fixed point couplings $\mathrm{g}_{i}^{*}, i=0,1,2$, are finite and positive and no blow-up occurs in the flow for large $k$. Again this adheres precisely to the asymptotic safety criterion. 
The flow equations for the $\mathrm{g}_{i}(k)$ 's depend on the choice of the mode cutoff function and on the choice of gauge fixing. In general they do not assume a transparent analytical form. An exception is when all but $I_{0}$ and $I_{1}$ are omitted from the truncation (so that only the Einstein-Hilbert terms remain) and an optimized mode cutoff is used in combination with a limiting version of the gauge fixing term [98]. In terms of the parameterization $\mathrm{g}_{0}=2 \lambda / \mathrm{g}_{N}$ and $\mathrm{g}_{1}=1 / \mathrm{g}_{N}$ used later on the flow equations then take the form

$$
\begin{aligned}
k \frac{d}{d k} \mathrm{~g}_{N} & =2 \mathrm{~g}_{N}+\frac{6 \mathrm{~g}_{N}^{2}}{\mathrm{~g}_{N}-6(4 \pi)^{2}(1-2 \lambda)^{2}}, \\
k \frac{d}{d k} \lambda & =-2 \lambda-\frac{\mathrm{g}_{N}}{2(4 \pi)^{2}}\left(1+2 \frac{3 \mathrm{~g}_{N}+12(4 \pi)^{2}(1-3 \lambda)}{2 \mathrm{~g}_{N}-12(4 \pi)^{2}(1-2 \lambda)^{2}}\right) .
\end{aligned}
$$

The above properties can then be verified analytically.

Some of the trajectories with initial data in the unstable manifold cannot be extended to $k \rightarrow 0$ due to (infrared) singularities. This problem is familiar from nongravitational theories and is presumably an artifact of the truncation. In the vicinity of the fixed point, on the other hand, all trajectories show remarkable robustness properties against modifications of the mode cutoff scheme which provide good reasons to believe that the structural aspects of the above results are not an artifact of the truncation used. The upshot is that there is a clear signal for asymptotic safety in the subsector (1.23), obtained via truncated functional renormalization flow equations.

The impact of matter has been studied by Percacci et al [50, 121, 122]. Minimally coupling free fields (bosons, fermions, or abelian gauge fields) one finds that the nonGaussian fixed point is robust, but the positivity of the fixed point couplings $g_{0}^{*}>0, g_{1}^{*}>$ 0 puts certain constraints on the allowed number of copies. When a selfinteracting scalar $\chi$ is coupled nonminmally via $-\sqrt{g}\left[\left(\kappa_{0}+\kappa_{2} \chi^{2}+\kappa_{4} \chi^{4}+\ldots\right) R(g)+\lambda_{0}+\lambda_{2} \chi^{2}+\right.$ $\left.\lambda_{4} \chi^{4}+\ldots+\partial \chi \partial \chi\right]$, one finds a fixed point $\kappa_{0}^{*}>0, \lambda_{0}^{*}>0$ (whose values are with matched normalizations the same as $\mathrm{g}_{1}^{*}, \mathrm{~g}_{0}^{*}$ in the pure gravity computation) while all selfcouplings vanish, $\kappa_{2}^{*}=\kappa_{4}^{*}=\ldots=0, \lambda_{2}^{*}=\lambda_{4}^{*}=\ldots=0$. In the vicinity of the fixed point a linearized stability analysis can be performed; the admixture with $\lambda_{0}$ and $\kappa_{0}$ then lifts the marginality of $\lambda_{4}$, which becomes marginally irrelevant $[121,122]$. The running of $\kappa_{0}$ and $\lambda_{0}$ is qualitatively unchanged as compared to pure gravity, indicating that the asymptotic safety property is robust also with respect to the inclusion of selfinteracting scalars.

This concludes our survey of the evidence for asymptotic safety. More details on the results (c) and (d) can be found in the review [110]. The perturbative identification of the non-Gaussian fixed point is detailed in section 2.2. The results (c) and (d) are genuinely surprising. With hindsight, the most natural explanation is to view them as manifestations of the asymptotic safety of the full dynamics with respect to a nontrivial fixed point. Tentatively (c) reflects a property of the full dynamics in the extreme ultraviolet via the dimensional reduction of the residual interactions. Since $\Gamma_{\Lambda, \Lambda}=S_{\Lambda}$ the origin of $(d)$ could be the match to the perturbatively visible non-Gaussian fixed 
point.

\subsection{Some working definitions}

Here we attempt working definitions for some of the key terms used before.

Quantum Gravidynamics: The term is coined in analogy to "Quantum Chromodynamics" indicating, first, that the theory is supposed to be defined not only as an effective field theory and, second, that the selfinteraction of the quantized gravitational field is predominantly antiscreening in the ultraviolet.

In contrast to "Quantum General Relativity" the microscopic action is allowed to be different from the Einstein-Hilbert action or a discretization thereof. Plausibly it should be still quasilocal, i.e. have a well-defined derivative expansion, and based on perturbatively renormalizable higher derivative theories one would expect it to contain at least quartic derivative terms. This means that also the number of physical propagating degrees of freedom (with respect to a background) may be different from the number entailed by the Einstein-Hilbert action. As with "Quantum General Relativity" we take the term "Gravidynamics" in a broad sense, allowing for any set of field variables (e.g. vielbein and spin connection, Sen-Ashtekar variables, Plebanski and BF type formulations, teleparallel etc.) that can be used to recast general relativity (see e.g. the review [118]). It is of course not assumed from the outset that the quantum gravidynamics based on the various set of field variables are necessarily equivalent.

Gaussian fixed point: A fixed point is called Gaussian if there exists a choice of field variables for which the fixed point action is quadratic in the fields and the functional measure is Gaussian. This includes the local case but also allows for nonlocal quadratic actions. The drawback of this definition is that the proper choice of field variables in which the measure reveals its Gaussian nature may be hard to find. (For example in the correlation functions of the spin field in the two-dimensional Ising model the underlying free fermionic theory is not visible.)

A non-Gaussian fixed point is simply one where no choice of fields can be found in which the measure becomes Gaussian. Unfortunately this, too, is not a very operational criterion.

Unstable manifold: The unstable manifold of a fixed point with respect to a coarse graining operation is the set of all points that can be reached along flow lines emanating from the fixed point, the so-called renormalized trajectories. Points on such a flow line correspond to perfect actions. The stable manifold is the set of points attracted to the fixed point in the direction of coarse graining.

Strict (weak) renormalizability: We call a field theory strictly (weakly) renormalizable with respect to a fixed point and a coarse graining operation if the dimension of its unstable manifold is finite (infinite). It is implied that if a field theory has this property with respect to one coarse graining operation it will have it with respect to many others 
("universality"). Strict or weak renormalizability is believed to be a sufficient condition for the existence of a genuine continuum limit for observables.

Relevant coupling: Given an expansion "sum over couplings times interaction monomials", a coarse graining operation, and a fixed point of it, a coupling is called relevant (irrelevant) if it is driven away from (towards) the value the corresponding coordinate has at the fixed point, under a sufficient number of coarse graining steps. Note that this distinction makes sense even for trajectories not connected to the fixed point (because they terminate). It is however an explicitly 'coordinate dependent' notion. The same terms are used for the interaction monomials associated with the couplings. The dimension of the unstable manifold equals the maximal number of independent relevant interaction monomials 'connected' to the fixed point. All points on the unstable manifold are thus parameterized by relevant couplings but not vice versa.

Couplings which are relevant or irrelevant in a linearized analysis are called linearly relevant or linearly irrelevant, respectively. A coupling which is neither linearly relevant nor linearly irrelevant, is called (linearly) marginal.

Continuum limit: By a genuine continuum limit we mean here a limit in which physical quantities become: (C1) strictly independent of the UV cutoff, (C2) independent of the choice of the coarse graining operation (within a certain class), and (C3) independent of the choice of gauge slice and invariant under point transformations of the fields. Usually one stipulates properties $(\mathrm{C} 1)$ and $(\mathrm{C} 2)$ for the functional measure after which (C3) should be a provable property of physical quantities like the S-matrix. The requirement of having also (C1) and (C2) only for observables is somewhat weaker and in the spirit of the asymptotic safety scenario. For the issue of gauge-independence see [93, 117].

Typically the properties (C1-C3) cannot be rigorously established, but there are useful criteria which render the existence of a genuine continuum limit plausible in different computational frameworks. In Appendices A1 and A2 we discuss in some detail such criteria for the perturbative and for the FRGE approach, respectively. For convenience we summarize the main points here.

In renormalized perturbation theory the criterion involves two parts: (PTC1) Existence of a formal continuum limit. This means, the removal of the UV cutoff is possible and the renormalized physical quantities are independent of the scheme and of the choice of interpolating fields - all termwise in a formal power series in the loop counting parameter. The perturbative beta functions always have a have a trivial (Gaussian) fixed-point but may also have a nontrivial (non-Gaussian) fixed point. The second part of the criterion is: (PTC2) The dimension of the unstable manifold of the (Gaussian or non-Gaussian) fixed point as computed from the perturbative beta functions equals the number of independent essential couplings. For example $\phi_{4}^{4}$ and QED meet (PTC1) but not (PTC2) while QCD satisfies both (PTC1) and (PTC2).

In the framework of the functional renormalization group equations (FRGE) similar criteria for the existence of a genuine continuum limit can be formulated. Specifically 
for the FRGE of the effective average action one has: (FRGC1) The solution of the FRG equation admits (for fine tuned initial data $\Gamma_{\text {initial }}$ at some $k=k_{\text {initial }}$ ) a global solution $\Gamma_{k}$, i.e. one that can be extended both to $k \rightarrow \infty$ and to $k \rightarrow 0$ (where the latter limit is not part of the UV problem in itself). (FRGC2) The functional derivatives of $\lim _{k \rightarrow 0} \Gamma_{k}$ (vertex functions) meet certain requirements which ensure stability/positivity/unitarity.

In (FRGE1) the existence of the $k \rightarrow 0$ limit in theories with massless degrees of freedom is nontrivial and the problem of gaining computational control over the infrared physics should be separated from the UV aspects of the continuum limit as much as possible. However the $k \rightarrow 0$ limit is essential to probe stability/positivity/unitarity. For example, to obtain a (massive) Euclidean quantum field theory the Schwinger functions constructed from the vertex functions have to obey nonlinear relations which ensure that the Hilbert space reconstructed via the Osterwalder-Schrader procedure has a positive definite inner product.

Perturbative (weak) renormalizability: We call a theory perturbatively (weakly) renormalizable if (PTC1) can be achieved with finitely (infinitely) many essential couplings. A theory were neither can be achieved is called perturbatively nonrenormalizable. Perturbative (weak) renormalizability is neither necessary nor sufficient for (weak or strict) renormalizability in the above nonperturbative sense. It is only in combination with (PTC2) that perturbative results are indicative for the existence of a genuine continuum limit.

Asymptotically free coupling: A non-constant coupling in the unstable manifold of a Gaussian fixed point.

The "non-constant" proviso is needed to exclude cases like a trivial $\phi_{4}^{4}$ coupling. In a nonperturbative lattice construction of $\phi_{4}^{4}$ theory only a Gaussian fixed point with a one-dimensional unstable manifold (parameterized by the renormalized mass) is thought to exist, along which the renormalized $\phi_{4}^{4}$ coupling is constant and identically zero. The Gaussian nature of the fixed-point, on the other hand, is not crucial and we define:

Asymptotically safe coupling: A non-constant coupling in the unstable manifold of a fixed point.

Asymptoticaly safe functional measure: The functional measure of a statistical field theory is said to be asymptotically safe if it is perturbatively weakly renormalizable or non-renormalizable, but it possesses a fixed point with respect to which it is strictly renormalizable. Subject to the regularity assumption that the space of actions can in the vicinity of the fixed point be decomposed into a stable and an unstable manifold this is equivalent to the following requirement: all relevant couplings are asymptotically safe and there is only a finite number of them. Note that unitarity or other desirable properties that would manifest itself on the level of observables are not part of this definition.

In a non-gravitational context the functional measure of the 3D Gross-Neveu model is presently the best candidate to be asymptotically safe in the above sense (see [72, 41, 131, 
76] and references therein). Also 5D Yang-Mills theories (see [65, 104] and references therein) are believed to provide examples. In a gravitational context, however, there are good reasons to modify this definition.

First the choice of couplings has to be physically motivated, which requires to make contact to observables. In the above nongravitational examples with a single coupling the 'meaning' of the coupling is obvious; in particular it is clear that it must be finite and positive at the non-Gaussian fixed point. In general however one does not know whether ill behaved couplings are perverse redefinitions of better behaved ones. To avoid this problem the couplings should be defined as coefficients in a power series expansion of the observables themselves (Weinberg's "reaction rates", see the discussion in section 1.1). Of course painfully little is known about (generic) quantum gravity observables, but as a matter of principle this is how couplings should be defined. In particular this will pin down the physically aedequate notion of positivity or unitarity.

Second, there may be good reasons to work initially with infinitely many essential couplings. Recall that the number of essential couplings entering the initial construction of the functional measure is not necessarily equal to the number eventually indispensable. In a secondary step a reduction of couplings might be feasible. That is, relations among the couplings might exist which are compatible with the renormalization flow. If these relations are sufficiently complicated, it might be better to impose them retroactively than to try to switch to a more adapted basis of interaction monomials from the beginning.

Specifically in the context of quantum gravity microscopic actions with infinitely many essential couplings occur naturally in several ways: when starting from the Gomis and Weinberg picture [66] of perturbative quantum gravity and in the $2+2$ reduction [108], where a coupling function is needed for a dimensionless scalar. Further, the (Wilsonian) effective actions induced by the conformal anomaly can be rewritten in terms of dimensionless scalars $[16,10]$. Their functional form is only partially constrained by the requirement to reproduce the anomaly and the fate of the associated couplings or coupling functions in the ultraviolet is in principle a matter of dynamics.

Third, the dimension of the unstable manifold is of secondary importance in this context. Recall that the dimension of the unstable manifold is the maximal number of independent relevant interaction monomials 'connected' to the fixed point. This maximal number may be difficult to determine in Quantum Gravidynamics for the above reasons. Moreover the identification of all renormalized trajectories emanating from the fixed point may be more than what is needed physicswise; the successful construction of a subset of renormalized trajectories for physically motivated couplings may already be enough to obtain predictions/explanations for some observables. What matters is not so much the total number of relevant couplings but the way how observables depend on them. We remark that even in conventional perturbation theory based on the EinsteinHilbert action the divergencies in the S-matrix seem to be less severe than those in the effective action [23]. Generally, since generic observables (in the sense used in Section 
1.1) are likely to be nonlinearly and nonlocally related to the metric or to the usual basis of interaction monomials (scalars built from polynomials in the curvature tensors, for instance) the condition that the theory should allow for predictions in terms of observables is only indirectly related to the total number of relevant couplings.

In summary, the interplay between the microscopic action, its parameterization through essential or relevant couplings, and observables is considerably more subtle than in the presumed non-gravitational examples of asymptotically safe theories with a single coupling. The existence of an asymptotically safe functional measure in the above sense seems to be neither necessary nor sufficient for a physically viable theory of Quantum Gravidynamics. This leads to our final working definition.

Asymptotically safe Quantum Gravidynamics: A quantum theory of gravity based on a notion of an asymptotically safe functional integral measure which incorporates the interplay between couplings and observables described above. In brief: (i) the choice of couplings has to be based on observables; this will pin down the physically relevant notion of positivity/unitarity. (ii) the number of essential or relevant couplings is not a-priori finite. (iii) what matters is not so much the dimension of the unstable manifold than how observables depend on the relevant couplings.

\subsection{Discussion of possible objections}

Here we discuss some of the possible objections to a physically viable theory of Quantum Gravidynamics.

Q1 Since the microscopic action is likely to contain higher derivative terms don't the problems with non-unitarity notorious in higher derivative gravity theories reappear?

A1 In brief, the unitarity issue has not much been investigated so far, but the presumed answer is No.

First, the problems with perturbatively strictly renormalizable higher derivative theories stem mostly from the $1 / p^{4}$-type propagator used. The alternative perturbative framework already mentioned, namely to use a $1 / p^{2}$-type propagator at the expense of infinitely many essential (potentially 'unsafe') couplings avoids this problem $[66,8]$. The example of the $2+2$ reduction shows that the reconcilation of safe couplings with the absence of unphysical propagating modes can be achieved in principle. Also the superrenormalizable gravity theories with unitary propagators proposed in [144] are intriguing in this respect.

Second, even for higher derivative theories on flat space a well-defined Euclidean functional integral can exist, free of negative norm states or negative probabilities [75]. Physical unitarity is then thought to be restored at low energies, in which 
case one could 'live with' higher derivative ghosts. The same would presumably hold for higher derivative theories on a fixed curved background.

Third, when the background effective action is used as the central object to define the quantum theory, the 'background' is not a solution of the classical field equations. Rather it is adjusted selfconsistenly by a condition involving the full quantum effective action. If the background effective action is computed nonperturbatively (by whatever technique) the intrinsic notion of unitarity will not be related to the 'propagator unitarity' around a solution of the classical field equations in any simple way.

One aspect of this intrinsic positivity is the convexity of the background effective action. In the flow equation for the effective average action one can see, for example, that the wrong-sign of the propagator is not an issue: if $\Gamma_{k}$ is of the $R+R^{2}$ type, the running inverse propagator $\Gamma_{k}^{(2)}$ when expanded around flat space has ghosts similar to those in perturbation theory. For the $\Gamma_{k}$ flow, however, this is irrelevant since in the derivation of the beta functions no background needs to be specified explicitly. All one needs is that the RG trajectories are well defined down to $k=0$. This requires that $\Gamma_{k}^{(2)}+\mathcal{R}_{k}$ is a positive operator for all $k$. In the untruncated functional flow this is believed to be the case. A rather encouraging first result in this direction comes from the $R^{2}$ truncation [95].

More generally, the reservations towards higher derivative theories came from a loop expansion around flat space and near the perturbative Gaussian fixed point. In contrast in Quantum Gravidynamics one aims at constructing the continuum limit nonperturbatively at a different fixed point and with respect to a dynamically adjusted background. The status of unitarity and causality have then not even been explored in toy models.

In the previous discussion we implicitly assumed that generic physical quantities are related in a rather simple way to the interaction monomials entering the microscopic action. For Dirac observables however this is clearly not the case. Assuming that the physically correct notion of unitarity concerns such observables it is clear that the final word on unitarity issues can only be spoken once actual observables are understood.

Q2 Doesn't the very notion of renormalizability presuppose a length or momentum scale referring to a prescribed background spacetime?

A2 In some sense a coarse graining procedure is a 'background structure', not intrinsic to generic physical quantities, which is as inevitable as it is innocuous. However a fixed background spacetime is not needed in principle. As sketched in the intoduction it is part of the physics premise of a functional integral based approach that there is a physically relevant distinction between coarse grained and fine grained geometries. Geometrically motivated and tested proposals for coarse 
graining operations are presently not available, but there is certainly no obstruction of principle. Since the background field formalism is well-tested one may for the time being define the coarse graining with respect to a dynamically adjusted background metric, as described in section 1.2.

Q3 Doesn't such a non-perturbative renormalizability scenario require a hidden enhanced symmetry?

A3 Improved renormalizability properties around a given fixed point are indeed often rooted in symmetries. A good example is QCD in a lightfront formulation where gauge invariance is an 'emergent phenomenon' occuring only after an infinite reduction of couplings [123]. In the case of Quantum Gravidynamics, the symmetry in question would be one that becomes visible only around the non-Gaussian fixed point. If it exists, its identification would constitute a breakthrough. From the Kadanoff-Wilson view of renormalization it is however the fixed point which is fundamental - the enhanced symmetry properties are a consequence (see the notion of generalized symmetries in $[159,113]$.

Q4 Shouldn't the proposed anti-screening be seen in perturbation theory?

A4 Maybe maybe not. Presently no good criterion for antiscreening in this context is known. For the reasons explained in section 1.1 it should not merely be identified with the sign of the dominant contribution to some beta function. The answer will thus depend somewhat on the identification of the proper degrees of freedom and the quantity considered.

In the literature quantum gravity corrections to the Newton potential have been considered in some detail. The result is always of the form

$$
V(r)=-\frac{G m_{1} m_{2}}{r}\left[1+3 \frac{G\left(m_{1}+m_{2}\right)}{r}+\zeta \frac{\hbar G}{r^{2}}\right] .
$$

Interpreted as a modification of Newton's constant $G(r)=G\left(1+\zeta \hbar G / r^{2}\right)$, one sees that $\zeta>0$ roughly corresponds to screening and $\zeta<0$ to anti-screening behavior. The value of $\zeta$ is unambigously defined in 1-loop perturbation theory and is a genuine prediction of quantum gravity viewed as an effective field theory (as stressed by Donoghue). However $\zeta$ will depend on the precise definition of the nonrelativistic potential and there are various options for it.

One is via the $2 \rightarrow 2$ scattering amplitude. The coefficient $\zeta_{\text {scatt }}$ was computed initially by Donoghue and later by Khriplovich-Kirilin; the result considered definite in [27] is $\zeta_{\text {scatt }}=\frac{41}{10 \pi}$. It decomposes into a negative vertex and triangle contributions $\zeta_{v}=-\frac{105}{3 \pi}$, and a just slightly larger positive remainder $\zeta_{\text {scatt }}-\zeta_{v}=\frac{117.3}{3 \pi}$ coming from box, seagull, and vacuum polarization diagrams. 
Another option is to consider corrections to the Schwarzschild metric. Different sets of diagrams have been used for the definition $[86,26]$ and affect the reparameterization (in-)dependence and other properties of the corrections. Both choices advocated lead to $\zeta_{\text {metric }}<0$, which amounts to anti-screening.

Let us also mention alternative definitions of an effective Newton potential via Wilson lines in Regge calculus [71] or by resummation of scalar matter loops [149]. The latter gives rise to an "antiscreening" Yukawa type correction of the form $V(r)=-\frac{G}{r}\left(1-e^{-r / \sqrt{\zeta G}}\right)$, with $\zeta>0$. Via $V(r)=\int \frac{d^{3} k}{(2 \pi)^{3}} e^{i \vec{k} \cdot \vec{x}} G(k) / \vec{k}^{2}$ it can be interpreted as a running Newton constant $G(k)=G /\left(1+\zeta \vec{k}^{2}\right)$. Finally, in higher derivative theories of the form (1.14) Yukawa type corrections already occurs at tree level, e.g. $V(r)=-\frac{G}{r}\left(1-\frac{4}{3} e^{-r \sqrt{s /(16 \pi G)}}\right)$, for $\omega=\theta=0$ [139].

Q5 There are several thought experiments suggesting a fundamental limit to giving an operational meaning to spacetime resolutions, for example via generalized uncertainty relations of the form $([59,115,100]$ and references therein)

$$
\Delta x \approx \frac{\hbar}{\Delta p}+\frac{G \hbar}{c^{3}} \frac{\Delta p}{\hbar} .
$$

These relations are sometimes taken as hinting at a "fundamental discretum". If so, doesn't this contradict the asymptotic safety scenario, where in the fixed point regime the microscopic spacetimes become selfsimilar?

A5 No, the arguments assume that Newton's constant $G$ is constant. (We momentarily write $G$ for $G_{N}$ in $\left(16 \pi G_{N}\right)^{-1} \int d x \sqrt{g} R(g)$.) If $G$ is treated as a running coupling the derivations of the uncertainty relations break down. As an example consider a photon-electron scattering process as in [100,115]: $G$ refers to gravity in the ('photon' $k$ - 'electron' $\Delta p$ ) interaction region with a pointlike 'electron'. If viewed as running one expects $G(k) \approx G_{*} / k^{2}$ in the fixed point regime. Hence in the above relation one should replace $G$ by $\frac{G_{*} c^{3}}{(\Delta p)^{2}}$. This gives

$$
\Delta x \Delta p \approx \hbar\left(1+G_{*}\right)
$$

and there is no limit on the spatial resolution. One can of course decide to choose units in which $G$ is constant by definition (see [119]) in which case the derivations go through. Our conclusion is that the perceived dichotomy between a fundamentally 'discrete' versus 'continuum' geometry may itself not be fundamental.

Each of the issues raised clearly deserves much further investigation. For the time being we conclude however that the asymptotic safety scenario is conceptually selfconsistent. 


\section{The role of perturbation theory}

The application of renormalization group ideas to quantum gravity has a long history. In the following we focus on the role of perturbation theory for two reasons. First, because arguably the best non-perturbative implementation of a gravitational renormalization group still remains to found. Second, because nonperturbative techniques are typically ill-adapted to make definite statements about the UV regime - the regime under scrutiny here. In contrast, UV renormalized perturbation theory (PT), provided it is indicative for the behavior of an underlying 'exact theory' at all, is tailor-made for the investigation of the UV regime.

Technically the key advantage of PT is that the UV cutoff can strictly be removed, termwise to all loop orders in a perturbatively (strictly or weakly) renormalizable field theory. The price to pay for this advantage is that the series is at best asymptotic to the (unknown) exact answer supposed to exist. Indeed, whether or not an underlying 'exact theory' exists can then only be assessed in terms of a plausibility criterion involving the perturbative beta functions of the theory; see Appendix A for a discussion.

Despite the perturbative nonrenormalizability of the Einstein-Hilbert action there are two instructive ways in which strict or weak renormalizability can be achieved perturbatively in higher derivative theories. By higher derivative theories we mean here gravitational theories whose bare action contains, in addition to the Einstein-Hilbert term, scalars built from powers of the Riemann tensor and its covariant derivatives. In overview there are two distinct perturbative treatments of such theories, which will be surveyed in sections 2.2 and 2.3 , respectively.

The first one, initiated by Stelle [138], uses $1 / p^{4}$ type propagators (in four dimensions) in which case a higher derivative action containing all (three) quartic derivative terms can be expected to be power counting renormalizable. In this case strict renormalizability with only 4 (or 5, if Newton's constant is included) couplings can be achieved [138]. However the $1 / p^{4}$ type propagators are problematic from the point of view of unitarity.

An alternative perturbative treatment of higher derivative theories was first advocated by Gomis and Weinberg [66]. The idea is try to maintain a $1 / p^{2}$ type propagator and include all (infinitely many) counterterms generated in the bare action. Consistency requires that quadratic counterterms (those which contribute to the propagator) can be absorbed by field redefinitions. As verified in [8] this is the case either in the absence of a cosmological constant term or when the background spacetime admits a metric with constant curvature.

\subsection{Does Newton's constant run?}

Before turning to renormalization aspects proper, let us describe the special role of Newton's constant in a diffeomorphism invariant theory with a dynamical metric. Let 
$S[g$, matter $]$ be any local action, where $g=\left(g_{\alpha \beta}\right)_{1 \leq \alpha, \beta \leq d}$ is the metric and the "matter" fields are not scaled when the metric is. Scale changes in the metric then give rise to a variation of the Lagrangian which vanishes on shell:

$$
\left.\frac{d}{d \omega^{2}} S\left[\omega^{2} g, \text { matter }\right]\right|_{\omega=1}=\int d x \sqrt{g} g_{\alpha \beta} \frac{\delta S[g, \text { matter }]}{\delta g_{\alpha \beta}}
$$

As a consequence one of the coupling parameters which in the absence of gravity would be essential (i.e. a genuine coupling) becomes inessential (i.e. can be changed at will by a redefinition of the fields). The running of this parameter, like that of a wave function renormalization constant, has no direct significance. If the pure gravity part contains the usual Ricci scalar term $Z_{N} \sqrt{g} R(g)$ the parameter that becomes inessential may be taken as its prefactor, i.e. may be identified with the inverse of Newton's constant, via

$$
Z_{N}^{-1}=2 \frac{d-2}{d-3} \operatorname{Vol}\left(S^{d-2}\right) G_{\text {Newton }}=: c_{d} G_{\text {Newton }}
$$

The normalization factor $c_{d}, d \geq 4$ [129], is chosen such that the coefficient in the nonrelativistic force law, as computed from $Z_{N} \sqrt{g} R(g)+L_{\text {matter }}$, equals $G_{\text {Newton }} \operatorname{Vol}\left(S^{d-2}\right)$. For $d=2,3$ a different normalization has to be adopted.

The physics interpretation of the inessential parameter $\omega$ is that it sets the absolute momentum or spectral scale. To see this we can think of $g_{\alpha \beta}$ as a reference metric in the background field formalism. For example for the spectral values $\nu(g)$ of the covariant Laplacian $\Delta_{g}$ associated with $g_{\alpha \beta}$ one has

$$
\nu\left(\omega^{2} g\right)=\omega^{-2} \nu(g)
$$

since $\Delta_{\omega^{2} g}=\omega^{-2} \Delta_{g}$. The spectral values play the role of a covariant momentum squared. Indeed, if the metric is taken dimensionless $\nu(g)$ carries dimension 2 (since $\Delta_{g}$ does) and for a flat metric $g_{\alpha \beta}=\eta_{\alpha \beta}$ they reduce to $-\nu(\eta)=k^{2}$, for plane waves labeled by $k$. From (2.3) one sees that rescaling of the metric and rescaling of the spectral values amout to the same thing. Since the former parameter is inessential the latter is too. Hence in a theory with a dynamical metric the three (conceptually distinct) inessential parameters: overall scale of the metric $\omega$, the inverse of Newton's constant $Z_{N}^{-1}=c_{d} G_{\text {Newton }}$, and the overall normalization of the spectral/momentum values are in one-to-one correspondence. For definiteness we take Newton's constant as the variant under consideration.

Being inessential the quantum field theoretical running of $G_{\text {Newton }}$ has significance only relative to the running coefficient of some reference operator. The most commonly used choice is a cosmological constant term $\tilde{\Lambda} \int d x \sqrt{g}$. Indeed

$$
G_{\text {Newton }} \tilde{\Lambda}^{\frac{d-2}{d}}=\text { const } \tau(\mu)^{2 / d}
$$


is dimensionless and invariant under constant rescalings of the metric [83]. One usually switches to dimensionless parameters via

$$
c_{d} G_{\text {Newton }}=\mu^{2-d} \mathrm{~g}_{N}(\mu), \quad \tilde{\Lambda}=2 \mu^{d} \frac{\lambda(\mu)}{\mathrm{g}_{N}(\mu)},
$$

where $\mu$ is some dimension one parameter which will be taken as 'renormalization group time'. The Einstein-Hilbert action then reads

$$
\frac{\mu^{d-2}}{\mathrm{~g}_{N}(\mu)} \int d x \sqrt{g}\left[R(g)-2 \mu^{2} \lambda(\mu)\right]
$$

Being dimensionless one expects the running of $\mathrm{g}_{N}(\mu)$ and $\lambda(\mu)$ to be governed by flow equations without explicit $\mu$ dependence

$$
\mu \frac{\partial}{\partial \mu} \mathrm{g}_{N}=\gamma_{\mathrm{g}}\left(\mathrm{g}_{N}, \lambda\right), \quad \mu \frac{\partial}{\partial \mu} \lambda=\beta_{\lambda}\left(\mathrm{g}_{N}, \lambda\right)
$$

For the essential parameter $\tau(\mu)=\mathrm{g}_{N}(\mu) \lambda(\mu)^{(d-2) / 2}$ obtained from (2.4) this gives

$$
\mu \frac{\partial}{\partial \mu} \tau=\tau\left[\frac{\gamma_{\mathrm{g}}}{\mathrm{g}_{\mathrm{N}}}+\frac{d-2}{2} \frac{\beta_{\lambda}}{\lambda}\right]
$$

In the present context we assume $\tau$ to be asymptotically safe, i.e.

$$
\sup _{\mu_{0} \leq \mu \leq \infty} \tau(\mu)<\infty, \quad \lim _{\mu \rightarrow \infty} \tau(\mu)=\tau_{*}<\infty
$$

where here $0<\tau_{*}<\infty$. Given (2.9) there are two possibilities. First, the various scheme choices are such that the parameters $\mathrm{g}_{N}(\mu)$ and $\lambda(\mu)$ are both nonsingular and approach finite values $g_{*}$ and $\lambda_{*}$ for $\mu \rightarrow \infty$. Second, the scheme choices are such that one of them becomes singular and the other vanishes for $\mu \rightarrow \infty$. Usually the first possibility is chosen; then the $\mathrm{g}_{N}(\mu)$ flow defined by the first equation in (2.7) has all the properties required for an essential asymptotically safe coupling. This 'nonsingular parametric representation' of the $\tau(\mu)$ coupling flow is advantageous for most purposes.

The second possibility is realized when inserting a singular solution of the equation for $\mathrm{g}_{N}(\mu)$ into the equation for $\lambda(\mu)$. This naturally occurs when working in Planck units. One makes use of the fact that an inessential parameter can be frozen at a prescribed value. Specifically fixing

$$
\left[G_{\text {Newton }}\right]^{\frac{1}{d-2}}=M_{\mathrm{Pl}} \approx 1.4 \times 10^{19} \mathrm{GeV}
$$


amounts to working with Planck units [119]. From (2.5) it then follows that

$$
\mathrm{g}_{N}(\mu)=c_{d}\left(\frac{\mu}{M_{\mathrm{Pl}}}\right)^{d-2}, \quad(d-2) c_{d}\left(\frac{\mu}{M_{\mathrm{Pl}}}\right)^{d-2}=\gamma_{g}(\mathrm{~g}, \lambda)
$$

We may assume that the second equation has a local solution $\mathrm{g}_{N}(\mu)=f\left(\lambda, \mu / M_{\mathrm{Pl}}\right)$. Reinserted into the $\lambda$ equation gives a flow equation

$$
\mu \frac{\partial}{\partial k} \lambda(\mu)=\widetilde{\beta}_{\lambda}\left(\lambda, \mu / M_{\mathrm{Pl}}\right)
$$

which now explicitly depends on $\mu$. Writing similarly $\tilde{\tau}_{*}:=\tau_{*}\left(f\left(\lambda, \mu / M_{\mathrm{Pl}}\right), \lambda\right)$ the condition defining the $\tau(\mu)$ fixed point becomes

$$
\left.\widetilde{\beta}_{\lambda}\right|_{\tilde{\tau}_{*}}=-2 \lambda
$$

Both formulations are mathematically equivalent to the extent the inversion formula $\mathrm{g}_{N}(\mu)=f\left(\lambda, \mu / M_{\mathrm{Pl}}\right)$ is globally defined. For definiteness we considered here the cosmological constant term as a reference operator, but the principle clearly generalizes.

\subsection{Is a non-Gaussian fixed point visible in PT?}

Here we present the perturbative treatment of higher derivative theories which renders them strictly renormalizable. Our proposed answer to the question raised is: Yes, in a setting which can be taken as indicative for the genuine renormalization flow. We write again $q_{\alpha \beta}$ for the metric entering the functional integral and use $(-,+, \ldots,+)$ signature metrics here.

Initially in $d=4+\epsilon$ dimensions we consider the general action containing up to four derivatives of the metric

$$
\begin{aligned}
S & =-\int d x \sqrt{q}\left[\tilde{\Lambda}-\frac{1}{c_{d} G_{N}} R+\frac{1}{2 s} C^{2}-\frac{\omega}{3 s} R^{2}+\frac{\theta}{s} E\right] \\
& =-\int d x \sqrt{q}\left[\frac{1}{c_{d} G_{N}}(2 \Lambda-R)+z R^{2}+y R_{\alpha \beta} R^{\alpha \beta}+x R_{\alpha \beta \gamma \delta} R^{\alpha \beta \gamma \delta}\right] .
\end{aligned}
$$

Here $C^{2}$ is the square of the Weyl tensor, $E$ is the integrand of the Gauss-Bonnet term, and a total derivative term, $\nabla^{2} R$, has been omitted. The parameterization of the coefficients by couplings $s, \omega, \theta$ is chosen for later convenience. The parameters in the second line are related to those in the first by $\Lambda=c_{d} G_{N} \tilde{\Lambda} / 2$ and

$$
s x=\frac{1}{2}+\theta, \quad s y=-\frac{2}{d-2}-4 \theta, \quad s z=-\frac{\omega}{3}+\theta+\frac{1}{(d-1)(d-2)} .
$$


In $d=4$ the Gauss-Bonnet term is negligible; however if dimensional regularization is used, $d \neq 4$, it must be kept. For $d=3$ both $E$ and $C^{2}$ vanish.

Expanding around flat space, $q_{\alpha \beta}=\eta_{\alpha \beta}+f_{\alpha \beta}$, the quadratic part of the action reads

$$
\begin{aligned}
S_{\tilde{\Lambda}=0}^{(2)}= & \int d^{4} x\left\{\frac{1}{4} \partial^{2} f^{\alpha \beta}\left(\frac{1}{c_{4} G_{N}}-\frac{1}{s} \partial^{2}\right)\left[P^{(2)} f\right]_{\alpha \beta}\right. \\
& \left.+\partial^{2} f^{\alpha \beta}\left(-\frac{1}{2 c_{4} G_{N}}+\frac{\omega}{s} \partial^{2}\right)\left[P^{(0)} f\right]_{\alpha \beta}\right\} .
\end{aligned}
$$

For simplicity we took $d=4$ here and omitted the cosmological constant term. Further $P^{(0)}, P^{(2)}$ are the projectors onto the spin 0,2 parts of $f_{\alpha \beta}$, i.e.

$$
P_{\alpha \beta, \gamma \delta}^{(0)}=\frac{1}{3} P_{\alpha \beta} P_{\gamma \delta}, \quad P_{\alpha \beta, \gamma \delta}^{(2)}=\frac{1}{2}\left[P_{\alpha \gamma} P_{\beta \delta}+P_{\alpha \delta} P_{\beta \gamma}\right]-\frac{1}{3} P_{\alpha \beta} P_{\gamma \delta},
$$

with $P_{\alpha \beta}=\eta_{\alpha \beta}-\partial^{-2} \partial_{\alpha} \partial_{\beta}$. One sees that the signs in (2.16) are such that for $s>0$ the spin two part gives rise to a positive definite Euclidean action. The corresponding part of the Euclidean propagator can be written as

$$
2 P_{\alpha \beta, \gamma \delta}\left(\frac{1}{p^{2}}-\frac{1}{p^{2}+s /\left(c_{4} G_{N}\right)}\right)
$$

and thus for fixed (bare) $s /\left(c_{4} G_{N}\right)$ displays the characteristic $+1 / p^{4}$ behavior. The spin zero part does not necessarily enter (2.16) with the 'good' sign, but it will be affected by gauge fixing and measure terms anyhow. See $[138,32]$ for a discussion.

The perturbative quantization of (2.14) proceeds as usual. Gauge fixing and ghost terms are added and the total action is expanded in powers of $f_{\alpha \beta}=q_{\alpha \beta}-\eta_{\alpha \beta}$. The one loop counter term (minus the divergent part of the effective action) has been computed by a number of authors [56, 15, 14, 24, 38], using different regularizations. The result of Avramidi and Barvinsky $[15,14]$ in dimensional regularization has been confirmed in [24] and in the present conventions reads

$$
\begin{aligned}
\Delta S^{(1)}= & -\Gamma_{\text {div }}^{(1)}=\frac{\mu^{d-4}}{(4 \pi)^{2}(d-4)} \int d^{d} x \sqrt{q}\left\{2 s(u(\omega)-\gamma(\omega)) \tilde{\Lambda}+\left(\frac{s}{c_{d} G_{N}}\right)^{2} \frac{1+20 \omega^{2}}{8 \omega^{2}}\right. \\
& \left.+\gamma(\omega) \frac{s}{c_{d} G_{N}} R+\frac{133}{20} C^{2}+\frac{5}{36}\left(1+12 \omega+8 \omega^{2}\right) R^{2}+\frac{196}{45} E\right\} .
\end{aligned}
$$

Here $u(\omega)=\left(-1+30 \omega+40 \omega^{2}\right) /(12 \omega)$ is independent of the gauge fixing parameters, while $\gamma(\omega)$ is gauge dependent. E.g. in harmonic gauge one has $\gamma(\omega)=\left(-1+10 \omega^{2}\right) /(3 \omega)$, while in the Vilkovisky-deWitt effective action $\gamma(\omega)=\left(-13+18 \omega+40 \omega^{2}\right) /(12 \omega)$ enters.

To absorb (2.19) singular field redefinitions should be taken into account. By inspection of the equations of motion operator for (2.14) one sees that only field redefinitions 
proportional to $q_{\alpha \beta}$ are of immediate use. We thus take

$$
q_{\alpha \beta}^{\mathrm{B}}=q_{\alpha \beta}+\frac{1}{(4 \pi)^{2}(d-4)} \xi q_{\alpha \beta}
$$

where $\xi$ can be a function of $s / \mathrm{g}_{N}, \lambda, \omega, \theta$. The 1-loop flow equations for $s, \omega, \theta$ obtained from (2.19) are universal

$$
\begin{aligned}
(4 \pi)^{2} \mu \frac{d}{d \mu} s & =-\frac{133}{10} s^{2} \\
(4 \pi)^{2} \mu \frac{d}{d \mu} \omega & =-\frac{25+1098 \omega+200 \omega^{2}}{60} s, \\
(4 \pi)^{2} \mu \frac{d}{d \mu} \theta & =\frac{7(56-171 \theta)}{90} s .
\end{aligned}
$$

These equations have a trivial fixed point $s_{*}=0, \omega_{*}=$ const, $\theta_{*}=$ const, and a nontrivial fixed point $s_{*}=0, \omega_{*}=-(549 \pm 7 \sqrt{6049}) / 200, \theta_{*}=56 / 171$. Importantly the $C^{2}$ coupling $s$ is asymptotically free.

To describe the flow of the Newton and cosmological constants one switches to the dimensionless parameters $g_{N}$ and $\lambda$ as in Section 2.1. From (2.19) and (2.20) one finds

$$
\begin{aligned}
\mu \frac{d}{d \mu} \mathrm{g}_{N} & =2 \mathrm{~g}_{N}+\frac{1}{(4 \pi)^{2}} \operatorname{sg}_{N}\left[\gamma(\omega)+\partial_{s} \xi\right] \\
\mu \frac{d}{d \mu} \lambda & =-2 \lambda+\frac{1}{(4 \pi)^{2}} s \lambda\left[2 u(\omega)-\gamma(\omega)-\partial_{s} \xi+\frac{s}{\mathrm{~g}_{N} \lambda} \frac{1+20 \omega^{2}}{8 \omega^{2}}\right] .
\end{aligned}
$$

The $\left(\mathrm{g}_{N}, \lambda\right)$ flow is highly non-universal but the flow of the scale invariant combination $\tau=\mathrm{g}_{N} \lambda$

$$
(4 \pi)^{2} \mu \frac{d}{d \mu} \tau=2 s \tau u(\omega)+\frac{s^{2}}{8 \omega^{2}}\left(1+20 \omega^{2}\right),
$$

is independent of the gauge fixing parameters and $\xi$. Note that in principle one is free to freeze the $\mathrm{g}_{N}$ evolution by taking $\xi=-s \gamma(\omega)$ and work with Planck units. As discussed in section 2.1 this amounts to using a potentially singular parametric representation of the $\tau$ flow and we prefer to let both $g_{N}$ and $\lambda$ evolve, e.g. with $\xi=0$.

The flow equations (2.22), (2.23) are also scheme dependent. The scheme dependence turns out to be crucial in the present context, so we discuss it in slightly more detail following [106]. We momentarily display the loop counting parameter $\hbar$ (not to be identified with Planck's constant here) and make use of the fact that changes of scheme correspond to finite redefinitions of the renormalized couplings compatible with the $\hbar$ grading. One readily sees that $\hbar$ and the dimensionless couplings should enter only in the combinations $\hbar s, \hbar \mathrm{g}_{N}, \lambda, \omega, \theta$. For simplicity we assume that $\omega$ and $\theta$ couplings are set 
to their values $\omega_{*}, \theta_{*}$ at their nontrivial fixed point. Taking $s$ as the reference coupling the the most general $O(\hbar)$ redefinitions compatible with the above grading then are

$$
\begin{aligned}
\tilde{\mathrm{g}}_{N} & =\mathrm{g}_{N}+\hbar\left(c_{1} \mathrm{~g}_{N}^{2}+c_{2} \mathrm{~g}_{N} s+c_{3} s^{2}\right), \\
\tilde{\lambda} & =\lambda+\hbar\left(d_{1} \mathrm{~g}_{N}+d_{2} s\right)
\end{aligned}
$$

where $c_{1}, c_{2}, c_{3}, d_{1}, d_{2}$ are functions (powerseries) of $s / \mathrm{g}_{N}, \lambda$ and $\omega_{*}, \theta_{*}$. The flow equations for the new couplings are readily worked out, after which one can restore $\hbar=1$. At $s=s_{*}=0$ the new flow equations simplify to

$$
\begin{aligned}
\mu \frac{d}{d \mu} \tilde{\mathrm{g}}_{N} & =2 \tilde{\mathrm{g}}_{N}+2 \tilde{\mathrm{g}}_{N}^{2} c_{1}, \\
\mu \frac{d}{d \mu} \tilde{\lambda} & =-2 \tilde{\lambda}+2 \tilde{\mathrm{g}}_{N}\left(2-\tilde{\lambda} \frac{\partial}{\partial \tilde{\lambda}}\right) d_{1} .
\end{aligned}
$$

The point at issue now is that these equations have a nontrivial fixed point for $\tilde{\mathrm{g}}_{N}$ whenever $c_{1} \neq 0$ and generically also one in $\tilde{\lambda}$ :

$$
\tilde{\mathrm{g}}_{N}^{*}=-\frac{1}{c_{1}}, \quad \tilde{\lambda}^{*}=0 \quad \text { or }\left.\quad \tilde{\lambda}^{2} \frac{\partial}{\partial \tilde{\lambda}}\left(\tilde{\lambda}^{-2} d_{1}\right)\right|_{\tilde{\lambda}=\tilde{\lambda}^{*}}=c_{1} \neq 0
$$

The rationale for identifying a non-Gaussian fixed point in perturbation theory is explained in Appendix A1.

There are several reasons why in the specific context here the flow equations (2.25) rather than (2.22) should be considered as indicative for the genuine $\left(\mathrm{g}_{N}, \lambda\right)$ flow: (i) Dimensional regularization suppresses powerlike divergencies in the UV cutoff, in particular singular contributions from the measure are not taken into account. On the other hand the proper treatment of measure terms is essential for the perturbative cure of the conformal factor instability [99]. (ii) Dimensional regularization has no nonperturbative counterpart, so the matching to a nonperturbatively defined $\left(\mathrm{g}_{N}, \lambda\right)$ flow should be done for the flow (2.25) with nontrivial $c_{1}, d_{1}$. (iii) A recent reevaluation of $\Gamma_{\text {div }}^{(1)}$ using tabulated heat kernels asymptotics [38] confirmed the presence of powerlike singularities leading to additional terms in the flow equations (2.22). These can be checked [106] to correspond to a change of scheme (2.24) with suitable $c_{1}=c_{1}(\omega), d_{1}=d_{10}(\omega)+d_{11}(\omega) \tilde{\lambda}$, $d_{2}=d_{2}(\omega), c_{2}=c_{3}=0$. (iv) Upon expanding a $\left(\mathrm{g}_{N}, \lambda\right)$ flow defined through the truncated effective average action in powers of $g_{N}$ a match to the perturbative 1-loop flow should be found. This is indeed the case for (2.25) with nontrivial $c_{1}, d_{1}$.

Let us briefly elaborate on point (iv). In the so-called Einstein-Hilbert truncation using an optimed cutoff and a limiting version of the gauge-fixing parameter, the 'beta' functions $\gamma_{g}, \beta_{\lambda}$ reduce to ratios of polynomials in $\mathrm{g}_{N}, \lambda$, see Eq. (1.26). Upon expansion to 
one finds

$$
\begin{aligned}
\mu \frac{d}{d \mu} \mathrm{g}_{N} & =2 \mathrm{~g}_{N}-\frac{1}{(4 \pi)^{2}} \mathrm{~g}_{N}^{2}+O\left(\mathrm{~g}_{N}^{2} \lambda\right), \\
\mu \frac{d}{d \mu} \lambda & =-2 \lambda+\frac{1}{(4 \pi)^{2}} \frac{\mathrm{g}_{N}}{2}(1+2 \lambda)+O\left(\mathrm{~g}_{N}^{2}\right) .
\end{aligned}
$$

This is of the form $(2.25)$ with $(4 \pi)^{2} c_{1}=-1 / 2,(4 \pi)^{2} d_{1}=1 / 8+\tilde{\lambda} / 2$, with the ensued nontrivial fixed point $(2.26)$.

The flow equations (2.8), (2.18) of course also admit the Gaussian fixed point $\mathrm{g}_{N}^{*}=0=$ $\lambda_{*}$, and one may be tempted to identify the 'realm' of perturbation theory (PT) with the 'expansion' around a Gaussian fixed point. As explained in Appendix A1, however, in a theory with several couplings the conceptual status of PT referring to a non-Gaussian fixed point is not significantly different from that referring to a Gaussian fixed point. In other words there is no reason to take the perturbative non-Gaussian fixed point (2.26) any less serious than the perturbative Gaussian one. This point is also relevant in the framework of the $2+2$ reduction, where a non-Gaussian fixed point is also identified by perturbative means.

The fact that a non-Gaussian fixed point can already be identified in PT is important for several reasons. First, it provides an important consistency check on the scenario. It is only in PT that the ultraviolet cutoff can strictly be removed; granting the usual assumption that it is asymptotic to the exact result, the putative non-Gaussian fixed point should be visible already in first order PT - and it is! Second, although the value of $\mathrm{g}_{N}^{*}$ in (2.20) is non-universal, the anomalous dimension $\eta_{N}=\gamma_{g} / \mathrm{g}_{N}-2$ is exactly -2 at the fixed point (2.20). The general argument for the dimensional reduction of the residual interactions outlined after Eq. (1.7) can thus already be based on PT alone! Together the result (2.20) suggests that the interplay between the perturbative and the nonperturbative dynamics might be similar to that of non-abelian gauge theories, where the nonperturbative dynamics is qualitatively and quantitatively important mostly in the infrared.

In summary there is good evidence for the existence of a non-Gaussian fixed point in higher derivative gravity theories. Here we limited the discussion to the one loop level, the qualitative aspects should be unaffected by loop corrections, however. Indeed, the theories (2.14) are thought to be strictly renormalizable to all loop orders [138]. This is not trivially a consequence of the $1 / p^{4}$ propagator (see [12] for counter examples) but arises through the interplay with diffeomorphism invariance. By much of the same rationale that underlies the belief that Yang-Mills theories exist nonperturbatively one is then lead to the following conjecture (which, although not verbatim contained in [152] is much along the same lines):

Higher derivative gravity theories are renormalizable in $d=4$ in the Kadanoff-Wilson sense with finitely many asymptotically safe couplings based on a non-Gaussian fixed point. 


\subsection{Can unitarity and renormalizability be reconciled?}

The main drawback of the above renormalizable gravity theories is that the status of unitarity in them is uncertain. In a perturbative formulation based on the free propagator obtained by linearizing (2.14) one encounters unphysical propagating modes, see section 1.5 for a discussion of this issue. As already mentioned these problem are absent in an alternative perturbative formulation where a $1 / p^{2}$ type propagator is used throughout [66]. We now describe this construction in slightly more detail. See also [90] for a recent alternative setting.

Starting from the $d=4$ Lagrangian $\frac{1}{c_{d} G_{N}} \sqrt{q} R(q)$ without cosmological constant the one-loop divergencies come out in dimensional regularization as [140]

$$
\frac{\mu^{d-4}}{(4 \pi)^{2}(d-4)} \sqrt{q}\left(\frac{1}{60} R^{2}+\frac{7}{10} R_{\alpha \beta} R^{\alpha \beta}\right) .
$$

They can be removed in two different ways. One is by adding new couplings so that a higher derivative action of the form (2.14) arises with parameters $\Lambda=0, x=0$, and

$$
z_{B}=\mu^{d-4}\left(z+\frac{1}{(4 \pi)^{2}(d-4)} \frac{1}{60}\right), \quad y_{B}=\mu^{d-4}\left(y+\frac{1}{(4 \pi)^{2}(d-4)} \frac{7}{10}\right) .
$$

The renormalizability of the resulting theory is mostly due to the modified propagator which can be viewed as a resummed graviton propagator in a power series in $z, y$. The unphysical singularities are of order $1 / z, 1 / y$. The second option to remove (2.28) is by a singular field redefinition

$$
q_{\alpha \beta} \mapsto q_{\alpha \beta}+\frac{c_{4} G_{N}}{(4 \pi)^{2}(d-4)} \frac{1}{10}\left(-7 R_{\alpha \beta}+\frac{11}{3} g_{\alpha \beta} R\right) .
$$

This restores the original $\sqrt{q} R(q)$ Lagrangian up to two- and higher loop contributions. However this feature is specific to one loop. As shown in $[67,157]$ at two loops there is a divergence proportional to $R_{\alpha \beta}^{\gamma \delta} R_{\rho \sigma}^{\alpha \beta} R_{\gamma \delta}^{\rho \sigma}$, which cannot be absorbed by a field redefinition. A counterterm proportional to it must thus be added to $\sqrt{q} R(q)$. Importantly, when re-expanded in powers of $f_{\alpha \beta}=q_{\alpha \beta}-\delta_{\alpha \beta}$, this counterterm, however, produces only terms quadratic in $f$ that are proportional to the Ricci tensor or the Ricci scalar. These can be removed by a covariant field redefinition, so that the initial $1 / p^{2}$ type propagator does not receive corrections. A simple argument [8] shows that this property also holds for all higher order counterterms that can be expected to occur. Explicitly, consider a Lagrangian of the form

$$
L=\frac{1}{c_{d} G_{N}} \sqrt{q} R(q)+\sum_{i \geq 1}\left(c_{d} G_{N}\right)^{\frac{d_{i}}{2-d}-d} \mathrm{~g}_{i} L_{i}(q)
$$


where $L_{i}(q)$ are local curvature invariants of mass dimension $-d_{i}$, the $g_{i}$ are dimensionless couplings and the power of $c_{d} G_{N}$ (with $c_{d}$ e.g. as in (2.4)) gives each term in the sum mass dimension $-d$.

Let us briefly recap the power counting and scaling dimensions of local curvature invariants. These are integrals $P_{i}[g]=\int d^{d} x L_{i}(g)$ over densities $L_{i}(g)$ which are products of factors of the form $\nabla_{\alpha_{1}} \ldots \nabla_{\alpha_{l-4}} R_{\alpha_{l-3} \ldots \alpha_{l}}$, suitably contracted to get a scalar and then multiplied by $\sqrt{g}$. One easily checks $L_{i}\left(\omega^{2} g\right)=\omega^{s_{i}} L_{i}(g), \omega>0$, with $s_{i}=d-2 r-s$, where $r$ is the total power of the Riemann tensor and $s$ is the (necessarily even) total number of covariant derivatives. This scaling dimension matches minus the mass dimension of $P_{i}(g)$ if $g$ is taken dimensionless. For the mass dimension $d_{i}$ of the associated coupling $u_{i}$ in a product $u_{i} P_{i}[g]$ one thus gets $d_{i}=s_{i}=d-2 r-s$. For example, the three local invariants in (1.24) have mass dimensions $-d_{0}=-d,-d_{1}=-(d-2)$, $-d_{2}=-(d-4)$, respectively. There are three other local invariants with mass dimension $-(d-4)$, namely the ones with integrands $C^{2}=R^{\alpha \beta \gamma \delta} R_{\alpha \beta \gamma \delta}-2 R^{\alpha \beta} R_{\alpha \beta}+R^{2} / 3$ (the square of the Weyl tensor), $E=R^{\alpha \beta \gamma \delta} R_{\alpha \beta \gamma \delta}-4 R^{\alpha \beta} R_{\alpha \beta}+R^{2}$ (the generalized Euler density), and $\nabla^{2} R$. Then there is a set of dimension $-(d-6)$ local invariants, and so on. Note that in $d=4$ the integrands of the last two of the dimensionless invariants are total divergencies so that in $d=4$ there are only 4 local invariants with non-positive mass dimension; see (2.14).

A generic term in $P_{i}$ will be symbolically of the form $\nabla^{s} R^{r}$, where all possible contractions of the $4 r+s$ indices may occur. Since the Ricci tensor is schematically of the form $R=\nabla^{2} f+O\left(f^{2}\right)$, the piece in $P_{i}$ quadratic in $f$ is of the form $\nabla^{s+4} R^{r-2} f^{2}$. The coefficient of $f^{2}$ is a tensor with 4 free indices and one can verify by inspection that the possible index contractions are such that the Ricci tensor or Ricci scalar either occurs directly, or after using the contracted Bianchi identity. In summary, one may restrict the sum in (2.24) to terms with $-d_{i}=-d+2 r+s, r \geq 3$, and the propagator derived from it will remain of the $1 / p^{2}$ type to all loop orders. This suggests that $(2.24)$ will give rise to a renormalizable Lagrangian. A proof requires to show that after gauge fixing and ghost terms have been included all counter terms can be chosen local and covariant and has been given in [66].

In the terminology of section 1.4 the above results then show the existence of a "weakly renormalizable" but "propagator unitary" Quantum Gravidynamics based on a perturbative Gaussian fixed point. The beta functions for this infinite set of couplings are presently unknown. If they were known, expectations are that at least a subset of the couplings would blow up at some finite momentum scale $\mu=\mu_{\text {term }}$ and would be unphysical for $\mu>\mu_{\text {term }}$. In this case the computed results for physical quantities are likely to blow up likewise at some (high) energy scale $\mu=\mu_{\text {term }}$. In other words the couplings in (2.24) are presumably not all asymptotically safe.

Let us add a brief comment on the relevant-irrelevant distinction in this context, if only to point out that it is no longer useful. Recall from Section 1.3 that the notion of a relevant or irrelevant coupling applies even to flow lines not connected to a fixed 
point. This is the situation here. All but a few of the interaction monomials in (2.24) are powercounting irrelevant with respect to the $1 / p^{2}$ propagator. Equivalently all but a few couplings $u_{i}(\mu)=\mu^{d_{i}} \mathrm{~g}_{i}(\mu)$ have non-negative mass dimensions $d_{i} \geq 0$. These are the only ones not irrelevant with respect to the stability matrix computed at the perturbative Gaussian fixed point. However in (2.24) these power counting irrelevant couplings with $d_{i}<0$ are crucial for the absorption of infinities and thus are converted into practically relevant ones. In the context of (2.24) we shall therefore discontinue to use the terms relevant/irrelevant.

Comparing the two perturbative constructions in section 2.2 and 2.3 one arrives at the conclusion anticipated in section 1.1: the challenge of Quantum Gravidynamics lies not so much in achieving renormalizability, but to reconcile asymptotically safe couplings with the absence of unphysical propagating modes. As surveyed in section $1.3 \mathrm{c}$ this program is realized for the $2+2$ reduction $[108,109]$. Within the framework of truncated flow equations (see section $1.3 \mathrm{~d}$ and $\mathrm{A} 2$ in section 1.5 here) the results for the $R+R^{2}$ type truncation likewise are compatible with the absence of unphysical propagating modes.

\section{Dimensional reduction of residual interactions in UV}

In order to realize this program without reductions or truncations a mathematically controllable nonperturbative definition of Quantum Gravidynamics is needed. Within a functional integral formulation this involves the following main steps: definition of a kinematical measure, setting up a coarse graining flow for the dynamical measures and then probing its asymptotic safety. This is probably best done in a discretized setting. However an important qualitative feature of an asymptotically safe functional integral can be inferred without actually evaluating it, namely that in the extreme ultraviolet the residual interactions appear two-dimensional. There are a number of interconnected heuristic arguments for this phenomenon which we present here.

(a) Scaling of fixed point action: Consider a candidate for a quasilocal microscopic action

$$
S_{k}[q]=\sum_{i} u_{i}(k) P_{i}[q]
$$

where the $u_{i}(k)$ are running couplings of mass dimension $d_{i}$ and $P_{i}[q]$ are local invariants of mass dimension $-d_{i}$. By quasilocal we mean here that the sum may be infinite and off hand arbitrarily high derivative terms may occur. For example such an action arises in the perturbative framework described in section 2.3. When viewed as a renormalized action perturbatively defined in the above sense (with the UV cutoff strictly removed) the running of the $u_{i}^{\mathrm{PT}}(k)$ is unknown but expectations are that $\mathrm{g}_{i}^{\mathrm{PT}}(k)=k^{-d_{i}} u_{i}^{\mathrm{PT}}(k)$ are not uniformly bounded functions in $k$, the dimensionless couplings then are not 
asymptotically safe but blow up at various ( $i$-dependent) intermediate scales. The situation is drastically different if all the couplings are assumed to be asymptotically safe. Then $u_{i}(k)=\mathrm{g}_{i}(k) k^{d_{i}} \sim \mathrm{g}_{i}^{*} k^{d_{i}}$ as $k \rightarrow \infty$ and if one uses the fact that $s_{i}=d_{i}$ (see the discussion after Eq. (2.24) for all local invariants one gets

$$
S_{k}[q] \sim \sum_{i} \mathrm{~g}_{i}^{*} P_{i}\left[k^{2} q\right]=S_{*}\left[k^{2} q\right]
$$

for $k \rightarrow \infty$, with $S_{*}[q]=\sum_{i} \mathrm{~g}_{i}^{*} S_{i}[q]$ the candidate fixed point action. The overall scale of the metric is an inessential parameter, see section 2.1, and a fixed point action always refers to an equivalence class modulo possibly running inessential parameters.

One sees that in the fixed point regime $\mathrm{g}_{i}(k) \sim \mathrm{g}_{i}^{*}$ the $k$-dependence enters only through the combination $k^{2} g_{\alpha \beta}$, a kind of selfsimilarity. This simple but momentous fact eventually underlies all the subsequent arguments. It is 'as if' in the fixed point regime only a rescaled metric $\tilde{q}_{\alpha \beta}=k^{2} q_{\alpha \beta}$ entered which carries dimension two. This has consequences for the 'effective dimensionality' of Newton's constant: recall that conventionally the Ricci scalar term, $\int d x \sqrt{q} R(q)$, has dimension $2-d$ in $d$ dimensions. Upon substitution $q_{\alpha \beta} \mapsto \tilde{q}_{\alpha \beta}$ one quickly verifies that $\int d x \sqrt{\tilde{q}} R(\tilde{q})$ is dimensionless. Its prefactor, i.e. the inverse of Newton's constant, then can be taken dimensionless - as it is in two dimensions. Compared to the infrared regime it looks 'as if' Newton's constant changed its effective dimensionality from $d-2$ to zero, i.e. at the fixed point there must be a large anomalous dimension $\eta_{N}=2-d$.

Formally what is special about the Einstein-Hilbert term is that the kinetic (second derivative) term itself carries a dimensionful coupling. To avoid the above conclusion one might try to assign the metric a mass dimension 2 from the beginning (i.e. not just in the asymptotic regime). However this would merely shift the effect from the gravity to the matter sector, as we wish to argue now.

In addition to the dimensionful metric $\tilde{q}_{\alpha \beta}:=k^{2} q_{\alpha \beta}$, we introduce a dimensionful vielbein by $\tilde{E}_{\alpha}^{m}:=k E_{\alpha}^{m}$, if $q_{\alpha \beta}=E_{\alpha}{ }^{m} E_{\beta}{ }^{n} \eta_{m n}$ is the dimensionless metric. With respect to a dimensionless metric $\int d x \sqrt{q} R(q)$ has mass dimension $2-d$ in $d$ dimensions, while the mass dimensions $d_{\chi}$ of a Bose field $\chi$ and that $d_{\psi}$ of a Fermi field $\psi$ are set such that their kinetic terms are dimensionless, i.e. $d_{\chi}=(d-2) / 2$ and $d_{\psi}=(d-1) / 2$. Upon substitution $q_{\alpha \beta} \mapsto \tilde{q}_{\alpha \beta}$ the gravity part $\int d x \sqrt{\tilde{q}} R(\tilde{q})$ becomes dimensionless, while the kinetic terms of a Bose and Fermi field pick up a mass dimension of $d-2$ and $d-1$, respectively. This means their wave function renormalization constants $Z_{\chi}(k)$ and $Z_{\psi}(k)$ are now dimensionful and should be written in terms of dimensionless parameters as $Z_{\chi}(k)=k^{d-2} / \mathrm{g}_{\chi}(k)$ and $Z_{\psi}(k)=k^{d-1} / \mathrm{g}_{\psi}(k)$, say. For the dimensionless parameters one expects finite limit values $\lim _{k \rightarrow \infty} \mathrm{g}_{\chi}(k)=\mathrm{g}_{\chi}^{*}>0$ and $\lim _{k \rightarrow \infty} \mathrm{g}_{\psi}(k)=\mathrm{g}_{\psi}^{*}>0$, since otherwise the corresponding (free) field would simply decouple. Defining the anomalous dimension as usual $\eta_{\chi}=-k \partial_{k} \ln Z_{\chi}$ and $\eta_{\psi}=-k \partial_{k} \ln Z_{\psi}$, the argument presented after Equation (1.7) can be repeated and gives that $\eta_{\chi}^{*}=2-d, \eta_{\psi}^{*}=1-d$ for the fixed point values, respectively. The original large momentum behavior $1 / p^{2}$ for bosons and $1 / p$ for 
fermions is thus modified to a $1 / p^{d}$ behavior in the fixed point regime, in both cases.

This translates into a logarithmic short distance behavior which is universal for all (free) matter. Initially the propagators used here should be viewed as "test propagators", in the sense that one transplants the information in the $\eta$ 's derived from the gravitational functional integral into a conventional propagator on a (flat or curved) background spacetime. Since the short distance asymptotics is the same on any (flat or curved) reference spacetime, one can plausibly convert this into a prediction for a genuine quantum gravity correlator:

Consider as in [40] a geodesic two-point correlator of a scalar field

$$
G(R)=\int \mathcal{D} q \mathcal{D} \phi e^{i S[q, \phi]} \int d x d y \sqrt{q(x)} \sqrt{q(y)} \phi(x) \phi(y) \delta\left(\Sigma_{q}(x, y)-R\right),
$$

where $\Sigma_{q}(x, y)$ is the minimal geodesic distance between the points $x$ and $y$. The first integral is the heuristic geometry and matter functional integral, all configurations are taken into account which produce the given geodesic distance $R$. The previous considerations then lead to the prediction that if (3.3) is based on an asymptotically safe functional measure a logarithmic (powers of $\log R$ and $\log (-\log R)$ ) behavior for $R \rightarrow 0$ is expected.

On the other hand the universality of the logarithmic short distance behavior in the matter propagators also justifies to attribute the phenomenon to a modification in the underlying random geometry, a kind of "quantum equivalence principle".

(b) Anomalous dimension at non-Gaussian fixed point: The "anomalous dimension argument" has already been sketched in the introduction, see also [94]. Here we present a few more details and relate it to (a).

Suppose again that the unkown microscopic action of Quantum Gravidynamics is quasilocal and reparameterization invariant. The only term containing second derivatives then is the familiar Einstein-Hilbert term, $Z_{N} \int d x \sqrt{q} R(q)$, of mass dimension 2- $d$ in $d$ dimensions, if the metric is taken dimensionless. As explained in section 2.1 the dimensionful running prefactor multiplying it, $Z_{N}(k),(N$ for "Newton") can be treated either as a wave function renormalization or as a quasi-essential dimensionless coupling $\mathrm{g}_{N}$, where

$$
c_{d} G_{\mathrm{N}}=Z_{N}(k)^{-1}=\mathrm{g}_{N}(k) k^{2-d} .
$$

Here we treat $\mathrm{g}_{N}$ as running in which case its running may also be affected by all the other couplings (gravitational and non-gravitational, made dimensionless by taking out a suitable power of $k$ ). The short distance behavior of the propagator will now be governed by the "anomalous dimension" $\eta_{N}=-k \partial_{k} \ln Z_{N}(k)$, by general field theoretical arguments. On the other hand the flow equation for $\mathrm{g}_{N}$ can be expressed in terms of $\eta_{N}$ as $k \partial_{k} \mathrm{~g}_{N}=\left[d-2+\eta\left(\mathrm{g}_{N}\right.\right.$, other $\left.)\right] \mathrm{g}_{N}$, where we schematically indicated the dependence 
on the other dimensionless couplings. If this flow equation now has a nontrivial fixed point $\infty>\mathrm{g}_{N}^{*}>0$, the only other way how the right hand side can vanish is for

$$
\eta_{N}\left(\mathrm{~g}_{N}^{*}, \text { other }\right)=2-d
$$

irrespective of the detailed behavior of the other couplings as long as no blow-up occurs. This is a huge anomalous dimension. We can now transplant this anomalous dimension into a "test graviton propagator" on a flat background. The characteristic property of $\eta_{N}$ then is that it gives rise to a a high momentum behavior of the form $\left(p^{2}\right)^{-1+\eta_{N} / 2}$ modulo logarithms, or a short distance behavior of the form $\left(\sqrt{x^{2}}\right)^{2-d-\eta_{N}}$ modulo logarithms. This follows from general field theoretical principles: a Callan-Symanzik equation for the effective action, the vanishing of the beta function at the fixed point and the decoupling of the low momentum modes. Keeping only the leading part the vanishing power at $\eta_{N}=2-d$ translates into a logarithmic behavior, $\ln x^{2}$, formally the same as for a massless scalar propagator in a two-dimensional field theory.

The fact that a large anomalous dimension occurs at a non-Gaussian fixed point was initially observed in the context of the $2+\epsilon$ expansion $[83,84]$ and later in computations based on the effective average action [94, 95]. The above argument shows that no specific computational information enters.

Let us emphasize that in general an anomalous dimension is not related to the geometry of field propagation and in a conventional field theory one cannot sensibly define a fractal dimension by looking at the high momentum behavior of a two-point function [91]. What is special about gravity is ultimately that the propagating field itself defines distances. One aspect thereof is the universal way matter is affected, as seen in (a). In contrast to an anomalous dimension in conventional field theories, this allows one to attribute a geometric significance to the modified short distance behavior of the test propagators, see $(d)$.

(c) Strict renormalizability and $1 / p^{4}$ propagators: With hindsight the above patterns are already implicit in earlier work on strictly renormalizable gravity theories. As emphasized repeatedly the benign renormalizability properties of higher derivative theories are mostly due to the use of $1 / p^{4}$ type propagator (in $d=4$ dimensions). As seen in section 2.2 this $1 / p^{4}$ type behavior goes hand in hand with asymptotically safe couplings. Specifically for the dimensionless Newton's constant $\mathrm{g}_{N}$ it is compatible with the existence of a nontrivial fixed point, see (2.20). This in turn enforces an anomalous dimension $\eta_{N}=-2$ at the fixed point which links back to the $1 / p^{4}$ type propagator.

Similarly in the $1 / N$ expansion $[145,146,135]$ a nontrivial fixed point goes hand in hand with a propagator whose high momentum behavior is of the form $1 /\left(p^{4} \ln p^{2}\right)$, in four dimensions, and formally $1 / p^{d}$ in $d$ dimensions. In position space this amounts to a $\ln x^{2}$ behavior, once again.

(d) Spectral dimension and scaling of fixed point action: The scaling (3.2) of the fixed point action also allows one to estimate the behavior of the spectral dimension 
in the ultraviolet. This leads to a model-independent variant [106] of an argument used in $[96,97]$.

If one wants to probe the functional measure over geometries only an interesting operator insertion is the trace of the heat kernel $[82,85,5]$

$$
G(T)=\int \mathcal{D} q e^{i S[q]} \int d x \sqrt{q(x)} \exp \left(T \Delta_{q}\right)(x, x)
$$

Here $\Delta_{q}:=q^{\alpha \beta} \nabla_{\alpha} \nabla_{\beta}=\sqrt{q}^{-1} \partial_{\alpha}\left(\sqrt{q} q^{\alpha \beta} \partial_{\beta}\right)$ is the Laplace-Beltrami operator, and the heat kernel $\exp \left(T \Delta_{q}\right)\left(x, x^{\prime}\right)$ associated with it is the symmetric (in $x, x^{\prime}$ ) bi-solution of the heat equation $\partial_{T} K=\Delta_{q} K$ with initial condition $\lim _{T \rightarrow 0} \exp \left(T \Delta_{q}\right)\left(x, x^{\prime}\right)=\delta\left(x, x^{\prime}\right)$. The $T \rightarrow \infty$ limit will then probe the large scale structure of the typical geometries in the measure and the $T \rightarrow 0$ limit will probe the micro aspects. Again the expressions (3.6) are here only heuristic, in particular normalization factors have been omitted and the functional measure over geometries would have to be defined as previously outlined.

Let us briefly recapitulate the definition of the heat kernel and some basic properties. For a smooth Riemannian metric $g$ on a compact closed $d$-manifold let $\Delta_{g}:=g^{\alpha \beta} \nabla_{\alpha} \nabla_{\beta}=$ $\sqrt{g}^{-1} \partial_{\alpha}\left(\sqrt{g} g^{\alpha \beta} \partial_{\beta}\right)$ be the Laplace-Beltrami operator. The heat kernel $\exp \left(T \Delta_{g}\right)\left(x, x^{\prime}\right)$ associated with it is the symmetric (in $x, x^{\prime}$ ) bi-solution of the heat equation $\partial_{T} K=\Delta_{g} K$ with initial condition $\lim _{T \rightarrow 0} \exp \left(T \Delta_{g}\right)\left(x, x^{\prime}\right)=\delta\left(x, x^{\prime}\right)$. Since $(\mathcal{M}, g)$ is compact $\Delta_{g}$ has purely discrete spectrum with finite multiplicities. We write $-\Delta_{g} \phi_{n}(g)=\mathcal{E}_{n}(g) \phi_{n}(g)$, $n \geq 0$, for the spectral problem and assume that the eigenfunctions $\phi_{n}$ are normalized and the eigenvalues monotonically ordered $\mathcal{E}_{n}(g) \leq \mathcal{E}_{n+1}(g)$. We write $V(g)=\int d x \sqrt{g}$ for the volume of $(\mathcal{M}, g)$ and

$$
P_{g}(T)=\frac{1}{V(g)} \int d x \sqrt{g} \exp \left(T \Delta_{g}\right)(x, x)=\frac{1}{V(g)} \sum_{n} e^{-\mathcal{E}_{n}(g) T}
$$

for the trace of the heat kernel. In the random walk picture $P_{g}(T)$ can be interpreted as the probability of a test particle diffusing away from a point $x \in \mathcal{M}$ and to return to it after the fictitious diffusion time $T$ has elapsed. In flat Euclidean space $(\mathcal{M}, g)=\left(\mathbb{R}^{d}, \eta\right)$ for example $P_{\eta}(T)=(4 \pi T)^{-d / 2}$ for all $T$. For a generic manifold the trace of the heat kernel cannot be evaluated exactly. However the short time and the long time asymptotics can to some extent be described in closed form. Clearly the $T \rightarrow \infty$ limit probes the large scale structure of a Riemannian manifold (small eigenvalues $\mathcal{E}_{n}(g)$ ) while the $T \rightarrow 0$ limit probes the small scales (large eigenvalues $\mathcal{E}_{n}(g)$ ).

For $T \rightarrow 0$ one has an asymptotic expansion $P_{g}(T) \sim(4 \pi T)^{-d / 2} \sum_{n \geq 0} T^{n} \int d x \sqrt{g} a_{n}(x)$, where the $a_{n}$ are the Seeley-deWitt coefficients. These are local curvature invariants, $a_{0}=1, a_{1}=\frac{1}{6} R(g)$, etc. The series can be rearranged so as to collect terms with a fixed power in the curvature or with a fixed number of derivatives [14]. Both produces nonlocal curvature invariants. The second rearrangement is relevant when the 
curvatures are small but rapidly varying (so that the derivatives of the curvatures are more important then their powers). The leading derivative terms then are given by $P_{g}(T) \sim(4 \pi T)^{-d / 2}\left[V(g)+T \int d x \sqrt{g} a_{1}+T^{2} N_{2}(T)+\ldots\right]$, where $N_{2}(T)$ is a known nonlocal quadratic expression in the curvature tensors. The $T \rightarrow \infty$ behavior is more subtle as also global information on the manifold enters. For compact manifolds a typical behavior is $P_{g}(T) \sim(4 \pi T)^{-d / 2}[1+O(\exp (-c T))]$, where the rate of decay $c$ of the subleading term is governed by the smallest non-zero eigenvalue.

Returning now to the quantum gravity average $G(T) \sim\left\langle P_{q}(T)\right\rangle$, one sees that on any state on which all local curvature polynomials vanish the leading short distance behavior of $\left\langle P_{q}(T)\right\rangle$ will always be $\sim T^{-d / 2}$, as on a fixed manifold. The same will hold if the nonlocal invariants occurring in the derivative expansion all have vanishing averages in the state considered. A leading short distance behavior of the form

$$
\left\langle P_{q}(T)\right\rangle \sim T^{-d_{s} / 2}, \quad T \rightarrow 0
$$

with $d_{s} \neq d$ will thus indicate that either the operations "taking the average" and "performing the asymptotic expansion for $T \rightarrow 0$ " no longer commute, or that the microscopic geometry is very rough so that the termwise averages no longer vanish, or both. Whenever well-defined the quantity $d_{s}(T):=-2 d \ln \left\langle P_{q}(T)\right\rangle / d \ln T$ is known as the spectral dimension (of the micro-aspects of the random geometries probed by the state $\mathcal{O} \mapsto\langle\mathcal{O}\rangle)$. See $[82,85,5]$ for earlier uses in random geometry, and [19] for an evaluation of the spectral dimension for diffusion on the Sierpinski gasket based on a principle similar to (3.11) below.

We assume now that the states considered are such that the $T \rightarrow \infty$ behavior of $\left\langle P_{g}(T)\right\rangle$ is like that in flat space, i.e. $\left\langle P_{q}(T)\right\rangle \sim T^{-d / 2}$ for $T \rightarrow \infty$, see Eq. (1.11). Since $(4 \pi T)^{-d / 2}=\int \frac{d^{d} p}{(2 \pi)^{d}} \exp \left(-p^{2} T\right)$ one can give the stipulated $T \rightarrow \infty$ asymptotics an interpretation in terms of the spectrum $\left\{p^{2}, p \in \mathbb{R}^{d}\right\}$ of the Laplacian of a 'typical' reference metric $\bar{g}_{\alpha \beta}$ which is smooth and almost flat at large scales. The spectrum of $\Delta_{q}$ must be such that the small spectral values can be well approximated by $\left\{p^{2}<\right.$ $\left.C, p \in \mathbb{R}^{d}\right\}$ for some constant $C>0$. Its unknown large eigenvalues will then determine the short distance behavior of $\left\langle P_{q}(T)\right\rangle$. We can incorporate this modification of the spectrum by introducing a function $F_{\bar{g}}\left(p^{2}\right)$ which tends to 1 for $p^{2} \rightarrow 0$, and whose large $p^{2}$ behavior remains to be determined. Thus

$$
\left\langle P_{q}(T)\right\rangle \approx \int \frac{d^{d} p}{(2 \pi)^{d}} \exp \left\{-p^{2} F_{\bar{g}}\left(p^{2}\right) T\right\}
$$

The following argument now suggests that within the asymptotic safety scenario $F_{\bar{g}}\left(p^{2}\right) \sim$ $p^{2}$ for $p^{2} \rightarrow \infty$. Before turning to the argument let us note that this property of $F_{\bar{g}}\left(p^{2}\right)$ entails

$$
\left\langle P_{q}(T)\right\rangle \sim T^{-d / 4} \quad \text { for } \quad T \rightarrow 0, \quad \text { i.e. } d_{s}=d / 2 \text {. }
$$


The "microscopic" spectral dimension equals half the "macroscopic" $d$. Notably this equals 2, as suggested by the "anomalous dimension argument" precisely in $d=4$ dimensions.

The argument for $F_{\bar{g}}\left(p^{2}\right) \sim p^{2}$ for $p^{2} \rightarrow \infty$ goes as follows: We return to discrete description $P_{q}(T)=\sum_{n} e^{-\mathcal{E}_{n}(q) T}$ for $(\mathcal{M}, q)$ compact, and consider the average of one term in the sum $\left\langle e^{-\mathcal{E}_{n}(q) T}\right\rangle$, with $\mathcal{E}_{n}(q)$ large. The computation of this average is a single scale problem in the terminology of Appendix A. As such it should allow for a good description via an effective field theory at scale $k$. Here only the fact is needed that the average $\left\langle e^{-\mathcal{E}_{n}(q) T}\right\rangle$ can approximately be evaluated as $[96,97]$

$$
\left\langle e^{-\mathcal{E}_{n}(q) T}\right\rangle \approx e^{-\mathcal{E}_{n}\left(\check{g}_{k}\right) T}
$$

where $\left(\check{g}_{k}\right)_{\alpha \beta}$ is a saddle point configuration of the effective field theory at scale $k$, defined e.g. as a stationary point of the effective action $\bar{\Gamma}_{k}[g]$ at scale $k$, see the discussion at the end of Section 1.2 Since the only scale available is $\mathcal{E}_{n}$ itself the relevant scale $k$ is for given $n$ determined by the implicit equation $k^{2}=\mathcal{E}_{n}\left(\check{g}_{k}\right)$. Next we consider how these spectral values scale in the fixed point regime where the dimensionless couplings are approximately constant, $\mathrm{g}_{i}(k) \approx \mathrm{g}_{i}$. Recall from $(3.2)$ the limiting behavior $\bar{\Gamma}_{k}[g] \rightarrow$ $S_{*}\left[k^{2} g\right]$ as $k \rightarrow \infty$. Two stationary points $\left(\check{g}_{k}\right)_{\alpha \beta}$ for $\bar{\Gamma}_{k}$ and $\left(\check{g}_{k_{0}}\right)_{\alpha \beta}$ for $\bar{\Gamma}_{k_{0}}$ will thus in the fixed point regime be simply related by $k^{2} \check{g}_{k}=k_{0}^{2} \check{g}_{k_{0}}$. Since $k^{2} \Delta_{k^{2} g}=\Delta_{g}$ this means for the spectral values $k^{2} \mathcal{E}_{n}\left(\check{g}_{k}\right)=k_{0}^{2} \mathcal{E}_{n}\left(\check{g}_{k_{0}}\right)$. In order to make contact to the continuum parameterization in (3.9) we now identify for given $p$ the $n$ 's such that for the typical metric $\bar{g}_{\alpha \beta}$ entering (3.9) one has $\mathcal{E}_{n}(\bar{g}) \sim p^{2}$ for large $n$. After this reparameterization $\mathcal{E}_{n}=\mathcal{E}_{p}, p=\sqrt{p^{2}}$, one can identify the $F_{\bar{g}}\left(p^{2}\right)$ in $(3.9)$ with $F_{\bar{g}}\left(p^{2}\right)=\mathcal{E}_{p}\left(\check{g}_{k=p}\right) / \mathcal{E}_{p}\left(\check{g}_{k_{0}}\right)$. This scales for $p \rightarrow \infty$ like $p^{2}$, which completes the argument.

In summary, the asymptotic safety scenario leads to the specific (theoretical) prediction that the residual interactions in the exteme ultraviolet are effectively two-dimensional. One manifestation is that the (normally powerlike) shortdistance singularities of all free matter propagators are softened to logarithmic ones. In quantum gravity averages like $G(R)$ in (3.3) this leads to the expectation that they should scale like logarithmically (powers of $\log R$ and $\log (-\log R)$ ) for $R \rightarrow 0$. On the other hand this universality allows one to shuffle the effect from matter to gravity propagators. This justifies to attribute the effect to a modification in the underlying random geometry. The spectral dimension $G(T)$ of the random geometries probed by a certain class of "macroscopic" states comes out $d / 2$, which (notably!) equals 2 precisely in $d=4$ dimensions. Technically all aspects (a) - (d) have their origin in the scaling relation (3.2).

Accepting this dimensional reduction in the extreme ultraviolet as a working hypothesis one is lead to the following conjecture: The functional averages of an asymptotically safe theory of quantum gravity can in the extreme UV be approximately (but more and more accurately as one approaches the fixed point) reproduced by a two-dimensional statistical field theory with the following properties: (i) It is two-dimensional and selfinteracting; the latter because of the non-Gaussian nature of the original fixed point. 
(ii) It is not a conformal field theory in the usual sense, as the extreme UV regime in the original theory is reached from outside the critical surface ("massive continuum limit"). (iii) It is asymptotically safe itself (accounting for the antiscreening behavior presumed to be responsible for the stabilization of the UV properties).

Note that in principle the identification of such a UV field theory is a well-posed problem. Presupposing that the functional integral has been made well-defined and through suitable operator insertions data for its extreme UV properties have been obtained, for any proposed field theory with the properties (i) -(iii) one can test whether or not these data are reproduced.

\section{Conclusions}

The asymptotic safety scenario delineates conditions under which a functional integral based quantum theory of gravity can be viable beyond the level of an effective field theory. It combines the lessons drawn from an advanced quantum field theory perspective on the problem of quantum gravity in an apparently selfconsistent way. The moral drawn from renormalization is that the main challenge lies not so much in achieving renormalizability but to reconcile asymptotically safe couplings with the absence of unphysical propagating modes. The asymptotic safety property should lead to a dynamical reduction of the interacting degrees of freedom in the extreme ultraviolet. Finally this exteme UV regime is conjectured to be described by an effectively two-dimensional quantum field theory.

The goal of this review would be reached if a reasonably convincing case for this 'heretically orthodox' scenario has been made. Future work will have to focus on four areas: (i) Consolidating the existence of a non-Gaussian fixed point and the asymptotic safety property of the couplings. This may be done in various formalisms, field variables, and approximations. (ii) Clarifying the microstructure of the geometries and identification of the antiscreening degrees of freedom. (iii) Understanding of the physically adequate notion of unitarity and its interplay with (i) and (ii). (iv) Characterization of generic observables and working out sound consequences for the macrophysics.

Acknowledgements: I wish to thank M. Reuter for the collaboration on the Living Review article with the same title. I am grateful to E. Seiler for a critical reading of the manuscript and for suggesting various improvements. In addition I wish to thank A. Ashtekar, C. Bervillier, R. Loll, E. Mottola, and A. Niemi for comments and correspondence. 


\section{A: Renormalizing the nonrenormalizable}

The modern view of renormalization has been shaped by Kadanoff and Wilson. See [81] and $[154,155,156]$ for first hand accounts and a guide to the original articles. In the present context the relevance of a Kadanoff-Wilson view on renormalization is two-fold: first it allows one to formulate the notion of renormalizability without reference to perturbation theory, and second it allows one to treat at least in principle renormalizable and nonrenormalizable theories on the same footing. For convenience we briefly summarize the main principles of the Kadanoff-Wilson approach to renormalization here:

\section{Kadanoff-Wilson view on renormalization - main principles:}

(i) A theory is not defined in terms of a given action, but in terms of a field content and the steps (ii)-(v) below.

(ii) The functional integral is performed in piecemeal, integrating out fast modes, retaining slow modes, while keeping the values of observables fixed. This "coarse graining" process results in a flow in the space of actions which depends on the chosen coarse graining operation.

(iii) Starting from a retroactively justified initial action ideally all interaction monomials generated by the flow are included in a typical action; in any case many more than just the power-counting renormalizable ones. Then one classifies the coefficients of the monomials into essential (couplings) and inessential (field redefinitions).

(iv) A fixed point (FP) in the flow of couplings is searched for. The position of the FP depends on the chosen coarse graining operation, but the rates of approach to it typically do not ("universality").

(v) The flow itself decides which monomials are relevant in the vicinity of a FP and hence defines the dynamics. The scaling dimensions with respect to a non-Gaussian FP may be different from (corrected) power-counting dimensions referring to the Gaussian FP.

(vi) The dimension of the unstable manifold and hence the "degree" of renormalizability depends on the FP!

We add some remarks: The more familar perturbative notion of renormalizability is neither sufficient (e.g. $\Phi_{4}$ theory in $d=4$ ) nor necessary (e.g. Gross-Neveu model in $d=3$ ) for renormalizability in the above sense. The title of this Section is borrowed from a paper by Gawedzki and Kupiainen [61].

As summarized here, these principles describe the construction of a so-called massive continuum limit of a statistical field theory initially formulated on a lattice, say. A brief 
reminder: in a lattice field theory there is typically a dynamically generated scale, the correlation length $\xi$, which allows one to convert lattice distances into a physical length scale, such that say, $\xi$ lattice spacings equal $1 \mathrm{fm}$. The lattice points $n=\left(n_{1}, \ldots, n_{d}\right) \in$ $\mathbb{Z}^{d}$ are then traded for dimensionful distances $x_{i}=\left(n_{i} / \xi\right)$ fm. Taking the lattice spacing to zero amounts to sending $\xi$ to infinity while keeping $x_{i}$ fixed. If the correlation functions of some lattice fields are rescaled accordingly (including a 'wave function' renormalization factor) and the limit exists this defines a massive continuum limit of the lattice theory.

The rationale for the piecemeal performance of the functional integral is that in statistical mechanics language a critical problem is decomposed into a sequence of subcritical ones. Here a critical problem is one where fluctuations of the dynamical variables over vastly different length scales have to be taken into account; for a subcritical problem the opposite is true. In more detail, let $\mathcal{O}$ be a function of the fields $\chi$ whose functional average is meant to be a macroscopic observable, but whose statistical average is sensitive to fluctuations of the microscopic fields $\chi$ on very different length scales. The replacement by a sequence of subcritical problems is done by specifying an asymmetric blocking kernel $K$ : Configurations $\times$ Configurations $\rightarrow \mathbb{R}, K\left(\chi^{\prime}, \chi\right)=K\left(\left\{\chi_{p}^{\prime}\right\},\left(\left\{\chi_{p}\right\}\right)\right.$, such that

(a) $K\left(\chi^{\prime}, \chi\right)=K_{l, \delta l}\left(\chi^{\prime}, \chi\right)$ has support mostly on configurations $\left\{\chi_{p}\right\}$ with $l-\delta l \leq$ $p \leq l$.

(b) $\int \prod_{p \leq \Lambda} d \chi_{p}^{\prime} K\left(\chi^{\prime}, \chi\right)=1$.

Then

$$
\langle\mathcal{O}\rangle=\int \prod_{p \leq \Lambda} d \chi_{p} \mathcal{O}(\chi) e^{-S[\chi]}=\int \prod_{p \leq \Lambda-\delta l} d \chi_{p}^{\prime} \mathcal{O}^{\prime}\left(\chi^{\prime}\right) e^{-S^{\prime}\left[\chi^{\prime}\right]}
$$

with

$$
\mathcal{O}^{\prime}\left(\chi^{\prime}\right) e^{-S^{\prime}\left[\chi^{\prime}\right]}=\int \prod_{p \leq \Lambda} d \chi_{p} K_{\Lambda, \delta l}\left(\chi^{\prime}, \chi\right) \mathcal{O}(\chi) e^{-S[\chi]} .
$$

Taking $\mathcal{O}=\mathbb{1}$ defines the coarse grained action functional $S^{\prime}$, after which (A.2) can be used to define the coarse grained observables $\mathcal{O}^{\prime}$. Property (a) entails that only field configurations with a similar 'degree of roughness' have to be considered in evaluating the functional integral in (A.2). It should thus be much more amenable to (numerical or analytical) approximation techniques than the original functional integral (A.1).

Once (A.2) has been evaluated one can iterate the procedure. The formulas (A.1), (A.2) remain valid with the basic kernel $K$ replaced by its $n$-fold convolution product, for which we write $K_{\Lambda, n \delta l}\left(\chi^{\prime}, \chi\right)$. For most choices a kernel $K_{l, \delta l}$ will not be reproducing, i.e. $\int \prod_{p \leq \Lambda} d \chi_{p}^{\prime} K_{l-\delta l, \delta l}\left(\chi^{\prime \prime}, \chi^{\prime}\right) K_{l, \delta l}\left(\chi^{\prime}, \chi\right)=: K_{l-2 \delta l, 2 \delta l}\left(\chi^{\prime \prime}, \chi\right)$ will not (despite the suggestive notation) coincide with the original kernel $K_{l, \delta l}$, just with modified parameters. Technically it is thus easier to specify the iterated kernel directly, which is of course still normalized. The $n$-fold iterated kernel will have support mostly on configurations 
with $e^{-t}:=\frac{l}{\Lambda} \leq \frac{p}{\Lambda} \leq 1$, if $l=n \delta l$, and $e^{-t}$ is the fraction of the momentum modes over which the functional integral has been performed after $n$ iterations. In the above terminology the critical problem (A.1) has been replaced by the sequence of subcritical problems (A.2). In each iteration, referred to as a coarse graining step defined by the kernel $K$, only a small fraction of the degrees of freedom is integrated out. The action $S=S_{\Lambda}$ at the cutoff scale $p=\Lambda$ is called the microsocopic (or bare) action, the $S^{\prime}=S_{l}$ reached after integrating out the 'fast' modes in the range $l / \Lambda \leq p / \Lambda \leq 1$ is called the coarse grained action at scale $l$, and similarly for the fields $\chi^{\prime}=\chi_{l}$. Note that the action $S_{l}[\cdot]$ as a functional is defined for all field configurations though for the evaluation of (A.1) only $S_{l}\left[\chi_{l}\right]$ is needed.

Throughout we shall follow the sloppy field theory convention that the coarse graining operates on the action. Of course what really gets updated is the functional measure

$$
d \mu_{l}[\chi]=\prod_{p} d \chi_{p} e^{-S_{l}[\chi]}
$$

In the (lattice) regularized theory the decomposition of the measure into a flat reference measure $\prod_{p} d \chi_{p}$ and a Boltzmann factor parameterized by the action is unproblematic. The flow in the measures can thus be traded for a flow in the actions (as long as the Jacobian is taken into account that comes from the reference measure upon a change of field variables $\left.\chi \mapsto \chi^{\prime}(\chi)\right)$. The Wilsonian "space of actions" refers to a cone of positive measures (A.3) which is preserved under the coarse graining operation considered.

In a gravitational context the very concept of renormalizabilty is less clear cut, and one should presumably go back to the even more fundamental property for which renormalizability is believed to be instrumental, namely the existence of a genuine continuum limit, roughly in the sense outlined in Section 1.3. Since rigorous results based on controlled approximations are unlikely to be obtained in the near future, we describe in the following criteria for the plausible existence of a genuine continuum limit based on two uncontrolled approximations: renormalized perturbation theory and the functional renormalization group approach. Such criteria are 'implicit wisdom' and are hardly ever spelled out. In the context of Quantum Gravidynamics, however, the absence of an obvi-

ous counterpart of the correlation length and the lack of perturbative renormalizability makes things more subtle. In the two appendices below we therefore try to make the implicit explicit and to formulate critera for the existence of a genuine continuum limit which are applicable to Quantum Gravidynamics as well.

\section{A.1 Perturbation theory and continuum limit}

Perturbatively renormalizable field theories are a degenerate special case of the WilsonKadanoff framework. The main advantage of perturbation theory is that the UV cutoff $\Lambda$ can be removed exactly and independently of the properties of the coupling flow. 
The existence of a $\Lambda \rightarrow \infty$ limit with the required properties (PTC1) can often be rigorously proven, in contrast to most nonperturbative techniques where this can only be established approximately by assembling evidence. With (PTC1) satisfied, the coupling flow then can be studied in a second step and used to probe whether or not the criterion (PTC2) for the existence of a genuine continuum limit as anticipated in Section 1.4 is also satisfied. The main disadvantage of perturbation theory is that everything is initially defined as a formal power series in the loop counting parameter. Even if one trades the latter for a running coupling the series in this coupling remains a formal one, typically non-convergent and not Borel-summable. It is generally believed, however, that provided (PTC2) is satisfied for a perturbative Gaussian fixed point, the series is asymptotic to the (usually unknown) exact result. In this case the perturbative analysis should indicate the existence of a genuine continuum limit based on an underlying Gaussian fixed point proper. Our main reason for going through this in some detail below is to point out that in a situation with several couplings the very same rationale applies if the perturbative fixed point is a non-Gaussian rather than a Gaussian one.

As mentioned, in perturbation theory one initially only aims at defining the expectations (A.1) as a formal powerseries in the loop counting parameter $\lambda$. In the regularized functional integral $\lambda$ enters via $\exp \left\{-\frac{1}{\lambda} S_{\Lambda}[\chi]\right\}$ and the (bare) perturbative expansion is a saddle point expansion around $\lambda=0$. The fluctuations around a saddle point configuration are rescaled by a factor $\lambda^{1 / 2}$ after which the sum of all $\ell$-loop contributions to a quantity occurs with a factor $\lambda^{\ell}$. The quadratic part of the expansion defines the propagators of a set of free fields; for definiteness we consider here the case where these are formally massless. For the reasons explained in [77] the loop expansion then does not necessarily coincide with an expansion in powers of Planck's constant $\hbar$. For example 1-loop diagrams can contribute to the classical limit $O\left(\hbar^{0}\right)$. Typically the field are given a reference mass $\mu$ and we write $S_{*, \mu}[\chi]$ for the (quadratic) action of this set of free fields. The interaction is described by a set of monomials $P_{i}[\chi], i \in E_{\mathrm{p} . c .}$, which are "powercounting renormalizable". The latter means that their mass dimension $-d_{i}$ is such that $d_{i} \geq 0$. It is also assumed that the $P_{i}$ are functionally independent, so that the corresponding couplings are essential. The so-called "bare" action functional then is $S_{\Lambda}=S_{*, \mu}+\sum_{i \in E_{\mathrm{p} . c .}} u_{i}(\Lambda) P_{i}$, where $u_{i}(\Lambda)$ are the essential "bare" couplings (including masses) corresponding to the interaction monomials $P_{i}[\chi]$. Inessential parameters are generated by subjecting $S_{\Lambda}$ to a suitable class of field redefinitions. In more detail one writes

$$
\begin{aligned}
u_{i}(\Lambda) & =u_{i}(\mu) V_{i, 0}(\mu)+\sum_{\ell \geq 1} \lambda^{\ell} V_{i, \ell}(u(\mu), \Lambda, \mu) \\
\chi_{\Lambda} & =\chi_{\mu}+\sum_{\ell \geq 1} \lambda^{\ell} \Xi_{\ell}\left(\chi_{\mu} ; u(\mu), \Lambda, \mu\right) .
\end{aligned}
$$

Here $u_{i}(\mu)$ are the renormalized couplings which are $\Lambda$ independent and the $V_{i}(u(\mu), \Lambda, \mu)$ are counterterms which diverge in the limit $\Lambda \rightarrow \infty$. This divergence is enforced by very general properties of QFTs. Similarly the $\chi_{\mu}$ are called renormalized fields and the 
$\Xi_{\ell}\left(\chi_{\mu} ; u(\mu), \Lambda, \mu\right)$ are local functionals of the $\chi_{\mu}$ with coefficients depending on $u(\mu), \Lambda, \mu$; the coefficients again diverge in the limit $\Lambda \rightarrow \infty$. Often one aims at "multiplicative renormalizability", which means the ansatz for the $\Xi_{\ell}$ is taken to be linear in the fields $\Xi_{\ell}\left(\chi_{\mu} ; u(\mu), \Lambda, \mu\right)=Z_{\ell}(u(\mu), \Lambda, \mu) \chi_{\mu}$ and $Z_{\ell}$ is the $\ell$-loop "wave function renormalization" constant. One should emphasize, however, that multiplicative renormalizability can often not be achieved, and even in field theories where it can be achieved, it evidently will work only with a particular choice of field coordinates; see [29] for a discussion.

The normalizations in (A.4) can be chosen such that $u_{i}(\mu=\Lambda)=u_{i}(\Lambda)$ and $\chi_{\mu=\Lambda}=\chi_{\Lambda}$, but one is really interested in the regime where $\mu \ll \Lambda$. Inserting these parameterizations into $S_{\Lambda}\left[\chi_{\Lambda}\right]$ gives an expression of the form

$$
S_{\Lambda}\left[\chi_{\Lambda}\right]=S_{*, \mu}\left[\chi_{\mu}\right]+\sum_{\alpha}\left(\sum_{\ell \geq 0} \lambda^{\ell} u_{\alpha, \ell}(u(\mu), \Lambda, \mu)\right) P_{\alpha}\left[\chi_{\mu}\right]
$$

where the sum over $\alpha$ includes terms of a form which can be absorbed by a nonlinear field redefinition, see e.g. Appendix A of [110]. Often the $\mu$-dependence in the fields can be traded for one carried by (inessential) parameters $z_{i}(\mu), i \in I$. Then (A.5) takes the form $S_{\Lambda}\left[\chi_{\Lambda}\right]=\sum_{\alpha^{\prime}} u_{\alpha^{\prime}}(\mathrm{g}(\mu), z(\mu), \Lambda, \mu) P_{\alpha^{\prime}}[\chi]$, with some $\mu$-independent fields, $\chi=\chi_{\mu_{0}}$, say. The rhs clearly resembles the Wilsonian form $\sum_{\alpha} u_{\alpha} P_{\alpha}$, with the difference that modulo field redefinitions only powercounting renormalizable interaction monomials occur.

So far the counterterms in (A.4) have been left unspecified. The point of introducing them is of course as a means to absorb the cut-off dependence generated by the regularized functional integral in (A.1). Specifically, one replaces the Boltzmann factor by its power series expansion in $\lambda$, i.e. $\exp \left\{-S_{\Lambda}\left[\chi_{\Lambda}\right]\right\}=\exp \left\{-S_{*, \mu}\left[\chi_{\mu}\right]\right\}\left(1+\sum_{\ell \geq 1} \lambda^{\ell} Q_{\ell}\left[\chi_{\mu}\right]\right)$, and aims at an evaluation of multipoint functions $\left\langle\chi_{\Lambda}\left(x_{1}\right) \ldots \chi_{\Lambda}\left(x_{n}\right)\right\rangle_{S_{\Lambda}}$ as formal power series in $\lambda$. After inserting (A.4) and the expansion of $e^{-S_{\Lambda}\left[\chi_{\Lambda}\right]}$ this reduces the problem to an evaluation of the free multipoint functions $\left\langle\chi_{\mu}\left(x_{1}\right) \ldots \chi_{\mu}\left(x_{n}\right) Q_{l}\left[\chi_{\mu}\right]\right\rangle_{S_{*, \mu}}$ computed with the quadratic action $S_{*, \mu}$ on the field space with cutoff $\Lambda$. The free multipoint functions will contain contributions which diverge in the limit $\Lambda \rightarrow \infty$. On the other hand via the parameterization (A.4),(A.5) the coefficients carry an adjustable $\Lambda$ dependence. In a renormalizable QFT the $\Lambda$ dependence in the coefficients can be chosen so as to cancel (for $\mu \ll \Lambda$ ) that generated by the multipoint functions $\left\langle\chi_{\mu}\left(x_{1}\right) \ldots \chi_{\mu}\left(x_{n}\right) Q_{l}\left[\chi_{\mu}\right]\right\rangle_{S_{*, \mu}}$. With this adjustment the limits

$$
\sum_{\ell \geq 0} \lambda^{\ell} \lim _{\Lambda \rightarrow \infty}\left\langle\chi_{\Lambda}\left(x_{1}\right) \ldots \chi_{\Lambda}\left(x_{n}\right)\right\rangle_{S_{\Lambda}, \ell}=: \sum_{\ell \geq 0} \lambda^{\ell}\left\langle\chi_{\mu}\left(x_{1}\right) \ldots \chi_{\mu}\left(x_{n}\right)\right\rangle_{S_{\mu}, \ell}
$$

exist and define the renormalized multipoint functions. As indicated they can be interpreted as referring to the renormalized action $\lim _{\Lambda \rightarrow \infty} S_{\Lambda}\left[\chi_{\Lambda}\right]=S_{\mu}\left[\chi_{\mu}\right]$. Eq. (A.6) highlights the main advantage of renormalized perturbation theory: the existence of the infinite cutoff limit (A.6) is often a provable property of the system, while this is not the case for most nonperturbative techniques. In the terminology introduced in Section 
1.3 the criterion (PTC1) is then satisfied. In order for this to be indicative for the existence of a genuine continuum limit, however, the additional condition (PTC2) must be satisfied, whose rationale we proceed to discuss now.

Since the renormalization scale $\mu$ is arbitrary, changing its value must not affect the values of observables. The impact of a change in $\mu$ can most readily be determined from (A.4). The left hand sides are $\mu$ independent, so by differentiating these relations with respect to $\mu$ and extracting the coefficients in a powerseries in (say) $\Lambda$ and/or $\log \Lambda$ consistency conditions arise for the derivatives $\mu \frac{d}{d \mu} u_{i}$ and $\mu \frac{d}{d \mu} \chi_{\mu}$. The ones obtained from the leading order are the most interesting relations. For the couplings one obtains a system of ordinary differential equations which define their renormalization flow under a change of $\mu$. As usual it is convenient to work with dimensionless couplings $g_{i}:=u_{i} \mu^{-d_{i}}$, where $d_{i}$ is the mass dimension of $u_{i}$. The flow equations then take the form

$$
\mu \frac{d}{d \mu} \mathrm{g}_{i}=\beta_{i}(\mathrm{~g}(\mu))
$$

where the $\beta_{i}$ are the perturbative beta functions. The flow equations for the renormalized fields are familiar only in the case of multiplicatively renormalizable fields, where one can work with scale independent fields and have the scale dependence carried by the wave function renormalization constant. In general however the fields are scale dependent. For example this ensures that the renormalized action evaluated on the renormalized fields is scale independent: $\mu \frac{d}{d \mu} S_{\mu}\left[\chi_{\mu}\right]=0$.

By construction the perturbative beta functions have a fixed point at $\mathrm{g}_{i}^{*}=0$, which is called the perturbative Gaussian fixed point. Nothing prevents them from having other fixed points, but the Gaussian one is built into the construction. This is because a free theory has vanishing beta functions and the couplings $\mathrm{g}_{i}=u_{i} \mu^{-d_{i}}$ have been introduced to parameterize the deviations from the free theory with action $S_{*, \mu}$. Not surprisingly the stability matrix $\Theta_{i j}=\partial \beta_{i} /\left.\partial \mathrm{g}_{j}\right|_{\mathrm{g}^{*}=0}$ of the perturbative Gaussian fixed point just reproduces the information which has been put in. The eigenvalues come out to be $-d_{i}$ modulo corrections in the loop coupling parameter, where $-d_{i}$ are the mass dimensions of the corresponding interaction monomials. For the eigenvectors one finds a one-toone correspondence to the unit vectors in the 'coupling direction' $\mathrm{g}_{i}$, again with power corrections in the loop counting parameter. One sees that the couplings $u_{i}$ not irrelevant with respect to the stability matrix $\Theta$ computed at the perturbative Gaussian fixed point are the ones with mass dimensions $d_{i} \geq 0$, i.e. just the power counting renormalizable ones.

The attribute "perturbative Gaussian" indicates that whenever in a nonperturbative construction of the renormalization flow in the same 'basis' of interaction monomials $\mathrm{g}_{i}^{*}=0$ is also a fixed point (called the Gaussian fixed point) the perturbatively defined expectations are believed to provide an asymptotic (nonconvergent) expansion to the 
expectations defined nonperturbatively based on the Gaussian fixed point, schematically

$$
\langle\mathcal{O}\rangle_{\text {GaussianFP }} \sim \sum_{\ell \geq 0} \lambda^{\ell}\langle\mathcal{O}\rangle_{\ell}
$$

Here $\langle\mathcal{O}\rangle_{\ell}$ is the perturbatively computed $\ell$-loop contribution after a so-called renormalization group improvement. Roughly speaking the latter amounts to the following procedure: one assigns to the loop counting parameter $\lambda$ a numerical value (ultimately related to the value of Planck's constant in the chosen units; see however [77]) and solves $\mu \frac{d \mathrm{~g}_{i}}{d \mu}=\beta_{i}(\mathrm{~g}(\mu))$ as an ordinary differential equation. One of the functions obtained, say $\mathrm{g}_{1}(\mu)$, is used to eliminate $\lambda$ in favor of $\mu$ and an integration constant $\Lambda_{\text {beta }}$ (not to be confused with the cutoff, which is gone for good). So $\langle\mathcal{O}\rangle_{L}:=\sum_{\ell \leq L} \bar{\lambda}\left(\mu, \Lambda_{\text {beta }}\right)^{\ell}\langle\mathcal{O}\rangle_{\ell, \mu}$ at this point carries a two-fold $\mu$-dependence, the one which comes out of the renormalization procedure (A.6) and the one carried now by $\bar{\lambda}\left(\mu, \Lambda_{\text {beta }}\right)$. For an observable quantity $\mathcal{O}$ both dependencies cancel out, modulo terms of higher order, leaving behind a dependence on the integration constant $\Lambda_{\text {beta }}$. We write $\langle\mathcal{O}\rangle_{L}\left(\Lambda_{\text {beta }}\right)$ to indicate this dependence. One then uses the expectation of one, suitably chosen, observable $\mathcal{O}_{0}$ to match its value $\left\langle\mathcal{O}_{0}\right\rangle$ (measured or otherwise known) with that of $\left\langle\mathcal{O}_{0}\right\rangle_{L}\left(\Lambda_{\text {beta }}\right)$ to a given small loop order $L$ (typically not larger than 2 ). For a well chosen $\mathcal{O}_{0}$ this allows one to replace $\Lambda_{\text {beta }}$ by a physical mass scale $m_{\text {phys }}$. Eliminating $\Lambda_{\text {beta }}$ in favor of $m_{\text {phys }}$ gives the perturbative predictions for all other observables. Apart from residual scheme dependences (which are believed to be numerically small) this defines the right hand side of (A.8) unambigously as a functional over the observables.

Nevertheless, except for some special cases, it is difficult to give a mathematically precise meaning to the ' $\sim$ ' in Equation (A.8). Ideally one would be able to prove that perturbation theory is asymptotic to the (usually unknown) exact answer for the same quantity. For lattice theories on a finite lattice this is often possible, the problems start when taking the limit of infinite lattice size, see [112] for a discussion. In the continuum limit a proof that perturbation theory is asymptotic has been achieved in a number of low dimensional quantum field theories: the superrenormalizable $P_{2}(\phi)$ and $\phi_{3}^{4}$ theories [47, 31] and the two-dimensional Gross-Neveu model, where the correlation functions are the Borel sum of their renormalized perturbation expansion [62, 63]. Strong evidence for the asymptotic correctness of perturbation theory has also been obtained in the $\mathrm{O}(3)$ nonlinear sigma-model via the form factor bootstrap [17]. In four or higher dimensional theories unfortunately no such results are available. It is still believed that whenever the above $g_{1}$ is asymptotically free in perturbation theory, that the corresponding series is asymptotic to the unknown exact answer. On the other hand, to the best of our knowledge, a serious attempt to establish the asymptotic nature of the expansion has never been made, nor are plausible strategies available. The pragmatic attitude usually adopted is to refrain from the attempt to theoretically understand the domain of applicability of perturbation theory. Instead one interprets the ' $\sim$ ' in (A.8) as approximate numerical equality, to a suitable loop order $L$ and in a benign scheme, 
as long as it works, and attributes larger discrepancies to the 'onset of nonperturbative physics'. This is clearly unsatisfactory, but often the best one can do. Note also that some of the predictive power of the QFT considered is wasted by this procedure and that it amounts to a partial immunization of perturbative predictions against (experimental or theoretical) refutation.

So far the discussion was independent of the nature of the running of $\bar{\lambda}\left(\mu, \Lambda_{\text {beta }}\right.$ ) (which was traded for $\mathrm{g}_{1}$ ). The chances that the vage approximate relation ' $\sim$ ' in (A.8) can be promoted to the status of an asymptotic expansion are of course way better if $\bar{\lambda}\left(\mu, \Lambda_{\text {beta }}\right)$ is driven towards $\bar{\lambda}=0$ by the perturbative flow. Only then is it reasonable to expect that an asymptotic relation of the form (A.8) holds, linking the perturbative Gaussian fixed point to a genuine Gaussian fixed point defined by nonperturbative means. The perturbatively and the nonperturbatively defined coupling $\mathrm{g}_{1}$ can then be identified asymptotically and lie in the unstable manifold of the fixed point $\mathrm{g}_{1}^{*}=0$. On the other hand the existence of a Gaussian fixed point with a nontrivial unstable manifold is thought to entail the existence of a genuine continuum limit, in the sense discussed before. In summary, if $\mathrm{g}_{1}$ is traded for a running $\bar{\lambda}\left(\mu, \Lambda_{\text {beta }}\right)$, a perturbative criterion for the existence of a genuine continuum limit is that the perturbative flow of $g_{1}$ is regular with $\lim _{\mu \rightarrow \infty} \mathrm{g}_{1}(\mu)=0$. Since the beta functions of the other couplings are formal power series in $\lambda$ without constant coefficients the other couplings will vanish likewise as $\mathrm{g}_{1} \rightarrow 0$, and one recovers the local quadratic action $S_{*, \mu}[\chi]$ at the fixed point. The upshot is that the coupling with respect to which the perturbative expansion is performed should be asymptotically free in perturbation theory in order to render the existence of a nonperturbative continuum limit plausible.

The reason for going through this discussion is to highlight that is applies just as well to a perturbative non-Gaussian fixed point. This sounds like a contradiction in terms, but it isn't. Suppose that in a situation with several couplings $g_{1}, \ldots, g_{n}$ the perturbative beta functions (which are formal power series in $\lambda$ without constant coefficients) admit a nontrivial zero, $\mathrm{g}_{1}^{*}(\lambda), \ldots, \mathrm{g}_{n}^{*}(\lambda)$. Suppose in addition that all the couplings lie in the unstable manifold of that zero, i.e. the flows $\mathrm{g}_{i}(\mu)$ are regular and $\lim _{\mu \rightarrow \infty} \mathrm{g}_{i}(\mu)=\mathrm{g}_{i}^{*}$. We shall call a coupling with this property asymptotically safe, so that the additional assumption is that all couplings are asymptotically safe. As before one must assign $\lambda$ a numerical value in order to define the flow. Since the series in $\lambda$ anyhow has zero radius of convergence, the 'smallness' of $\lambda$ is not off-hand a measure for the reliability of the perturbative result (the latter intuition in fact precisely presupposes (A.8)). Any one of the deviations $\delta \mathrm{g}_{i}=\mathrm{g}_{i}-\mathrm{g}_{i}^{*}$, which is of order $\lambda$ at some $\mu$ can be used as well to parameterize the original loop expansion. By a relabeling or reparameterization of the couplings we may assume that this is the case for $\delta \mathrm{g}_{1}$. The original loop expansion can then be rearranged to read $\sum_{\ell \geq 0}\left(\delta \mathrm{g}_{1}\right)^{\ell}\langle\mathcal{O}\rangle_{\ell}$. However, if there is an underlying nonperturbative structure at all, it is reasonable to assume that it refers to a nonGaussian fixed point,

$$
\langle\mathcal{O}\rangle_{\text {Non-GaussianFP }} \sim \sum_{\ell \geq 0}\left(\delta \mathrm{g}_{1}\right)^{\ell}\langle\mathcal{O}\rangle_{\ell}
$$


The rationale for (A.9) is exactly the same as for (A.8). What matters is not the value of the couplings at a perturbative fixed point, but their flow pattern. For a nontrivial fixed point the couplings $\mathrm{g}_{i}^{*}$ in the above basis of interaction monomials are nonzero, but any one of the deviations $\delta \mathrm{g}_{i}=\mathrm{g}_{i}-\mathrm{g}_{i}^{*}$ can be made arbitrarily small as $\mu \rightarrow \infty$. The relation ' $\sim$ ' in (A.9) then again plausibly amounts to an asymptotic expansion for the unknown exact answer, where the latter this time is based on a non-Gaussian fixed point.

Summarizing: in perturbation theory the termwise removal of the UV cutoff can be achieved independently of the properties of the coupling flow, while in a non-perturbative setting both aspects are linked. Only if the coupling flow computed from the perturbative beta functions meets certain conditions is it reasonable to assume that there exists an underlying non-perturbative framework to whose results the perturbative series is asymptotic. Specifically we formulate the

Criterion: (Continuum limit via perturbation theory) (PTC1) Existence of a formal continuum limit, i.e. termwise removal of the UV cutoff is possible and the renormalized physical quantities are independent of the scheme and of the choice of interpolating fields, all in the sense of formal power series in the loop counting parameter. (PTC2) The perturbative beta functions have a Gaussian or a non-Gaussian fixed point and the dimension of its unstable manifold (as computed from the perturbative beta functions) equals the number of independent essential couplings. Equivalently, all essential couplings are asymptotically safe in perturbation theory.

\section{A.2 Functional flow equations and UV renormalization}

The technique of functional renormalization group equations (FRGEs) does not rely on a perturbative expansion and has been widely used for the computation of critical exponents and the flow of generalized couplings. For a systematic exposition of this technique and its applications we refer to the reviews $[103,116,151,20,102]$. Here we shall mainly use the effective average action $\Gamma_{k}$ and its 'exact' FRGE. We refer to [110] for a summary of this formulation, and discuss in this Section how the UV renormalization problem presents itself in an FRGE.

In typical applications of the FRG the ultraviolet renormalization problem does not have to be addressed. In the context of the asymptotic safety scenario this is different. By definition the perturbative series in a field theory based on an asymptotically safe functional measure has a dependence on the UV cutoff which is not strictly renormalizable, see Section 1.3. The perturbative expansion of an FRGE must reproduce the structure of these divergencies. On the other hand in an exact treatment or based on different approximation techniques a reshuffling of the cutoff dependence is meant to occur which allows for a genuine continuum limit. We therefore outline here how the UV renormalization problem manifests itself in the framework of the functional flow equations. The goal will be to formulate a criterion for the plausible existence of a genuine continuum 
limit in parallel to the one above based on perturbative indicators.

Again we illustrate the relevant issues for a scalar quantum field theory on flat space. For definiteness we consider here the flow equation for the effective average action $\Gamma_{\Lambda, k}[\phi]$, for other types of FRGEs the discussion is similar though. The effective average action interpolates between the bare action $S_{\Lambda}[\phi]$ and the above, initially regulated, effective action $\Gamma_{\Lambda}$, according to

$$
S_{\Lambda}[\phi] \stackrel{k \rightarrow \Lambda}{\longleftarrow} \Gamma_{\Lambda, k}[\phi] \stackrel{k \rightarrow 0}{\longrightarrow} \Gamma_{\Lambda}[\phi]
$$

Roughly speaking one should think of $\Gamma_{\Lambda, k}[\phi]$ as the conventional effective action but with only the momentum modes in the range $k^{2}<p^{2}<\Lambda^{2}$ integrated out. The $k \rightarrow \Lambda$ limit in (A.10) will in fact differ from $S_{\Lambda}$ by a 1-loop determinant $\ln \operatorname{det}\left[S_{\Lambda}^{(2)}+\mathcal{R}_{\Lambda}\right]$. For the following discussion the difference is inessential and for (notational) simplicity we will identify $\Gamma_{\Lambda, \Lambda}$ with $S_{\Lambda}$. Equation (A.10) also presupposes that for fixed UV cutoff $\Lambda$ the limit $k \rightarrow 0$ exists, which for theories with massless degrees of freedom is nontrivial.

The conventional effective action obeys a well-known functional integro-differential equation which implicitly defines it. Its counterpart for $\Gamma_{\Lambda, k}[\phi]$ reads

$$
\exp \left\{-\Gamma_{\Lambda, k}[\phi]\right\}=\int[\mathcal{D} \chi]_{\Lambda, k} \exp \left\{-S_{\Lambda}[\chi]+\int d x(\chi-\phi)(x) \frac{\delta \Gamma_{\Lambda, k}[\phi]}{\delta \phi(x)}\right\}
$$

where the functional measure $[\mathcal{D} \chi]_{\Lambda, k}$ includes mostly momentum modes in the range $k^{2}<p^{2}<\Lambda^{2}$. This can be done by multiplying the kinematical measure by a suitable mode suppression factor

$$
[\mathcal{D} \chi]_{\Lambda, k}=\mathcal{D} \chi \exp \left\{-C_{\Lambda, k}[\chi-\phi]\right\}
$$

with a suitable quadratic form $C_{\Lambda, k}$. From (A.11) one can directly verify the alternative characterization in Eq. (1.22).

The precise form of the mode suppression is inessential. In the following we outline a variant which is technically convenient. Here $C_{\Lambda, k}$ is a quadratic form in the fields defined in terms of a kernel $\mathcal{R}_{\Lambda, k}$ chosen such that both $\mathcal{R}_{\Lambda, k}$ and $k \partial_{k} \mathcal{R}_{\Lambda, k}$ define integral operators of trace-class on the function space considered. We write $\left[\mathcal{R}_{\Lambda, k} \chi\right](x):=$ $\int d y \mathcal{R}_{\Lambda, k}(x, y) \chi(y)$ for the integral operator and $\operatorname{Tr}\left[\mathcal{R}_{\Lambda, k}\right]:=\int d x \mathcal{R}_{\Lambda, k}(x, x)<\infty$ for its trace. The other properties of the kernel are best described in Fourier space, where $\mathcal{R}_{k, \Lambda}$ acts as $\left[\mathcal{R}_{\Lambda, k} \widehat{\chi}\right](p)=\int \frac{d q}{(2 \pi)^{d}} \mathcal{R}_{\Lambda, k}(p, q) \widehat{\chi}(q)$, with $\widehat{\chi}(p)=\int d x \chi(x) \exp (-i p x)$, the Fourier transform of $\chi$ and similarly for the kernel (where we omit the hat for notational simplicity). The UV cutoff $0 \leq p^{2}<\Lambda$ renders Euclidean momentum space compact and Mercer's theorem then provides simple sufficient conditions for an integral operator to be trace-class. We thus take the kernel $\mathcal{R}_{\Lambda, k}(p, q)$ to be smooth, symmetric in $p, q$, 
and such that

$$
C_{\Lambda, k}[\chi]:=\frac{1}{2} \int \frac{d p}{(2 \pi)^{d}} \frac{d q}{(2 \pi)^{d}} \widehat{\chi}(q)^{*} \mathcal{R}_{\Lambda, k}(p, q) \widehat{\chi}(p)=\frac{1}{2} \int d x \chi(x)^{*}\left[\mathcal{R}_{\Lambda, k} \chi\right](x) \geq 0
$$

for all continuous functions $\chi$. Similarly for $k \partial_{k} \mathcal{R}_{\Lambda, k}(p, q)$. The trace-class condition is then satisfied and one can adjust the other features of the kernel to account for the mode suppression. These features are arbitrary to some extent; what matters is the limiting behavior for $p^{2}, q^{2} \gg k^{2}$ and (with foresight) $\Lambda \rightarrow \infty$.

The presence of the extra scale $k$ allows one to convert (A.11) into a functional differential equation $[150,151,20]$,

$$
\begin{aligned}
k \frac{\partial}{\partial k} \Gamma_{\Lambda, k}[\phi] & =\frac{1}{2} \operatorname{Tr}\left[k \frac{\partial}{\partial k} \mathcal{R}_{\Lambda, k}\left(\Gamma_{\Lambda, k}^{(2)}[\phi]+\mathcal{R}_{\Lambda, k}\right)^{-1}\right] \\
& =\frac{1}{2} \int \frac{d q_{1}}{(2 \pi)^{d}} \frac{d q_{2}}{(2 \pi)^{d}} k \partial_{k} \mathcal{R}_{\Lambda, k}\left(q_{1}, q_{2}\right)\left(\Gamma_{\Lambda, k}^{(2)}[\phi]+\mathcal{R}_{\Lambda, k}\right)^{-1}\left(q_{2}, q_{1}\right) .
\end{aligned}
$$

known as the functional renormalization group equation (FRGE) for the effective average action. For convenience we included a quick derivation of (A.14) in Appendix C of [110]. In the second line of (A.14) we spelled out the trace using that $k \partial_{k} \mathcal{R}_{\Lambda, k}$ is traceclass. Further $\Gamma_{\Lambda, k}^{(2)}[\phi]$ is the integral operator whose kernel is the Hessian of the effective average action, i.e. $\Gamma_{\Lambda, k}^{(2)}(x, y):=\delta^{2} \Gamma_{\Lambda, k}[\phi] / \delta \phi(x) \delta \phi(y)$, and $\mathcal{R}_{\Lambda, k}$ is the integral operator in (A.11).

For finite cutoffs $(\Lambda, k)$ the trace of the right hand side of (A.14) will exist as the potentially problematic high momentum parts are cut off. In slightly more technical terms, since the product of a trace-class operator with a bounded operator is again trace-class, the trace in (A.14) is finite as long as the inverse of $\Gamma_{\Lambda, k}^{(2)}[\phi]+\mathcal{R}_{\Lambda, k}$ defines a bounded operator. For finite UV cutoff one sees from the momentum space version of it that this will normally be the case. The trace-class property of the mode cutoff operator (for which (A.13) is a sufficient condition) also ensures that the trace in (A.14) can be evaluated in any basis, the momentum space variant displayed in the second line is just one convenient choice.

Importantly the FRGE (A.14) is independent of the bare action $S_{\Lambda}$, which enters only via the initial condition $\Gamma_{\Lambda, \Lambda}=S_{\Lambda}$ (for large $\Lambda$ ). In the FRGE approach the calculation of the functional integral for $\Gamma_{\Lambda, k}$ is replaced with the task of integrating this RG equation from $k=\Lambda$, where the initial condition $\Gamma_{\Lambda, \Lambda}=S_{\Lambda}$ is imposed, down to $k=0$, where the effective average action equals the ordinary effective action $\Gamma_{\Lambda}$.

All this has been for a fixed UV cutoff $\Lambda$. The removal of the cutoff is of course the central theme of UV renormalization. In the FRG formulation one has to distinguish between two aspects. First, removal of the explicit $\Lambda$ dependence in the trace on the right hand side of (A.14), and second removal of the UV cutoff in $\Gamma_{\Lambda, k}$ itself, which was needed in order to make the original functional integral well-defined. 
The first aspect is unproblematic: the trace is manifestly finite as long as the inverse of $\Gamma_{\Lambda, k}^{(2)}[\phi]+\mathcal{R}_{\Lambda, k}$ defines a bounded operator. If now $\Gamma_{\Lambda, k}^{(2)}[\phi]$ is independently known to have a finite and nontrivial limit as $\Lambda \rightarrow \infty$, the explicit $\Lambda$ dependence carried by the $\mathcal{R}_{\Lambda, k}$ term is harmless and the trace always exists. Roughly this is because the derivative kernel $k \partial_{k} \mathcal{R}_{\Lambda, k}$ has support mostly on a thin shell around $p^{2} \approx k^{2}$, so that the (potentially problematic) large $p$ behavior of the other factor is irrelevant; cf. [20].

The second aspect of course relates to the traditional UV renormalization problem. Since $\Gamma_{\Lambda, k}$ came from a regularized functional integral it will develop the usual UV divergencies as one attempts to send $\Lambda$ to infinity. The remedy is to carefully adjust the bare action $S_{\Lambda}[\phi]$ - that is, the initial condition for the FRGE (A.14) - in such a way that functional integral - viz, the solution of the FRGE - is asymptotically independent of $\Lambda$. Concretely this could be done by fine-tuning the way how the parameters $u_{\alpha}(\Lambda)$ in the expansion $S_{\Lambda}[\chi]=\sum_{\alpha} u_{\alpha}(\Lambda) P_{\alpha}[\chi]$ depends on $\Lambda$. However the FRGE method in itself provides no means to find the proper initial functional $S_{\Lambda}[\chi]$. Identification of the fine-tuned $S_{\Lambda}[\chi]$ lies at the core of the UV renormalization problem, irrespective of whether $\Gamma_{\Lambda, k}$ is defined directly via the functional integral or via the FRGE. Beyond perturbation theory the only known techniques to identify the proper $S_{\Lambda}$ start directly from the functional integral and are 'constructive' in spirit. Unfortunately four dimensional quantum field theories of interest are still beyond constructive control.

One may also ask whether perhaps the cutoff-dependent FRGE (A.14) itself can be used to show that a limit $\lim _{\Lambda \rightarrow \infty} \Gamma_{\Lambda, k}[\phi]$ exists. Indeed using other FRGEs and a perturbative ansatz for the solution has lead to economic proofs of perturbative renormalizability, i.e. of the existence of a formal continuum limit in the sense (PTC1) discussed before. Unfortunately so far this could not be extended to construct a nonperturbative continuum limit of fully fledged quantum field theories, see [102] for a recent review of such constructive uses of FRGEs. For the time being one has to be content with the following if ... then statement:

If there exists a sequence of initial actions $S_{n \Lambda_{0}}[\chi], n \in \mathbb{N}$, such that the solution $\Gamma_{n \Lambda_{0}, k}[\phi]$ of the FRGE (A.14) remains finite as $n \rightarrow \infty$, then the limit $\Gamma_{k}[\phi]:=\lim _{n \rightarrow \infty} \Gamma_{n \Lambda_{0}, k}[\phi]$ has to obey the cut-off independent FRGE

$$
k \frac{\partial}{\partial k} \Gamma_{k}[\phi]=\frac{1}{2} \operatorname{Tr}\left[k \frac{\partial}{\partial k} \mathcal{R}_{k, \infty}\left(\Gamma_{k}^{(2)}[\phi]+\mathcal{R}_{k, \infty}\right)^{-1}\right] .
$$

Conversely, under the above premise, this equation should have at least one solution with

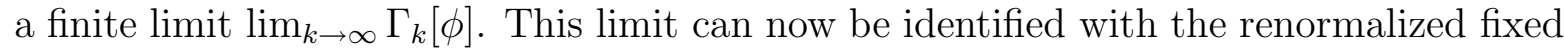
point action $S_{*}[\chi]$. It is renormalized because by construction the cutoff dependencies have been eaten up by the ones produced by the trace in (A.14). It can be identified with a fixed point action because lowering $k$ amounts to coarse graining and $S_{*}[\chi]$ is the 'inverse limit' of a sequence of such coarse graining steps.

So far the positivity or unitarity requirement has not been discussed. From the (Osterwalder-Schrader or Wightman) reconstruction theorems it is known how the unitarity 
of a quantum field theory on a flat spacetime translates into nonlinear conditions on the multipoint functions. Since the latter can be expressed in terms of the functional derivatives of $\Gamma_{k}$, unitarity can in principle be tested retroactively, and is expected to hold only in the limit $k \rightarrow 0$. Unfortunately this is a very indirect and retroactive criterion. One of the roles of the bare action $S_{\Lambda}[\chi]=\Gamma_{\Lambda, \Lambda}[\chi]$ is to encode properties which are likely to ensure the desired properties of $\lim _{k \rightarrow 0} \Gamma_{k}[\phi]$. In theories with massless degrees of freedom the $k \rightarrow 0$ limit is nontrivial and the problem of gaining computational control over the infrared physics should be separated from the UV aspects of the continuum limit as much as possible. However the $k \rightarrow 0$ limit is essential to probe stability/positivity/unitarity.

One aspect of positivity is the convexity of the effective action. The functional equations (A.14) and (A.15) do in itself "not know" that $\Gamma_{k}$ is the Legendre transform of a convex functional and hence must be itself convex. Convexity must therefore enter through the inital data and it will also put constraints on the choice of the mode cutoffs. Good mode cutoffs are characterized by the fact that $\Gamma_{k}^{(2)}+\mathcal{R}_{k}$ has positive spectral values for all $k$. If no blow-up occurs in the flow the $\operatorname{limit}_{\lim } \rightarrow 0 \Gamma_{k}^{(2)}$ will then also have non-negative spectrum. Of course this presupposes again that the proper initial conditions have been identified and the role of the bare action is as above.

For flat space quantum field theories one expects that $S_{\Lambda}[\chi]$ must be local, i.e. a differential polynomial of finite order in the fields so as to end up with an effective action $\lim _{k \rightarrow \infty} \lim _{\Lambda \rightarrow \infty} \Gamma_{\Lambda, k}[\phi]$ describing a local/microcausal unitary quantum field theory.

For convenient reference we summarize these conclusions in the

Criterion: (Continuum limit in the functional RG approach) (FRGC1) A solution of the cutoff independent FRGE (A.15) which exists globally in $k$ (for all $0 \leq k \leq$ $\infty)$ can reasonably be identified with the continuum limit of the effective average action $\lim _{\Lambda \rightarrow \infty} \Gamma_{\Lambda, k}[\phi]$ constructed by other means. For such a solution $\lim _{k \rightarrow 0} \Gamma_{k}[\phi]$ is the full quantum effective action and $\lim _{k \rightarrow \infty} \Gamma_{k}[\phi]=S_{*}[\phi]$ is the fixed point action. (FRGC2) For a unitary relativistic quantum field theory positivity/unitarity must be tested retroactively from the functional derivatives of $\lim _{k \rightarrow 0} \Gamma_{k}[\phi]$.

We add some comments:

Since the FRGE (A.15) is a differential equation in $k$, an initial functional $\Gamma_{\text {initial }}[\phi]$ has to be specified for some $0<k_{\text {initial }} \leq \infty$, to generate a local solution near $k=k_{\text {initial }}$. The point is that for 'almost all' choices of $\Gamma_{\text {initial }}[\phi]$ the local solution cannot be extended to all values of $k$. Finding the rare initial functionals for which this is possible is the FRGE counterpart of the UV renormalization problem. The existence of the $k \rightarrow 0$ limit is itself not part of the UV problem, in conventional quantum field theories the $k \rightarrow 0$ limit is however essential to probe unitarity/positivity/stability.

It is presently not known whether the above criterion can be converted into a theorem. Suppose for a quantum field theory on the lattice (with lattice spacing $\Lambda^{-1}$ ) the effective action $\Gamma_{\Lambda, k}^{\text {latt }}[\phi]$ has been constructed nonperturbatively from a transfer operator satisfying 


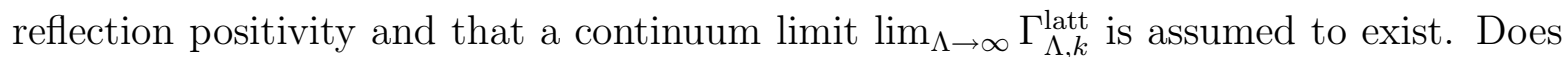
it coincide with a solution $\Gamma_{k}[\phi]$ of (A.15) satisfying the criteria (FRGC1), (FRGC2)? Note that this is 'only' a matter of controlling the limit, for finite $\Lambda$ also $\Gamma_{\Lambda, k}^{\text {latt }}$ will satisfy the flow equation (A.14).

For an application to quantum gravity one will initially only ask for (FRGC1), perhaps with even only a partial understanding of the $k \rightarrow 0$ limit. As mentioned, the $k \rightarrow 0$ limit should also be related to positivity issues. The proper positivity requirement replacing (FRGC2) yet has to be found, however some constraint will certainly be needed. Concerning (FRGC1) the premise in the if ... then statement preceeding (A.15) has to be justified by external means or taken as a working hypothesis. In principle one can also adopt the viewpoint that the quantum gravity counterpart of (A.15) introduced in [127] simply defines the effective action for quantum gravity whenever a solution meets (FRGC1). The main drawbacks of this proposal are: first, beyond perturbation theory little is known about the structure of $\Gamma_{k}$, e.g. which type of nonlocalities are expected to occur. Second, the proposal would make it difficult to include information concerning (FRGC2). However difficult and roundabout a functional integral construction is, it allows one to incorporate 'other' desirable features of the system in a relatively transparent way.

Also in the application to quantum gravity we shall therefore presuppose that a solution $\Gamma_{k}$ of the cutoff independent FRGE (A.15) satisfying (FRGC1) comes from an underlying functional integral. This amounts to the assumption that the renormalization problem for $\Gamma_{k, \Lambda}$ defined in terms of a functional integral can be solved and that the limit $\lim _{\Lambda \rightarrow \infty} \Gamma_{k, \Lambda}$ can be identified with $\Gamma_{k}$. This is of course a rather strong hypothesis, however its selfconsistency can be tested within the FRG framework.

To this end one truncates the space of candidate continuum functionals $\Gamma_{k}^{\mathrm{trunc}}[\phi]$ to one where the initial value problem for the flow equation (A.15) can be solved in reasonably closed form. One can then by 'direct inspection' determine the initial data for which a global solution exists. Convexity of the truncated $\lim _{k \rightarrow 0} \Gamma_{k}^{\operatorname{trunc}}[\phi]$ can serve as guideline to identify good truncations. If the set of these initial data forms a nontrivial unstable manifold of the fixed point $S_{*}^{\text {trunc }}[\phi]=\lim _{k \rightarrow \infty} \Gamma_{k}^{\text {trunc }}[\phi]$ application of the above criterion suggests that $\Gamma_{k}^{\text {trunc }}$ can approximately be identified with the projection of the continuum limit $\left(\lim _{\Lambda \rightarrow \infty} \Gamma_{\Lambda, k}\right)^{\text {trunc }}$ of some $\Gamma_{\Lambda, k}$ computed by other means. The identification can only be an approximate one because in the $\Gamma_{k}^{\text {trunc }}$ evolution one first truncates and then evolves in $k$, while in $\left(\lim _{\Lambda \rightarrow \infty} \Gamma_{\Lambda, k}[\phi]\right)^{\text {trunc }}$ one first evolves in $k$ and then truncates. Alternatively one can imagine to have replaced the original dynamics by some 'hierarchical' approximation implicitly defined by the property that $\left(\lim _{\Lambda \rightarrow \infty} \Gamma_{\Lambda, k}^{\text {hier }}[\phi]\right)^{\text {trunc }}=\lim _{\Lambda \rightarrow \infty} \Gamma_{\Lambda, k}^{\text {hier }}[\phi]$. (See [52]) for the relation between hierachical dynamics and the local potential approximation). The existence of an UV fixed point with a nontrivial unstable manifold for $\Gamma_{k}^{\text {trunc }}$ can then be taken as witnessing the renormalizability of the 'hierarchical' dynamics. 


\section{References}

[1] L. Abott, The background field method beyond one loop, Nucl. Phys. B 185 (1981) 189.

[2] A. Adams, N. Arkani-Hamed, S. Dubovsky, A. Nicolis and R. Rattazzi, Causality, analyticity and an IR obstruction to UV completion, JHEP 0610 (2006) 014; [arXiv:hepth/0602178].

[3] T. Aida, Y. Kitazawa, H. Kawai and M. Ninomiya, Conformal invariance and renormalization group in quantum gravity near two-dimensions, Nucl. Phys. B 427, 158 (1994) [arXiv:hep-th/9404171].

[4] J. Ambjorn, J. Jurkiewicz and R. Loll, Dynamically triangulating Lorentzian quantum gravity, Nucl. Phys. B 610, 347 (2001) [arXiv:hep-th/0105267].

[5] J. Ambjorn, J. Jurkiewicz and Y. Watabiki, Dynamical triangulations, a gateway to quantum gravity?, J. Math. Phys. 36, 6299 (1995) [arXiv:hep-th/9503108].

[6] J. Ambjorn, J. Jurkiewicz and R. Loll, Spectral dimension of the universe, Phys. Rev. Lett. 95, 171301 (2005) [arXiv:hep-th/0505113].

[7] I. Anderson and C. Torre, Classification of generalized symmetries for the vacuum Einstein equations, Commun. Math. Phys. 176, 479 (1996) [arXiv:gr-qc/9404030].

[8] D. Anselmi, Absence of higher derivatives in the renormalization of propagators in quantum field theories with infinitely many couplings, Class. Quant. Grav. 20 (2003) 23552378; [arXiv:hep-th/0212013].

[9] D. Anselmi, Infinite reduction of couplings in non-renormalizable quantum field theory, JHEP 0508, 029 (2005) [arXiv:hep-th/0503131].

[10] I. Antoniadis, P. Mazur and E. Mottola, Cosmological dark energy: Prospects for a dynamical theory, New J. Phys. 9, 11 (2007) [arXiv:gr-qc/0612068].

[11] I. Antoniadis and E. Mottola, Four dimensional quantum gravity in the conformal sector, Phys. Rev D45 (1992) 2013.

[12] I. Antoniadis, E. Dudas, and D. Ghilencea, Living with ghosts and their radiative corrections, Nucl. Phys. B767 (2007) 29; [arXiv:hep-th/0608094].

[13] M. Atance and J. Cortes, Effective field theory of gravity, reduction of couplings and the renormalization group, Phys. Rev. D 54, 4973 (1996) [arXiv:hep-ph/9605455].

[14] I. Avramidi, Heat kernel and quantum gravity, Lecture Notes in Physics, Springer 2006.

[15] I. Avramidi and A. Barvinsky, Asymptotic freedom in higher derivative quantum gravity, Phys. Lett. B 159, 269 (1985). 
[16] R. Balbinot, A. Fabbri and I. L. Shapiro, Vacuum polarization in Schwarzschild spacetime by anomaly induced effective actions, Nucl. Phys. B 559, 301 (1999) [arXiv:hepth/9904162].

[17] J. Balog and M. Niedermaier, Off-shell dynamics of the O(3) NLS model beyond Monte Carlo and perturbation theory, Nucl. Phys. B 500, 421 (1997) [arXiv:hep-th/9612039].

[18] V. Belinski and E. Verdaguer, Gravitational solitons, Cambridge Univ. Press, 2001.

[19] D. Ben-Avraham and S. Havlin, Diffusion and reactions in fractals and disordered systems, Cambridge Univ. Press, 2000.

[20] J. Berges, N. Tetradis and C. Wetterich, Non-perturbative renormalization flow in quantum field theory and statistical physics, Phys. Rept. 363, 223 (2002) [arXiv:hep$\mathrm{ph} / 0005122]$.

[21] Z. Bern, Perturbative quantum gravity and its relation to gauge theory, Living reviews in relativity, www.livingreviews.org/Articles/Volume5/2002-5bern.

[22] Z. Bern, E. Mottola and S. K. Blau, General covariance of the path integral for quantum gravity, Phys. Rev. D 43, 1212 (1991).

[23] Z. Bern, J. Carrasco, D. Forde, H. Ita, and H. Johansson, Unexpected cancellations in gravity theories, [arXiv:0707.1035].

[24] G. de Berredo-Peixoto and I. L. Shapiro, Higher derivative quantum gravity with GaussBonnet term, Phys. Rev. D 71, 064005 (2005) [arXiv:hep-th/0412249].

[25] D. Bettinelli, R. Ferrari and A. Quadri, A comment on the renormalization of the nonlinear sigma model, [arXiv:hep-th/0701197].

[26] N. Bjerrum-Bohr, J. Donoghue and B. Holstein, On the parameterization dependence of the energy momentum tensor and the metric, [arXiv:gr-qc/0610096].

[27] N. Bjerrum-Bohr, J. Donoghue and B. Holstein, Quantum gravitational corrections to the nonrelativistic scattering potential of two masses, Phys. Rev. D 67, 084033 (2003) [Erratum-ibid. D 71, 069903 (2005)] [arXiv:hep-th/0211072].

[28] A. Bonanno and M. Reuter, Proper time flow equation for gravity, JHEP 0502, 035 (2005) [arXiv:hep-th/0410191].

[29] G. Bonneau and F. Delduc, Nonlinear renormalization and the equivalence theorem, Nucl. Phys. B266 (1986) 536.

[30] D. Boulware, Gauge dependence of the effective action, Phys. Rev. D23 (1981) 389.

[31] A. Bovier and G. Felder, Skeleton inequalities and the asymptotic nature of perturbation theory for $\phi^{4}$ theories in two-dimensions and three-dimensions, Commun. Math. Phys. 93, 259 (1984). 
[32] I. Buchbinder, S. Odintsov, I. Shapiro, Effective action in Quantum Gravity, IOP, Bristol, 1992.

[33] C. Burgess, Quantum gravity in everyday life: general relativity as an effective field theory, www. livingreviews.org/Articles/Volume7/2004-burgess.

[34] C. Burgess and G. Kunstatter, On the physical interpretation of the Vilkovisky-DeWitt effctive action, Mod. Phys. Lett. A2 (1987) 875; Erratum A2 (1987) 1003.

[35] P. Breitenlohner, G. Gibbons, and D. Maison, Four-dimensional black holes from KaluzaKlein reduction, Commun. Math. Phys. 120 (1988) 295.

[36] P. Breitenlohner and D. Maison, On nonlinear sigma-models arising in (super-)gravity, Commun. Math. Phys. 209 (2000) 785 [arXiv:gr-qc/9806002].

[37] S. Christensen and M. Duff, Quantum gravity in $2+\epsilon$ dimensions, Phys. Lett. B 79, 213 (1978).

[38] A. Codello and R. Percacci, Fixed points of higher derivative gravity, Phys. Rev. Lett. 97, 221301 (2006) [arXiv:hep-th/0607128].

[39] G. Curci and G. Paffuti, Consistency between the string background field equations of motion and the vanishing of the conformal anomaly, Nucl. Phys. B286 (1987) 399.

[40] B. de Bakker and J. Smit, Gravitational binding in 4D dynamical triangulation, Nucl. Phys. B 484, 476 (1997) [arXiv:hep-lat/9604023].

[41] C. de Calan, P. Faria da Veiga, J. Magnen, and R. Seneor, Constructing the threedimensional Gross-Neveu model with a large number of flavor components, Phys. Rev. Lett. 66 (1991) 3233

[42] S. Deser and P. van Nieuwenhuizen, One loop divergencies of quantized Einstein-Maxwell fields, Phys. Rev. D10 (1974) 401.

[43] B. de Wit, M. Grisaru, H. Nicolai and E. Rabinovici, Two loop finiteness of $d=2$ supergravity, Phys. Lett. 286 (1992) 78 [arXiv:hep-th/9205012].

[44] B. DeWitt, Quantum theory of gravity I: the canonical theory, Phys. Rev. D160 (1967) 1113-1148.

[45] B. DeWitt, Quantum theory of gravity II: the manifestly covariant theory, Phys. Rev. D162 (1967) 1195-1239.

[46] B. DeWitt, Quantum theory of gravity III: applications of the covariant theory, Phys. Rev. D162 (1967) 1239-1262.

[47] J. Dimock, Asymptotic perturbation expansion in the $P_{2}(\phi)$ theory, Commun. Math. Phys. 35 (1974) 347.

[48] B. Dittrich, Partial and complete observables for canonical general relativity, Class. Quant. Grav. 23 (2006) 6155; [arXiv:gr-qc/0507106]. 
[49] W. Dittrich, Bloch-Nordsieck approximation in linearized quantum gravity, in Quantization, Gauge theory, and Strings, Vol. II, A. Semikhatov et al (Eds), World Scientific, 2001.

[50] D. Dou and R. Percacci, The running gravitational couplings, Class. Quant. Grav. 15, 3449 (1998) [arXiv:hep-th/9707239].

[51] M. Fabbrichesi, R. Pettorino, G. Veneziano and G. A. Vilkovisky, Planckian energy scattering and surface terms in the gravitational action, Nucl. Phys. B 419, 147 (1994) [arXiv:hep-th/9309037].

[52] G. Felder, Renormalization group in the local potential approximation, Commun. Math. Phys. 111 (1987) 101.

[53] R. Fernandez, J. Frohlich and A. D. Sokal, Random walks, critical phenomena, and triviality in quantum field theory, Spinger, Berlin etc, 1992.

[54] P. Fischer and D. Litim, Fixed points of quantum gravity in extra dimensions, Phys. Lett. B 638, 497 (2006) [arXiv:hep-th/0602203].

[55] L.H. Ford, Gravitational radiation by quantum systems, Ann. Phys. 144 (1982) 238-248.

[56] E. Fradkin and A. Tseytlin, Higher derivative quantum gravity: one loop counterterms and asymptotic freedom, Nucl. Phys. B 201, 469 (1982).

[57] D. Friedan, Nonlinear models in $2+\epsilon$ dimensions, Ann. Phys. 163 (1985) 318.

[58] S. Fulling, K. Milton, P. Parashar, A. Romeo, K. Shajesh and J. Wagner, How does Casimir energy fall?, [arXiv:hep-th/0702091].

[59] L. J. Garay, Quantum gravity and minimum length, Int. J. Mod. Phys. A 10, 145 (1995) [arXiv:gr-qc/9403008].

[60] R. Gastmans, R. Kallosh and C. Truffin, Quantum gravity near two-dimensions, Nucl. Phys. B 133, 417 (1978).

[61] K. Gawedski and A. Kupainen, Renormalizing the nonrenormalizable, Phys. Rev. Lett 55 (1985) 363.

[62] K. Gawedski and A. Kupainen, Exact renormalization in Gross-Neveu model of quantum fields, Phys. Rev. Lett. 54 (1985) 2191.

[63] K. Gawedzki and A. Kupiainen, Gross-Neveu model through convergent perturbation expansions, Commun. Math. Phys. 102, 1 (1985).

[64] G. Gibbons and S. Hawking, Euclidean quantum gravity, World Scientific, Singapore, 1993.

[65] H. Gies, Renormalizability of gauge theories in extra dimensions, Phys. Rev. D 68, 085015 (2003) [arXiv:hep-th/0305208]. 
[66] J. Gomis and S. Weinberg, Are nonrenormalizable gauge theories renormalizable? Nucl. Phys. B 469, 473 (1996) [arXiv:hep-th/9510087].

[67] M. Goroff and A. Sagnotti, The ultraviolet behavior of Einstein Gravity, Nucl. Phys. B 266, 709 (1986).

[68] M. Green, J. Schwarz, and E. Witten, Superstring theory, Vols 1,2, Cambridge Monographs on Mathematical Physics, 1987.

[69] J. Griffiths, Colliding plane waves in general relativity, Oxford mathematical monographs, Oxford, Clarendon, 1991.

[70] H. Hamber, Discrete and continuum quantum gravity, [arXiv:hep-th/0704.2895].

[71] H. Hamber and R. Williams, Newtonian potential in quantum Regge gravity, Nucl. Phys. B 435, 361 (1995) [arXiv:hep-th/9406163].

[72] S. Hands, A. Kocic, and J. Kogut, Four Fermi theories in fewer than four dimensions, Ann. Phys. 224 (1993) 29-89.

[73] P. Hasenfratz, The theoretical background and properties of perfect actions, Advanced Summer School on Nonperturbative Quantum Field Physics, Peniscola, Spain, 2-6 Jun 1997. [arXiv:hep-lat/9803027].

[74] S. Hawking, Quantum gravity and path integrals, Phys. Rev. D 18, 1747 (1978).

[75] S. Hawking and T. Hertog, Living with ghosts, Phys. Rev D65 (2002) 103515; [arXiv:hepth/0107088].

[76] F. Hofling, C. Nowak and C. Wetterich, Phase transition and critical behaviour of the d $=3$ Gross-Neveu model, Phys. Rev. B 66, 205111 (2002) [arXiv:cond-mat/0203588].

[77] B. Holstein and J. Donoghue, Classical physics and quantum loops, Phys. Rev. Lett. 93, 201602 (2004) [arXiv:hep-th/0405239].

[78] J. Honerkamp, Chiral multi-loops, Nucl. Phys. B 36 (1972) 130.

[79] P. Howe, G. Papadopoulos and K. Stelle, Background field method and the nonlinear $\sigma$-model, Nucl. Phys. B 296 (1988) 26.

[80] D. Kabat and M. Ortiz, Eikonal quantum gravity and Planckian scattering, Nucl. Phys. B 388, 570 (1992) [arXiv:hep-th/9203082].

[81] L. Kadanoff, Statistical Physics: Statics, Dynamics and Renormalization, World Scientific, 2000.

[82] H. Kawai, N. Kawamoto, T. Mogami and Y. Watabiki, Transfer matrix formalism for twodimensional quantum gravity and fractal structures of space-time, Phys. Lett. B 306, 19 (1993) [arXiv:hep-th/9302133]. 
[83] H. Kawai, Y. Kitazawa and M. Ninomiya, Scaling exponents in quantum gravity near two-dimensions, Nucl. Phys. B 393, 280 (1993) [arXiv:hep-th/9206081].

[84] H. Kawai, Y. Kitazawa and M. Ninomiya, Ultraviolet stable fixed point and scaling relations in $(2+\epsilon)$-dimensional quantum gravity, Nucl. Phys. B 404, 684 (1993) [arXiv:hepth/9303123].

[85] H. Kawai and M. Ninomiya, Renormalization group and quantum gravity, Nucl. Phys. B336 (1990) 115-145.

[86] I. Khriplovich and G. Kirilin, Quantum long range interactions in general relativity, J. Exp. Theor. Phys. 981063 (2004) [arXiv: gr-qc/0402018].

[87] C. Klein and O. Richter, Ernst equations, Integrability, and Riemann surfaces, Springer, Berlin etc, 2006.

[88] V. Knizhnik, A. Polyakov and A. Zamolodchikov, Fractal structure of 2d-Quantum Gravity, Mod. Phys. Lett. A 3, 819 (1988).

[89] D. Korotkin and H. Samtleben, Poisson realization and quantization of the Geroch group, Class. Quant. Grav. 14, L151 (1997) [arXiv:gr-qc/9611061].

[90] D. Kreimer, A remark on Quantum Gravity, [arXiv:hep-th/0705.3897].

[91] H. Kröger, Fractal geometry in quantum mechanics, field theory, and spin systems, Phys. Rep. 323 (2000) 81-181.

[92] J. Kubo and M. Nunami, Unrenormalizable theories are predictive, Eur. Phys. J. C 26, 461 (2003) [arXiv:hep-th/0112032].

[93] W. Kummer, On the gauge-independence of the S-matrix, Eur. Phys. J. C 21, 175 (2001) [arXiv:hep-th/0104123].

[94] O. Lauscher and M. Reuter, Ultraviolet fixed point and generalized flow equations of Quantum Gravity, Phys. Rev. D65 (2002) 025013 [arXiv:hep-th/0108040].

[95] O. Lauscher and M. Reuter, Flow equation of quantum Einstein gravity in a higherderivative truncation, Phys. Rev. D 66, 025026 (2002) [arXiv:hep-th/0205062].

[96] O. Lauscher and M. Reuter, Fractal spacetime structure in asymptotically safe gravity, JHEP 0510, 050 (2005) [arXiv:hep-th/0508202].

[97] O. Lauscher and M. Reuter, Asymptotic safety in quantum Einstein gravity: Nonperturbative renormalizability and fractal spacetime structure, in Proceedings Blaubeuren Workshop 2005 on Mathematical and Physical Aspects of Quantum Gravity, [arXiv:hepth/0511260].

[98] D. Litim, Fixed points of quantum gravity, Phys. Rev. Lett. 92, 201301 (2004) [arXiv:hepth/0312114]. 
[99] P. Mazur and E. Mottola, The gravitational measure, solution of the conformal factor problem and stability of the ground state of quantum gravity, Nucl. Phys. B 341, 187 (1990).

[100] A. Mead, Possible connection between gravitation and fundamental length, Phys. Rev. 135 (1964) B849.

[101] C. Misner, Feynman quantization of general relativity, Rev. Mod. Phys. 29 (1957) 497509.

[102] P. Mitter, The exact renormalization group, Contribution to the Encyclopedia of Mathematical Physics, Elsevier 2006 [arXiv:math-ph/0505008].

[103] T. Morris, Elements of the continuous renormalization group, Prog. Theor. Phys. Suppl. 131, 395 (1998) [arXiv:hep-th/9802039].

[104] T. Morris, Renormalizable extra-dimensional models, JHEP 0501, 002 (2005) [arXiv:hep-ph/0410142].

[105] E. Mottola, Functional integration over geometries, J. Math. Phys. 36, 2470 (1995) [arXiv:hep-th/9502109].

[106] M. Niedermaier, unpublished notes, 2005; updated 2007.

[107] M. Niedermaier, Quantized Einstein-Rosen waves, $\operatorname{AdS}(2)$, and spontaneous symmetry breaking, Phys. Lett. B498 (2001) 83; [arXiv:hep-th/0007227].

[108] M. Niedermaier, Renormalization and asymptotic safety in truncated quantum Einstein gravity, JHEP 12 (2002) 066 [arXiv:hep-th/0207143].

[109] M. Niedermaier, Dimensionally reduced gravity theories are asymptotically safe, Nucl. Phys. B673 (2003) 131 [arXiv:hep-th/0304117].

[110] M. Niedermaier and M. Reuter, The Asymptotic Safety Scenario in Quantum Gravity, Living Reviews in Relativity, 9 (2006) 5.

[111] M. Niedermaier and H. Samtleben, An algebraic bootstrap for dimensionally reduced quantum gravity, Nucl. Phys. B 579 (2000) 437 [arXiv:hep-th/9912111].

[112] F. Niedermayer, M. Niedermaier and P. Weisz, "Questionable and unquestionable in the perturbation theory of nonabelian models," Phys. Rev. D 56, 2555 (1997) [arXiv:heplat/9612002].

[113] R. Oehme and W. Zimmermann, Relation between effective couplings for asymptotically free models, Commun. Math. Phys. 97, 569 (1985).

[114] H. Osborn, Renormalization and composite operators in nonlinear $\sigma$-models, Nucl. Phys. B294 (1987) 595.

[115] T. Padmanabhan, Limitations on the operational definition of space-time events and quantum gravity, Class. Quant. Grav. 4, L107 (1987). 
[116] J. Pawlowski, Aspects of the functional renormalisation group, [arXiv:hep-th/0512261].

[117] J. Pawlowski, Geometrical effective action and Wilsonian flows, [arXiv:hep-th/0310018].

[118] P. Peldan, Actions for gravity, with generalizations: a review, Class. Quant. Grav. 11, 1087 (1994) [arXiv:gr-qc/9305011].

[119] R. Percacci and D. Perini, On the ultraviolet behavior of Newton's constant, Class. Quant. Grav. 21, 5035 (2004) [arXiv:hep-th/0401071].

[120] R. Percacci, Further evidence for a gravitational fixed point, Phys. Rev. D 73, 041501 (2006) [arXiv:hep-th/0511177].

[121] R. Percacci and D. Perini, Constraints on matter from asymptotic safety, Phys. Rev. D 67, 081503 (2003) [arXiv:hep-th/0207033].

[122] R. Percacci and D. Perini, Asymptotic safety of gravity coupled to matter, Phys. Rev. D 68, 044018 (2003) [arXiv:hep-th/0304222].

[123] R. Perry and K. Wilson, Perturbative renormalizability with an infinite number of relevant and marginal operators, Nucl. Phys. B 403, 587 (1993).

[124] M. Peskin and D. Schroeder, An Introduction To Quantum Field Theory, HarperCollins Publishers, 1995.

[125] J. Polchinski, String theory, Vols. 1, 2, Cambridge Monographs on Mathematical Physics, 1998.

[126] A. Rebhan, Feynman rules and S-matrix equivalence in the Vilkovisky-deWitt effective action, Nucl. Phys. B 298 (1988) 726.

[127] M. Reuter, Nonperturbative Evolution Equation for Quantum Gravity, Phys. Rev. D 57, 971 (1998) [arXiv:hep-th/9605030].

[128] M. Reuter and F. Saueressig, Renormalization group flow of quantum gravity in the Einstein-Hilbert truncation, Phys. Rev. D 65, 065016 (2002) [arXiv:hep-th/0110054].

[129] S. Robinson, Normalization conventions for Newton's constant and the Planck scale in arbitrary dimensions, [gr-qc/0609060].

[130] J. Romano and C. Torre, Internal time formalism for spacetimes with two Killing vectors, Phys. Rev. D53 (1996); [arXiv:gr-qc/9509055].

[131] L. Rosa, P. Vitale and C. Wetterich, Critical exponents of the Gross-Neveu model from the effective average action, Phys. Rev. Lett. 86, 958 (2001) [arXiv:hep-th/0007093].

[132] C. Rovelli, Quantum gravity, Cambridge Monographs on Mathematical Physics, 2004.

[133] G. Shore, A local renormalization group equation, diffeomorphisms and conformal invariance in sigma models, Nucl. Phys. 286 (1987) 349. 
[134] G. Shore, Superluminality and UV completion, [arXiv:hep-th/0701185].

[135] L. Smolin, A fixed point for quantum gravity, Nucl. Phys. B208 (1982) 439-466.

[136] W. Souma, Non-trivial UV fixed point in quantum gravity, Prog. Theor. Phys. 102 (1999) 181 [arXiv:hep-th/9907027].

[137] W. Souma, Gauge and cutoff function dependence of the ultraviolet fixed point in quantum gravity, [arXiv:gr-qc/0006008].

[138] K. S. Stelle, Renormalization of higher derivative Quantum Gravity, Phys. Rev. D 16, 953 (1977).

[139] K. S. Stelle, Classical Gravity With Higher Derivatives, Gen. Rel. Grav. 9, 353 (1978).

[140] G. 't Hooft and M. Veltman, One loop divergencies in the theory of gravitation, Ann. Inst. Poincaré 20 (1974) 69.

[141] G. 't Hooft, Graviton dominance in ultrahigh-energy scattering, Phys. Lett. B 198, 61 (1987).

[142] C. Teitelboim, The proper time gauge in quantum theory of gravitation, Phys. Rev. D 28, 297 (1983).

[143] C. Teitelboim, Quantum mechanics of the gravitational field in asymptotically flat space, Phys. Rev. D 28, 310 (1983).

[144] E. Tomboulis, Superrenormalizable gauge and gravitational theories, [arXiv:hepth/9702146].

[145] E. Tomboulis, $1 / N$ expansion and renormalization in quantum gravity, Phys. Lett. B70 (1977) 361.

[146] E. Tomboulis, Renormalizability and asymptotic freedom in quantum gravity, Phys. Lett. B97 (1980) 77.

[147] C. Torre, Gravitational observables and local symmetries, Phys. Rev. D 48, 2373 (1993) [arXiv:gr-qc/9306030].

[148] A. Tseytlin, Sigma-model Weyl invariance conditions and string equations of motion, Nucl. Phys. B294 (1987) 383.

[149] B. F. L. Ward, Quantum corrections to Newton's law, Mod. Phys. Lett. A 17, 2371 (2002) [arXiv:hep-ph/0204102].

[150] C. Wetterich, Exact evolution equation for the effective potential, Phys. Lett. B 301, 90 (1993).

[151] C. Wetterich, Effective average action in statistical physics and quantum field theory, Int. J. Mod. Phys. A 16, 1951 (2001) [arXiv:hep-ph/0101178]. 
[152] S. Weinberg, Ultraviolet divergencies in quantum theories of gravitation, in: General relativity, an Einstein Centenary survey, Eds. S. Hawking, W. Israel, Cambridge University Press, 1979.

[153] F. Wilczek, Quantum field theory, Rev. Mod. Phys. 71 (1999) S85; [arXiv:hepth/9803075].

[154] K. Wilson, The renormalization group and critical phenomena, Nobel lecture, 1982.

[155] K. Wilson, Renormalization group and critical phenomena I, II, Phys. Rev. B4 (1971) 3174, B4 (1971) 3184.

[156] K. Wilson, Quantum field theory models in less than 4 dimensions, Phys. Rev D7 (1973) 2911.

[157] A. van de Ven, Two loop quantum gravity, Nucl. Phys. B 378, 309 (1992).

[158] G. Vilkovisky, The Gospel according to deWitt, in: Quantum Theory of Gravity: Eassys in honor of the 60th birthday of B. deWitt, Bristol, 1984.

[159] W. Zimmermann, Reduction in the number of coupling parameters, Commun. Math. Phys. 97, 211 (1985). 